\title{
Chiral algebra, localization, modularity, surface defects, and all that
}

Cite as: J. Math. Phys. 61, 092302 (2020); https://doi.org/10.1063/5.0002661

Submitted: 27 January 2020 . Accepted: 17 August 2020 . Published Online: 11 September 2020

Mykola Dedushenko (D), and Martin Fluder

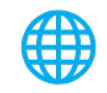

\section{Journal of}

Mathematical Physics

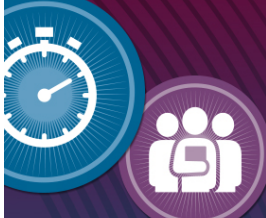

READY TO SUBMIT YOUR RESEARCH?

There are many reasons to publish with us. 


\title{
Chiral algebra, localization, modularity, surface defects, and all that
}

\author{
Cite as: J. Math. Phys. 61, 092302 (2020); doi: 10.1063/5.0002661 \\ Submitted: 27 January 2020 - Accepted: 17 August 2020 • \\ Published Online: 11 September 2020
}

\author{
Mykola Dedushenko ${ }^{1, \text { a) }}$ (D) and Martin Fluder ${ }^{1,2, b)}$
}

\begin{abstract}
AFFILIATIONS
${ }^{1}$ Walter Burke Institute for Theoretical Physics, California Institute of Technology, Pasadena, California 91125, USA

${ }^{2}$ Kavli Institute for the Physics and Mathematics of the Universe (WPI), University of Tokyo, Kashiwa 277-8583, Japan
\end{abstract}

\author{
a) Author to whom correspondence should be addressed: dedushenko@gmail.com \\ b) Electronic mail: fluderm@gmail.com
}

\begin{abstract}
We study the 2D vertex operator algebra (VOA) construction in 4D $\mathscr{N}=2$ superconformal field theories on $S^{3} \times S^{1}$, focusing on both old puzzles and new observations. The VOA lives on a two-torus $\mathbb{T}^{2} \subset S^{3} \times S^{1}$, it is $\frac{1}{2} \mathbb{Z}$-graded, and this torus is equipped with the natural choice of spin structure $(1,0)$ for the $\mathbb{Z}+\frac{1}{2}$-graded operators, corresponding to the NS sector vacuum character. By analyzing the possible refinements of the Schur index that preserves the VOA, we find that it admits discrete deformations, which allows access to the remaining spin structures $(1,1),(0,1)$, and $(0,0)$, of which the latter two involve the inclusion of a particular surface defect. For Lagrangian theories, we perform the detailed analysis: we describe the natural supersymmetric background, perform localization, and derive the gauged symplectic boson action on a torus in any spin structure. In the absence of flavor fugacities, the $2 \mathrm{D}$ and $4 \mathrm{D}$ path integrals precisely match, including the Casimir factors. We further analyze the 2D theory: we identify its integration cycle and the two-point functions and interpret flavor holonomies as screening charges in the VOA. Next, we make some observations about modularity; the $T$-transformation acts on our four partition functions and lifts to a large diffeomorphism on $S^{3} \times S^{1}$. More interestingly, we generalize the four partition functions on the torus to an infinite family labeled by both the spin structure and the integration cycle inside the complexified maximal torus of the gauge group. Members of this family transform into one another under the full modular group, and we confirm the recent observation that the $S$-transform of the Schur index in Lagrangian theories exhibits logarithmic behavior. Finally, we comment on how locally our background reproduces the $\Omega$-background.
\end{abstract}

Published under license by AIP Publishing. https://doi.org/10.1063/5.0002661

\section{INTRODUCTION}

Accessing the strongly coupled and non-perturbative dynamics of a quantum field theory is hard, and even upon incorporating simplifying assumptions, such as supersymmetry or conformal symmetry, techniques allowing for control in such regimes are rare. Certain supersymmetric or protected quantities in supersymmetric field theories nevertheless prove amenable to analytic study, and much of the focus in the last few decades has been on such examples. One prominent class of such theories are four-dimensional $\mathscr{N}=2$ quantum field theories. The infrared (IR) dynamics on the Coulomb branch of such theories has long been understood since the release of the seminal set of works. ${ }^{1-4}$ Incorporating techniques of supersymmetric localization was another major achievement in getting analytical control of such theories. ${ }^{5}$ The advent of a whole new set of structures in these theories also resulted from the invention of the $\Omega$-background. ${ }^{3,4,6-8}$

Recent years have seen another surge of research activity on four-dimensional $\mathscr{N}=2$ theories. More specifically, the discovery and precise formulation of a connection between vertex operator algebras (VOAs) and four-dimensional superconformal field theories (SCFTs) in Ref. 9 has sparked a rekindled research interest (see, e.g., Refs. 10-45 for subsequent developments). Such VOAs are rich yet rigid objects that, quite surprisingly, allow us to gain a non-perturbative access to an algebraically closed sector in the operator product expansion (OPE) data of the superconformal field theory. The latter fact is completely independent of whether the theory admits a weakly coupled Lagrangian description or not. This has grown into a subfield by itself, sometimes referred to as the "SCFT/VOA correspondence." 
In this work, we are set to contribute to this subfield by combining the latest developments for the (older) Lagrangian technique of supersymmetric localization with the newer ideas on four-dimensional $\mathscr{N}=2$ superconformal field theories. The original construction of Ref. 9 was formulated in the flat Euclidean space $\mathbb{R}^{4}$, though the need to also understand it in other backgrounds was repeatedly raised over the years. Here, we study the chiral algebra construction ${ }^{46}$ on the $S^{3} \times S^{1}$ geometry, which, incidentally, is also the geometry relevant for studying the superconformal index, including its Schur limit. ${ }^{47,48}$ Given that the VOA precisely captures the so-called Schur sector of the theory (i.e., states counted by the Schur index), we take it as no coincidence, but rather as an indication that the $S^{3} \times S^{1}$ background is in many ways an inherently natural playground for the study of SCFT/VOA correspondence.

As a courtesy to busy readers, and to avoid getting lost in technicalities, we now provide a somewhat more detailed overview of the main results presented in this work.

\section{A. Chiral algebra on $S^{3} \times S^{1}$}

The original construction ${ }^{9}$ of the two-dimensional vertex operator algebra as a subsector of the full four-dimensional $\mathscr{N}=2$ superconformal field theory relies on a powerful "abstract" operator approach to the four-dimensional theory, which does not require a Lagrangian description. From such a point of view, any conformal theory can be placed on $S^{3} \times \mathbb{R}$ via a Weyl transformation (on Weyl invariance of conformal theories, see, e.g., Ref. 49). If we further close $\mathbb{R}$ to a circle, which we label as $S_{y}^{1}$ throughout this paper, we find that the theory breaks supersymmetry unless we define it in a twisted sector with respect to the R-symmetry. In this case, one manages to preserve half of the flat space supercharges, namely, those commuting with $E-R$, where $E$ is the dilatation generator (that generates translations along $\mathbb{R}$ in the $S^{3} \times \mathbb{R}$ geometry) and $R$ is a Cartan generator of the $S U(2)_{R}$ symmetry. The surviving supercharges, together with isometries of $S^{3}$ and the $U(1)_{R} \times U(1)_{r} \subset S U(2)_{R} \times U(1)_{r}$ R-symmetry subgroup, form a supersymmetry algebra,

$$
\mathfrak{s u}(2 \mid 1)_{\ell} \oplus \mathfrak{s u}(2 \mid 1)_{r},
$$

which is centrally extended by $E-R$. This should be regarded as an $\mathscr{N}=2$ superconformal symmetry on $S^{3} \times S^{1}$. Conformal invariance is crucial as this algebra contains $U(1)_{r}$ that is only known to be unbroken in conformal theories. A similar algebra without central extension has previously appeared as three-dimensional $\mathscr{N}=4$ supersymmetry on $S^{3}$ in Ref. 50 . In this case, the algebra admitted central extensions due to the addition of masses and Fayet-Iliopoulos (FI) parameters in Lagrangian theories. In the present context, this $E-R$ should be regarded as a mass-like central charge. One can also introduce additional mass-like central charges by turning on flavor symmetry holonomies around the $S_{y}^{1}$ circle (corresponding to flavor fugacities in the index), and they can be regarded as lifts of the three-dimensional mass terms. However, there is no interesting lift of three-dimensional FI parameters because we study four-dimensional superconformal field theories, and even if they admit Lagrangian descriptions, they do not have any Abelian factors in their gauge groups.

The algebra (1) contains everything required to define the two-dimensional VOA sector. All ingredients of the original construction from Ref. 9 can be easily translated to this context, and one finds that the VOA is now supported on the torus $S_{\varphi}^{1} \times S_{y}^{1}$, where $S_{\varphi}^{1} \subset S^{3}$ is a great circle. The labeling of circles stems from the coordinates we use; $S_{y}^{1}$ is parameterized by $y \in[0, \beta \ell]$, while $S^{3}$ is parameterized, just like in Ref. 50 , by the fibration coordinates $(\theta, \varphi, \tau)$ in which $S_{\varphi}^{1}$ is a circle parameterized by $\varphi$ that sits at $\theta=\pi / 2$, where the $\tau$-circle shrinks to a point (see Sec. II B for details). Since we essentially view $S^{3}$ as $D^{2} \times S_{\tau}^{1}$ with $S_{\tau}^{1}$ shrinking at $\partial D^{2}=S_{\varphi}^{1}$, we sometimes refer to $S_{\varphi}^{1}$ as the "boundary," even though $S^{3}$ is, of course, closed.

\section{B. Localization on $S^{3} \times S^{1}$}

With the exception of the above generalities, in this paper, we focus on Lagrangian $\mathscr{N}=2$ superconformal field theories in four dimensions. In this case, by employing supersymmetric localization on a rigid background of the form $S^{3} \times S_{y}^{1}$, we explicitly localize a given Lagrangian superconformal field theory and find that it indeed reproduces the expected two-dimensional VOA on the torus $S_{\varphi}^{1} \times S_{y}^{1} \subset S^{3} \times S_{y}^{1}$, described as a gauged symplectic boson. The symplectic boson VOA is also known as a $\beta-\gamma$ system of weight $\frac{1}{2}$, and we interchangeably use the two terms.

To derive the two-dimensional VOA on the torus, we first define the appropriate rigid supersymmetric $S^{3} \times S_{y}^{1}$ background, reproducing the superconformal index. In doing so, we analyze the supersymmetry algebra and classify the possible fugacities and their preserved subalgebras. In order to retain the VOA construction, the minimal amount of supersymmetry we ought to preserve is $\mathfrak{s u}(1 \mid 1)_{\ell} \oplus \mathfrak{s u}(1 \mid 1)_{r}$. We find that we may turn on fugacities preserving an $\mathfrak{s u}(1 \mid 1)_{\ell} \oplus \mathfrak{s u}(2 \mid 1)_{r}$ subalgebra (which can be further broken to the minimal one by defects). Surprisingly, this goes beyond the fugacity in the Schur limit, which is well-known to be relevant for the chiral algebra construction. Specifically, we are allowed to turn on discrete fugacities $M, N \in \mathbb{Z}$, where $N$ corresponds to an insertion of $e^{2 \pi i N(R+r)}$ in the Schur index, while non-zero $M$ modifies the geometry. For non-zero $M$, the $S^{3} \times S_{y}^{1}$ is no longer equipped with a product metric, but rather one rotates $S^{3}$ by $\Delta \varphi=\Delta \tau=2 \pi M$ as we go around the $S_{y}^{1}$. As we shall argue, these deformations do not affect the VOA construction but change the complex structure of the torus and affect the boundary conditions (spin structure) upon going around one of the cycles, $S_{y}^{1}$ (see below). The inclusion of $M$ actually does not affect the partition function, but throughout we keep both $M$ and $N$ as generic integers.

We perform localization for the $\mathscr{N}=2$ vector multiplets; in this case, the two-dimensional theory is determined indirectly. As we show, the Yang-Mills action is Q-exact, and thus, the four-dimensional theory solely localizes to a one-loop determinant piece. Nonetheless, the two-dimensional action can be "bootstrapped" from the knowledge of the partition function and the four-dimensional propagators and is given by the small $b c$ ghost system on the torus. 
Localization for the four-dimensional $\mathscr{N}=2$ hypermultiplets more straightforwardly reproduces the two-dimensional theory. Specifically, the remnant "classical" piece in the localization precisely reduces to the two-dimensional symplectic boson theory on the boundary torus $S_{\varphi}^{1} \times S_{y}^{1} \subset S^{3} \times S_{y}^{1}$. As alluded above, the discrete label $M$ changes the complex structure of the torus (essentially, $M \neq 0$ differs from $M=0$ by precisely $M$ Dehn twists or a $T^{M}$ modular transformation), while $N$ changes the spin structure (periodicity) of symplectic bosons along $S_{y}^{1}$.

The localization from four to two dimensions produces almost no overall one-loop determinant, except for a simple Casimir energy factor. The part of it that depends on the gauge holonomy around the $S_{y}^{1}$ cancels between the hypermultiplets and vector multiplets as a consequence of conformal invariance. If we turn on flavor holonomies, however, there is a simple leftover term that survives and describes the mismatch of the four- and two-dimensional path integrals. It is given by

$$
q^{-\frac{1}{4} \sum_{w_{f} \in \mathbf{R}_{f}}\left\langle w_{f}, a_{f}\right\rangle^{2}}
$$

where the sum goes over all weights in a flavor symmetry representation $\mathbf{R}_{f}$ in which the matter transforms, $a_{f}$ is the background holonomy in the Cartan of flavor group, and $q=e^{2 \pi i \tau}$, where $\tau$ is a complex structure of the torus. This formula can also be generalized to non-Lagrangian theories relying on the results of Ref. 13, but this goes beyond the scope of this paper.

\section{Spin structures}

All operators in the VOA have either integral or half-integral conformal dimensions, as a consequence of the SCFT/VOA correspondence for general, not necessarily Lagrangian SCFTs. Thus, in order to place the VOA on a torus, one has to pick a particular spin structure, which amounts to choosing operators of half-integral conformal degree to be either periodic or anti-periodic along the one-cycles of $\mathbb{T}^{2}$. [This can be interpreted as a background connection for the $\mathbb{Z}_{2}$ automorphism acting as $(-1)^{2 L_{0}}$, which always makes sense in the general context of SCFT/VOA correspondence but can act trivially if all eigenvalues of $L_{0}$ are integers.] The canonical choice that follows from how we put a four-dimensional theory on $S^{3} \times S_{y}^{1}$ is $(1,0)$, meaning that spinors are anti-periodic (in the NS sector) along $S_{\varphi}^{1}$ and periodic (in the R sector) along $S_{y}^{1}$.

We mentioned above that the nontrivial $N \bmod 2$ amounts to flipping the spin structure of symplectic bosons along $S_{y}^{1}$. This is, in fact, more general and holds in an arbitrary VOA (arising from a superconformal field theory): starting with the "standard" NS sector vacuum character, corresponding to $(1,0)$ spin structure, and turning on $N \bmod 2$ in the four-dimensional background, we arrive at the torus partition function with $(1,1)$ spin structure. Given our general localization, we immediately get the corresponding four-dimensional result, $Z^{(1,1)}$, by setting $N=1$, which, incidentally, is the "modified Schur index" considered by Razamat. ${ }^{51}$ Of course, it precisely agrees with the NS-NS character. (Since we only work with chiral algebras, we sometimes use the NS-NS and NS-R terminology to denote spin structures along the pair of one-cycles of $\mathbb{T}^{2}$, which should not be confused with the spin structures of left and right movers along the same $S^{1}$, which is a widely used convention in string theory.)

In two dimensions, we can also change the periodicity of the fields along the other, here $S_{\varphi}^{1}$, cycle to obtain the remaining $(0,1)$ and $(0,0)$ spin structures. However, because the $S_{\varphi}^{1}$-cycle is contractible in $S^{3}$, we cannot continuously change the periodicity of the four-dimensional fields along it; thus, we have to make $S_{\varphi}^{1}$ non-contractible. To do so in a physically sensible way, we have to introduce a surface defect at $\theta=0$, i.e., describing $S^{3}$ as a circle fibration over the disk $D^{2}, \theta=0$ is at the origin of $D^{2}$, and the defect extends in the $\tau$ and $y$ directions.

\section{The R-symmetry defect}

The surface defect that can switch the $S_{\varphi}^{1}$ spin structure should act similarly to the $e^{2 \pi \mathrm{i}(R+r)}$ monodromy that was able to alter the $S_{y}^{1}$ spin structure. (Note that $e^{2 \pi \mathrm{i}(R+r)}= \pm 1$, since $R+r \in \frac{1}{2} \mathbb{Z}$ in our conventions.) Specifically, we first define the symmetry interface that does the transformation $e^{2 \pi \mathrm{i}(R+r)}$ on a theory-we call it the canonical R-symmetry interface. Then, we claim that the relevant surface defect should sit at the boundary of such an interface. If we place the surface defect at $\theta=0$, with the canonical R-symmetry interface ending on it, we get what we want: operators that have half-integral dimension in the VOA will obtain the opposite periodicity around $S_{\varphi}^{1}$. In this way, we can achieve the $(0,1)$ and $(0,0)$ or NS-R and R-R spin structures from four dimensions.

Such surface defects should exist in general superconformal field theories, but they are by no means unique. In fact, they correspond to the Ramond sector modules of the VOA, and there might be many of them. For Lagrangian theories, we define in the main text and in Appendix D the simplest possible pair of such defects. Because $e^{2 \pi i(R+r)}$ acts trivially on the vector multiplets, we define defects that only directly couple to hypermultiplets by changing their asymptotic behavior in the vicinity of the defect. This is closely related to the monodromy defect considered in Ref. 23, and we elaborate more on their relation in Sec. II F. We further generalize the localization answer to include this defect at $\theta=0$. In this way, we end up with four partition functions, $Z^{(1,0)}, Z^{(1,1)}, Z^{(0,1)}, Z^{(0,0)}$, labeled by the spin structure, where the first one is the usual Schur index and the second one is, of course, the "modified Schur index" of Razamat.

\section{E. The two-dimensional theory}

Once we have the two-dimensional action of gauged symplectic bosons, it is straightforward to study its various properties, such as the proper integration cycle and the two-point functions in all spin structures. One interesting observation that we make is that in the presence 
of flavor holonomies-which appear as mass-like central charges in the supersymmetry algebra-vertex operators charged under the flavor symmetries fail to remain holomorphic. The sector that remains holomorphic is formed by flavor-neutral operators. At the level of the VOA, it corresponds to the well-known operation of screening. Somewhat formally, it expands the class of vertex algebras we might study. One simple example we describe is a free hypermultiplet: turning on its $U(1)_{F} \subset S U(2)_{F}$ flavor holonomy screens the free symplectic boson VOA to a slightly simpler VOA described by a pair of chiral bosons.

\section{F. Modularity of the Schur index}

It has long been suggested that the four-dimensional Schur index observes modular properties (see, for example, Refs. 51 and 52 ). ${ }^{53}$ Naively, one expects that the modular properties are related to an exchange of the "thermal cycle" with the Hopf fiber of the index. Of course, with the advent of Ref. 9, the identification of the Schur index with some vacuum character of a two-dimensional chiral algebra immediately suggests that the Schur index ought to be part of a modular vector. Indeed, in Ref. 26, differential equations observing modular properties were derived, and it was suggested that the vacuum character-or the Schur index-is a solution of them.

Nevertheless, a four-dimensional understanding of the modular properties of the two-dimensional character remains an unresolved problem. For Lagrangian theories, our discussion of the spin structures of the torus partition function and its relation to the four-dimensional Schur index gives us immediately the $T$-modular transformation of the latter. In particular, we explicitly find the following general result:

$$
\begin{array}{ll}
Z^{(1,0)}\left(\tau+1, a_{f}\right) \propto Z^{(1,1)}\left(\tau, a_{f}\right), & Z^{(1,1)}\left(\tau+1, a_{f}\right) \propto Z^{(1,0)}\left(\tau, a_{f}\right), \\
Z^{(0,0)}\left(\tau+1, a_{f}\right) \propto Z^{(0,0)}\left(\tau, a_{f}\right), & Z^{(0,1)}\left(\tau+1, a_{f}\right) \propto Z^{(0,1)}\left(\tau, a_{f}\right) .
\end{array}
$$

In order to shed light on the action of the modular $S$-transformation, we introduce novel (formal) partition functions labeled by two additional indices, $m, n \in \mathbb{Z}$ and $Z_{(m, n)}^{\left(v_{1}, v_{2}\right)}$. They are defined as the partition function in the given spin structure $\left(v_{1}, v_{2}\right)$, but now with the modified contour $\mathbb{T}_{(m, n)}$ of the holonomy integral in the localization formula, labeled by the integers $m$ and $n$. The upshot of introducing this extended, infinite set of partition functions is that they exhibit a simple behavior under modular transformations. ${ }^{53}$ For instance, we find that

$$
\begin{aligned}
& Z_{(m, n)}^{\left(v_{1}, v_{2}\right)}\left(-\frac{1}{\tau}, \frac{a_{f}}{\tau}\right) \propto Z_{(-n, m)}^{\left(v_{2}, v_{1}\right)}\left(\tau, a_{f}\right), \\
& Z_{(m, n)}^{\left(v_{1}, v_{2}\right)}\left(\tau+1, a_{f}\right) \propto Z_{(m+n, n)}^{\left(v_{1}, v_{2}+v_{1}\right)}\left(\tau, a_{f}\right) .
\end{aligned}
$$

Thus, we suggest that the objects $Z_{(m, n)}^{\left(v_{1}, v_{2}\right)}$ furnish an (infinite-dimensional projective) representation of $S L(2, \mathbb{Z})$, and given the relation to twodimensional chiral algebras, we expect it to truncate, i.e., there are relations among them, and a-possibly finite-set of them transform as some modular vector. ${ }^{26}$ Physically, the remanent independent objects $Z_{(m, n)}^{\left(v_{1}, v_{2}\right)}$ are expected to correspond to partition functions in the presence of general defects (corresponding to the non-trivial modules of the two-dimensional chiral algebra, 18,23,54).

Finally, we discuss two simple examples and explicitly observe that the $S$-transformation of the Schur index exhibits logarithmic behavior, which is in accordance with the expected solutions to the modular differential equations derived in Ref. 26.

\section{G. Relation to the (flat) $\Omega$-background $\mathbb{R}_{\varepsilon}^{2} \oplus \mathbb{R}^{2}$}

For a while, it has been suggested ${ }^{55}$ (see also Refs. 40 and 56) that the two-dimensional chiral algebra can be obtained by an $\Omega$-deformation ${ }^{3,4}$ of the holomorphic-topological twist, introduced by Kapustin in Ref. 57 (see Refs. 44 and 45 for recent results to this end). Indeed, our analysis of the $S^{3} \times S^{1}$ background, with the two-dimensional theory arising on the torus, $\mathbb{T}^{2} \subset S^{3} \times S^{1}$, suggests that the (flat space) $\Omega$-background is hidden as some "local" (and decompactified) version near the torus. ${ }^{58}$ In Sec. VI, we comment on this connection in four dimensions, determining an expansion of the $S^{3} \times S^{1}$ background in the vicinity of the torus and thereby explicitly obtaining the flat $\Omega$-background $\mathbb{R}_{\varepsilon}^{2} \oplus \mathbb{R}^{2}$. The theory then effectively localizes to the tip of the $\Omega$-background, $\mathbb{R}_{\varepsilon}^{2}$, giving the symplectic boson action in the remaining (flat) two-dimensional space. We remark here that our background seems to be in accordance with the recent results in Refs. 44 and 45.

The remainder of this paper is organized as follows: We start in Sec. II by setting the stage and define the supersymmetric background on $S^{3} \times S^{1}$. We further discuss the possible fugacities including their preserved subalgebra and argue that we may slightly deform away from the Schur limit while still preserving the VOA construction. Furthermore, we introduce the notion of spin structures on the torus to the game and discuss how they are realized from a four-dimensional point of view, which marks the inception of the canonical R-symmetry interface and surface defect. In Sec. III, we explicitly localize the $S^{3} \times S^{1}$ partition function onto the gauged symplectic bosons in two dimensions. This includes the results for the (four) different spin structures and the R-symmetry surface defect. We point out various subtleties along the way. In Sec. IV, we consequently discuss the various aspects of the resulting two-dimensional theory. This includes the determination of its integration cycle, the propagators, and the interpretation of flavor fugacities as screening charges in two dimensions. In Sec. $V$, we then discuss some implications of our results toward understanding the modular properties of the Schur index. Finally, in Sec. VI, we mention the 
connection to the flat $\Omega$-background. We close in Sec. VII with a brief discussion and mention some work for the future. We collect some more technical details in Appendixes A-E.

\section{THE INDEX, THE BACKGROUND, AND THE ALGEBRA}

In this section, we provide the necessary details of the constructions explored in the remainder of this paper. We start by recalling the definition of the superconformal index of four-dimensional $\mathscr{N}=2$ superconformal field theories and choose a particular representation of it convenient for our purposes. We then proceed to describe the rigid background in four-dimensional $\mathscr{N}=2$ conformal supergravity, which is appropriate for studying the index. Such a background, of course, ought to have the topology of $S^{3} \times S_{y}^{1}$ (we denote the Euclidean time direction by $y$ ). However, we may also replace $S_{y}^{1}$ by an interval or $\mathbb{R}$. In the latter case, it can be obtained by a Weyl transformation from flat space.

We further explicitly describe the most general fugacities in the superconformal index compatible with the supercharge $\mathscr{Q}^{H}$, which is required to be preserved for the vertex operator algebra (VOA) construction, and explain in detail how the VOA construction works on $S^{3} \times S_{y}^{1}$. In particular, we obtain a VOA on the torus $\mathbb{T}^{2} \subset S^{3} \times S_{y}^{1}$. Interestingly, such a specialization of fugacities goes slightly beyond the well-known Schur limit of the superconformal index. Specifically, we add a discrete parameter that switches the spin structure of the torus (along $S_{y}^{1}$ ) at the level of the VOA. Furthermore, we introduce a surface defect that switches the spin structure along the other cycle of the torus-such defects correspond to the Ramond sector modules for the VOA.

\section{A. Different representations of the superconformal index}

The superconformal index in four-dimensional $\mathscr{N}=2$ superconformal field theories was introduced in Refs. 59-61. In its most basic form, and given a choice of the supercharge $\mathscr{Q}$, it is defined as

$$
\mathscr{I}\left(\mu_{i}\right)=\operatorname{Tr}_{\mathscr{H}_{S^{3}}}(-1)^{F} e^{-\mu_{i} T_{i}} e^{-L \delta}
$$

where the trace is taken over the Hilbert space of radial quantization $\mathscr{H}_{S^{3}}$ and $F$ is the fermion number, and we introduced the definition

$$
\delta \equiv 2\left\{\mathscr{Q}, \mathscr{Q}^{\dagger}\right\}
$$

Furthermore, $T_{i}$ is a maximal set of mutually commuting generators that necessarily also commute with $\mathscr{Q}$. Mirroring the choice in Ref. 48 , we pick the particular supercharge $\mathscr{Q}=\widetilde{\mathscr{Q}}_{1 \text { - }}$ to define the index. Thus, we fix

$$
\delta=\widetilde{\delta}_{1-}=E-2 j_{2}-2 R+r
$$

and the maximal set of commuting generators consists of the following anti-commutators:

$$
\begin{aligned}
& \delta_{1-}=E-2 j_{1}-2 R-r, \\
& \delta_{1+}=E+2 j_{1}-2 R-r, \\
& \widetilde{\delta}_{2+}=E+2 j_{2}+2 R+r .
\end{aligned}
$$

A key property of the superconformal index is its independence of $L$. This is due to the pairwise cancellations of non-zero modes of $\widetilde{\delta}_{1}$. Thus, we may shift $L$, as long as we are preserving the convergence of (5). In particular, shifting $L$ by various linear combinations of chemical potentials $\mu_{i}$ is equivalent to redefining $T_{i} \rightarrow T_{i}+c \delta$, where $c$ is some number. Because such redefinitions do not affect the answer, we may write the following equivalent formulas for the index:

$$
\begin{aligned}
& \mathscr{I}(\rho, \sigma, \tau)=\operatorname{Tr}(-1)^{F} \rho^{\frac{1}{2}\left(E-2 j_{1}-2 R-r\right)} \sigma^{\frac{1}{2}\left(E+2 j_{1}-2 R-r\right)} \tau^{\frac{1}{2} \widetilde{\delta}_{2+}} e^{-L \widetilde{\delta}_{1-}}, \\
& \mathscr{I}(p, q, t)=\operatorname{Tr}(-1)^{F} p^{\frac{1}{2}\left(E+2 j_{1}-2 R-r\right)} q^{\frac{1}{2}\left(E-2 j_{1}-2 R-r\right)} t^{R+r} e^{-L \widetilde{\delta}_{1-}}, \\
& \mathscr{I}(p, q, t)=\operatorname{Tr}(-1)^{F} p^{j_{2}+j_{1}-r} q^{j_{2}-j_{1}-r} t^{R+r} e^{-L \widetilde{\delta}_{1-}} .
\end{aligned}
$$

Passing from (11) to (12) involves the change of variables $p=\tau \sigma, q=\tau \rho$, and $t=\tau^{2}$ and a shift of $L$, while passing from (12) to (13) is accomplished solely by shifting $L$.

Note that even though the answer is $L$-independent, $L$ also plays the role of a regulator, regularizing the trace over an infinite-dimensional Hilbert space. The importance of this factor differs in the alternative representations of the index. For example, since the factor $(\rho \sigma \tau)^{\frac{1}{2} E}$ ensures 
convergence, as long as $|\rho \sigma \tau|<1$ (with some extra assumptions), we may safely put $L=0$ in (11). Similarly, we can set $L=0$ in (12) as long as $|p q|<1$. However, we cannot put $L=0$ in (13), as none of the other factors can obviously serve as a regulator.

In the following, we will set $L=0$ and work with the representation of the superconformal index given in (12). We further perform the following change of variables:

$$
\begin{aligned}
(p q)^{1 / 2} & =e^{-\beta}, \\
\frac{p}{q} & =e^{2 \mathrm{i} \beta \zeta}, \\
\frac{t}{(p q)^{1 / 2}} & =e^{\mathrm{i} \beta \gamma}
\end{aligned}
$$

upon which the index takes the following form:

$$
\mathscr{I}(\beta, \zeta, \gamma)=\operatorname{Tr}_{\mathscr{H}} S_{S^{3}}(-1)^{F} e^{-\beta E} e^{\beta R} e^{2 \mathrm{i} \beta \zeta j_{1}} e^{\mathrm{i} \beta \gamma(R+r)}
$$

Indeed, this representation is the most convenient for the path integral interpretation. We put the theory on a space $S^{3}$, and the factor $e^{-\beta E}$ suggests that we have to evolve it for the Euclidean time $\beta$. The additional factors $e^{\beta R} e^{2 \mathrm{i} \beta \zeta j_{1}} e^{\mathrm{i} \beta \gamma(R+r)}$ further imply that afterward we have to perform various symmetry transformations on the system, and the trace means that we close the time direction into a circle $S_{y}^{1}$. Finally, $(-1)^{F}$ implies that fermions ought to have periodic boundary conditions (up to the twists introduced by $e^{\beta R} e^{2 \mathrm{i} \beta \zeta j_{1}} e^{\mathrm{i} \beta \gamma(R+r)}$ ).

Thus, we have to put the theory on a Euclidean space given by $S^{3} \times S_{y}^{1}$, defined in a twisted sector, where upon going once around $S_{y}^{1}$, we perform the R-symmetry transformations $e^{\beta R} e^{\mathrm{i} \beta \gamma(R+r)}$ and rotate $S^{3}$ by $e^{2 \mathrm{i} \beta \zeta j_{1}}$. The latter means that the geometry can be constructed by first taking $S^{3} \times I$ with the product metric, where $I$ is an interval, and then identifying the two boundary three-spheres by a rotation generated by $e^{2 i \beta \zeta j_{1}}$. It is straightforward to describe a background satisfying these criteria, and we do so in Subsection II B. Quite importantly, this background should preserve both $U(1)_{R} \subset S U(2)_{R}$ and $U(1)_{r}$ R-symmetries, simply because we work in a twisted sector with respect to both $R$ and $R+r$.

On the contrary, the background does not have to preserve the supercharge $\widetilde{\mathscr{Q}}_{1-}$ used in the original definition of the index (5). Once we have arrived at expression (17) that concerns a particular way to count states in the radial quantization Hilbert space $\mathscr{H}_{S^{3}}$, it no longer matters what supercharge we started with in (5). In what follows, we will investigate what supersymmetry is preserved by such a background for various values of the fugacities in (17).

\section{B. The background}

Using off-shell supergravity to construct rigid supersymmetric backgrounds has become a standard technique in supersymmetry literature. $^{63}$ (This technique goes back to as early as Refs. 151-153 in the context of topologically twisted theories. Of course, it is also possible to define (rigid) supersymmetry on the $S^{3} \times \mathbb{R}$ background using other methods. ${ }^{154}$ Nevertheless, we are going to follow the general approach of Ref. 62.) The relevant supergravity theory for our problem is given by the four-dimensional $\mathscr{N}=2$ off-shell conformal supergravity of Refs. 63-65 based on the standard Weyl multiplet. (General rigid supersymmetric backgrounds have been analyzed in Ref. 155 within the setting of four-dimensional conformal supergravity.) It has been successfully used to put general four-dimensional $\mathscr{N}=2$ gauge theories on the ellipsoid in Refs. 66 and 67 in which case the theory does not have to be conformal. We follow the conventions of Ref. 67 (see also Appendix A). As we will see, theories that are not conformal quantum-mechanically appear to break supersymmetry in our $S^{3} \times S^{1}$ background, and therefore, for the scope of this paper, we focus solely on conformal theories. ${ }^{68}$

We parameterize the space $S^{3} \times S_{y}^{1}$ by variables $(\theta, \varphi, \tau, y)$, where $\theta \in[0, \pi / 2], \varphi \in[-\pi, \pi]$, and $\tau \in[0,2 \pi]$ are "fibration" coordinates for the three-sphere $S^{3}$ of radius $\ell$ and $y \in[0, \beta \ell]$ parameterizes the circle $S_{y}^{1}$ of circumference $\beta \ell$. The corresponding metric then reads

$$
\mathrm{d} s^{2}=\mathrm{d} y^{2}+\ell^{2}\left[\mathrm{~d} \theta^{2}+\sin ^{2} \theta\left(\mathrm{d} \varphi+\frac{\zeta}{\ell} \mathrm{d} y\right)^{2}+\cos ^{2} \theta\left(\mathrm{d} \tau+\frac{\zeta}{\ell} \mathrm{d} y\right)^{2}\right]
$$

where we further introduced the additional deformation $\zeta$, which is related to the "standard" fugacities $p, q$, and $t$ via equations (14)-(16). Note that for $\zeta=0$, this is just a product of a round $S^{3}$ with $S_{y}^{1}$, while non-zero $\zeta$ introduces a twist, i.e., as we go around the $S_{y}^{1}$, the threesphere is rotated by $\Delta \varphi=\Delta \tau=\beta \zeta$. Alternatively, we could write our metric in terms of the coordinates $\widetilde{\varphi}=\varphi+\zeta y / \ell$ and $\widetilde{\tau}=\tau+\zeta y / \ell$ in which it would simply become a product metric, where gluing a patch $y \in(0, \beta \ell)$ into a circle, i.e., identifying $y=0$ with $y=\beta \ell$, would involve a rotation ("twist") by $\Delta \varphi=\Delta \tau=\beta \zeta$. This rotation is precisely generated by $j_{1}$, which appears in the definition of the superconformal index in equations (11)-(13). We prefer to use the coordinates $(\theta, \varphi, \tau, y)$, in which the metric takes the form (18), and up to the R-symmetry twists 
introduced in Subsection II A, all the variables of the theory are periodic in the $y$ direction. We conveniently work with the following vierbein:

$$
\begin{array}{rlrl}
e^{1} & =\ell \mathrm{d} \theta, & e^{2} & =\ell \sin \theta\left(\mathrm{d} \varphi+\frac{\zeta}{\ell} \mathrm{d} y\right), \\
e^{4}=\mathrm{d} y, & e^{3}=\ell \cos \theta\left(\mathrm{d} \tau+\frac{\zeta}{\ell} \mathrm{d} y\right),
\end{array}
$$

in which the spin connection has the following non-vanishing components:

$$
\begin{array}{ll}
\Omega_{\varphi}^{21}=-\Omega_{\varphi}^{12}=\cos \theta, & \Omega_{\tau}^{13}=-\Omega_{\tau}^{31}=\sin \theta, \\
\Omega_{y}^{13}=-\Omega_{y}^{31}=\frac{\zeta}{\ell} \sin \theta, & \Omega_{y}^{21}=-\Omega_{y}^{12}=\frac{\zeta}{\ell} \cos \theta .
\end{array}
$$

We define rigid supersymmetry for four-dimensional $\mathscr{N}=2$ superconformal field theories by coupling to the $\mathscr{N}=2$ standard Weyl supergravity multiplet (in the rigid limit). Its component fields are

$$
\left(g_{\mu v},\left(V_{\mu}\right)_{B}^{A}, \widetilde{V}_{\mu}, \bar{T}_{\mu v}, T_{\mu v}, M, \psi_{\mu A}, \bar{\psi}_{\mu A}, \eta_{A}, \bar{\eta}_{A}\right)
$$

where $g_{\mu \nu}$ is the metric, $\left(V_{\mu}\right)_{B}^{A}$ is a gauge field for the $S U(2)_{R}$ symmetry, $\widetilde{V}_{\mu}$ is a gauge field for the $U(1)_{r}$ symmetry, $\bar{T}_{\mu \nu}\left(T_{\mu \nu}\right)$ is an $($ anti- $)$ self-dual tensor, $M$ is a scalar, $\psi_{\mu A}\left(\bar{\psi}_{\mu A}\right)$ is an (anti-)chiral gravitino, and finally $\eta_{A}\left(\bar{\eta}_{A}\right)$ is an (anti-)chiral spinor. Then, supersymmetry is determined by nontrivial chiral spinors $\xi_{\alpha A}$ and anti-chiral spinors $\bar{\xi}_{A}^{\dot{\alpha}}$, where $A=1,2$ is the $S U(2)_{R}$ index, which together with appropriate background supergravity fields allow for the vanishing of the supersymmetry variations of the fermions $\bar{\psi}_{\mu A}, \psi_{\mu A}, \bar{\eta}_{A}$, and $\eta_{A}$. This implies that we ought to solve the following system of conformal Killing spinor equations (see Appendix A for relevant notations):

$$
\begin{aligned}
\mathscr{D}_{\mu} \xi_{A}+T^{v \rho} \sigma_{\nu \rho} \sigma_{\mu} \bar{\xi}_{A} & =-\mathrm{i} \sigma_{\mu} \bar{\xi}_{A}^{\prime}, \\
\mathscr{D}_{\mu} \bar{\xi}_{A}+\bar{T}^{v \rho} \bar{\sigma}_{\nu \rho} \bar{\sigma}_{\mu} \xi_{A} & =-\mathrm{i} \bar{\sigma}_{\mu} \xi_{A}^{\prime}, \\
\sigma^{\mu} \bar{\sigma}^{v} \mathscr{D}_{\mu} \mathscr{D}_{\nu} \xi_{A}+4 \mathscr{D}_{\rho} T_{\mu \nu} \sigma^{\mu v} \sigma^{\rho} \bar{\xi}_{A} & =M \xi_{A}, \\
\bar{\sigma}^{\mu} \sigma^{v} \mathscr{D}_{\mu} \mathscr{D}_{\nu} \bar{\xi}_{A}+4 \mathscr{D}_{\rho} \bar{T}_{\mu \nu} \bar{\sigma}^{\mu \nu} \bar{\sigma}^{\rho} \xi_{A} & =M \bar{\xi}_{A},
\end{aligned}
$$

where the covariant derivatives are defined as follows:

$$
\begin{aligned}
& \mathscr{D}_{\mu} \xi_{A}=\left(\partial_{\mu}+\frac{1}{4} \Omega_{\mu}^{a b} \sigma_{a b}\right) \xi_{A}+i \xi_{B}\left(V_{\mu}\right)_{A}^{B}-\mathrm{i} \widetilde{V}_{\mu} \xi_{A} \\
& \mathscr{D}_{\mu} \bar{\xi}_{A}=\left(\partial_{\mu}+\frac{1}{4} \Omega_{\mu}^{a b} \bar{\sigma}_{a b}\right) \bar{\xi}_{A}+\mathrm{i} \bar{\xi}_{B}\left(V_{\mu}\right)_{A}^{B}+\mathrm{i} \widetilde{V}_{\mu} \bar{\xi}_{A}
\end{aligned}
$$

For the background in consideration, we require the $U(1)_{r}$-symmetry to be preserved, and thus, $T_{\mu \nu}$ and $\bar{T}_{\mu \nu}$ necessarily have to vanish, for they have nontrivial $U(1)_{r}$-charges given by \pm 2 . Thus, we have to set

$$
T_{\mu v}=\bar{T}_{\mu v}=0
$$

Then, in this case, using (22) and (23), Eqs. (24) and (25) can equivalently be written as ${ }^{69}$

$$
\begin{aligned}
(3 M+R) \xi_{A} & =2 \mathrm{i} \sigma^{\mu v}\left(V_{\mu v}\right)_{A}^{B} \xi_{B}+4 \mathrm{i} \sigma^{\mu \nu} \widetilde{V}_{\mu \nu} \xi_{A}, \\
(3 M+R) \bar{\xi}_{A} & =2 \mathrm{i} \bar{\sigma}^{\mu v}\left(V_{\mu \nu}\right)_{A}^{B} \bar{\xi}_{B}-4 \mathrm{i} \bar{\sigma}^{\mu v} \widetilde{V}_{\mu \nu} \bar{\xi}_{A} .
\end{aligned}
$$

Here, $\left(V_{\mu \nu}\right)_{A}^{B}$ and $\widetilde{V}_{\mu \nu}$ denote the field strengths of the respective R-symmetry gauge fields, and $R=\frac{6}{\ell^{2}}$ is the scalar curvature of $S^{3} \times S^{1}$. We pick a solution for which both the left- and right-hand sides are zero. Specifically, we have

$$
M=-\frac{R}{3},
$$

while the R-symmetry gauge fields have vanishing field strengths,

$$
\left(V_{\mu \nu}\right)_{A}^{B}=\widetilde{V}_{\mu \nu}=0 .
$$


However, we may, of course, turn on holonomies for $\left(V_{\mu}\right)_{A}$ and $\widetilde{V}_{\mu}$. For reasons that will become clear soon, we only consider a holonomy for $R+r$, in particular, we set

$$
\begin{aligned}
\widetilde{V}_{\mu} \mathrm{d} x^{\mu} & =-\frac{\alpha}{2 \ell} \mathrm{d} y, \\
\left(V_{\mu}\right)_{A}^{B} \mathrm{~d} x^{\mu} & =\frac{\alpha}{2 \ell} \mathrm{d} y\left(\tau_{3}\right)_{A}^{B},
\end{aligned}
$$

where $\tau_{3}$ is the third Pauli matrix. Note that by performing non-single-valued $(R+r)$ gauge transformations with parameter $e^{\mathrm{i} c y}$, for some $c \in \mathbb{R}$, we can arbitrarily shift the holonomy $\alpha$ and, in particular, make it zero. The latter is possible at the cost of introducing an extra $(R+r)$ monodromy ("twisting" in the sense of twisted sectors) around $S_{y}^{1}$. Recall that $\gamma$ in (17) parameterizes such a monodromy, i.e., the gluing cocycle for the $(R+r)$-symmetry flat bundle: as we go once around $S_{y}^{1}$, we identify fibers by an $e^{\mathrm{i} \gamma(R+r)}$ rotation. We can reverse the logic, and completely gauge away $\gamma$, and instead keep the holonomy $\alpha$ generic. We find the latter more convenient and adhere to such a convention below. We could try to similarly gauge away the $e^{\beta R}$ twisted sector in (17); however, this would introduce an imaginary background holonomy (manifesting that our background is non-unitary), which we find slightly inconvenient. Therefore, we work in a twisted sector with respect to the $R$ symmetry only. For any variable $X$ in our theory-be it a field or a Killing spinor-the periodicity is determined by its $\mathrm{R}$ charge, i.e.,

$$
X(y+\beta \ell)=e^{-\beta R} X(y) .
$$

Now, solving the remaining equations (22) and (23) and throwing away half of the solutions that cannot satisfy the twisted sector periodicity (due to having the wrong sign in front of $\frac{y}{2 \ell}$ in the exponent), we find the following set of Killing spinors:

$$
\begin{aligned}
& \xi_{1}=e^{-\frac{y}{2 \ell}-\mathrm{i} \alpha \frac{y}{\ell}} e^{\frac{\mathrm{i}}{2} \tau_{1} \theta} e^{\frac{\mathrm{i}}{2} \tau_{3}\left(\varphi+\tau+2 \zeta \frac{y}{\ell}\right)} \varepsilon, \\
& \bar{\xi}_{1}=e^{-\frac{y}{2 \ell}} e^{-\frac{\mathrm{i}}{2} \tau_{1} \theta} e^{\frac{\mathrm{i}}{2} \tau_{3}(\varphi-\tau)} \bar{\varepsilon}, \\
& \xi_{2}=e^{\frac{y}{2 \ell}} e^{-\frac{\mathrm{i}}{2} \tau_{1} \theta} e^{\frac{\mathrm{i}}{2} \tau_{3}(\varphi-\tau)} \eta, \\
& \bar{\xi}_{2}=e^{\frac{y}{2 \ell}+\mathrm{i} \alpha \frac{y}{\ell}} e^{\frac{i}{2} \tau_{1} \theta} e^{\frac{i}{2} \tau_{3}\left(\varphi+\tau+2 \zeta \frac{y}{\ell}\right)} \bar{\eta},
\end{aligned}
$$

where $\varepsilon, \bar{\varepsilon}, \eta, \bar{\eta}$ are constant two-component spinors. Part of these can still be broken due to the incorrect periodicity at generic $\alpha$ and $\zeta$, which will be discussed in more detail below.

Setting the deformations $\alpha=\zeta=0$, and if we replace $S^{1}$ by $\mathbb{R}$, we can think of these solutions as the flat space solutions conformally mapped to the cylinder $S^{3} \times \mathbb{R}$. This article allows us to easily identify the map between the conformal supercharges and the parameters in $\varepsilon, \bar{\varepsilon}, \eta, \bar{\eta}$ as follows:

$$
\begin{aligned}
\mathscr{Q}_{\alpha}^{1} & \longleftrightarrow \varepsilon_{\alpha}, \\
\widetilde{\mathscr{Q}}_{2 \dot{\alpha}} & \longleftrightarrow \bar{\varepsilon}^{\dot{\alpha}}, \\
\widetilde{\mathscr{S}}^{2 \dot{\alpha}} & \longleftrightarrow \eta^{\dot{\alpha}}, \\
\mathscr{S}_{1}^{\alpha} & \longleftrightarrow \bar{\eta}_{\alpha} .
\end{aligned}
$$

These are 8 out of 16 four-dimensional $\mathscr{N}=2$ conformal supercharges that commute with the combination $E-R$, and thus, their Killing spinors obey the twisted sector periodicity. The remaining supercharges, which are not commuting with $E-R$, acquire the wrong periodicity in the $y$ direction and are broken on $S^{3} \times S^{1}$.

One finds that the remaining eight preserved supercharges form an $\mathfrak{s u}(2 \mid 1)_{\ell} \oplus \mathfrak{s u}(2 \mid 1)_{r}$ superalgebra, which is centrally extended by the element $E-R$, with the nontrivial anti-commutation relations given by

$$
\begin{aligned}
\left\{\mathscr{Q}_{\alpha}^{1}, \mathscr{S}_{1}^{\beta}\right\} & =\frac{1}{2} \delta_{\alpha}^{\beta}(E-R)+\mathscr{M}_{\alpha}{ }^{\beta}-\delta_{\alpha}^{\beta} \frac{r+R}{2}, \\
\left\{\widetilde{\mathscr{S}}^{2 \dot{\alpha}}, \widetilde{\mathscr{Q}}_{2 \dot{\beta}}\right\} & =\frac{1}{2} \delta_{\dot{\beta}}^{\dot{\alpha}}(E-R)+\mathscr{M}^{\dot{\alpha}}{ }_{\dot{\beta}}+\delta_{\dot{\beta}}^{\dot{\alpha}} \frac{r-R}{2} .
\end{aligned}
$$

Here, $\mathscr{M}_{\alpha}{ }^{\beta}$ and $\mathscr{M}_{\dot{\beta}}^{\dot{\alpha}}$ are the generators of left- and right-rotations, respectively. In our conventions, $\mathscr{Q}_{\alpha}^{1}$ and $\mathscr{S}_{1}^{\alpha}$ generate the left factor, $\mathfrak{s u}(2 \mid 1)_{\ell}$, and $\widetilde{\mathscr{S}}^{2 \dot{\alpha}}$ together with $\widetilde{\mathscr{Q}}_{2 \dot{\beta}}$ generates the right factor, $\mathfrak{s u}(2 \mid 1)_{r}$. Note that if we turn on non-vanishing deformations $\alpha \neq 0$ or $\zeta \neq 0$, we further break part of the $\mathfrak{s u}(2 \mid 1)_{\ell} \oplus \mathfrak{s u}(2 \mid 1)_{r}$ supersymmetry. We will investigate this below.

Now, we proceed to present the four-dimensional $\mathscr{N}=2$ Yang-Mills and matter actions in our background. ${ }^{70}$ The four-dimensional $\mathscr{N}=2$ vector multiplet consists of a gauge field $A_{\mu}$ with the field strength $F_{\mu \nu}$, a complex scalar $\phi$, (anti)chiral gaugini $\left(\bar{\lambda}_{A}^{\dot{\alpha}}\right) \lambda_{A \alpha}$, and the 
auxiliary $S U(2)_{R}$-triplet $D_{A B}$. The $\mathscr{N}=2$ Yang-Mills action coupled to the rigid standard Weyl multiplet is then given by ${ }^{63-65}$

$$
\begin{gathered}
\mathscr{L}_{\mathrm{YM}}=\frac{1}{g_{\mathrm{YM}}^{2}} \operatorname{Tr}\left(\frac{1}{2} F_{\mu \nu} F^{\mu \nu}+4 \mathscr{D}_{\mu} \bar{\phi} \mathscr{D}^{\mu} \phi+\frac{2 R}{3} \bar{\phi} \phi-2 \mathrm{i} \lambda^{A} \sigma^{\mu} \mathscr{D}_{\mu} \bar{\lambda}_{A}+2 \lambda^{A}\left[\bar{\phi}, \lambda_{A}\right]+2 \bar{\lambda}^{A}\left[\phi, \bar{\lambda}_{A}\right]\right. \\
\left.+4[\phi, \bar{\phi}]^{2}-\frac{1}{2} D^{A B} D_{A B}\right)+\frac{\mathrm{i} \theta}{32 \pi^{2}} \operatorname{Tr}\left(\varepsilon^{\mu \nu \lambda \rho} F_{\mu \nu} F_{\lambda \rho}\right) .
\end{gathered}
$$

Here, $g_{\mathrm{YM}}$ is the four-dimensional Yang-Mills coupling, $R$ is the curvature of the background, $\theta$ is the $\theta$-angle, and the covariant derivatives are understood [see, e.g., (26) and (27)].

The four-dimensional $\mathscr{N}=2$ hypermultiplet $\left(q_{A I}, \psi_{I \alpha}, \bar{\psi}_{I}{ }_{I}^{\dot{\alpha}}, F_{\check{A} I}\right)$ contains the scalars $q_{A I}$, the fermions $\psi_{I \alpha}, \bar{\psi}_{I \alpha}$, and auxiliary fields $F_{\check{A} I}$. Here, $I, J, \ldots$ are flavor indices of the hypermultiplets, and $\varepsilon^{I J}$ is their pseudoreal structure. For instance, for a single free hypermultiplet, $I, J, \ldots=1,2$, and the flavor symmetry is $S U(2)_{F}$. More generally, $2 N_{f}$ half-hypermultiplets transform in the fundamental representation of the flavor symmetry group $U S p\left(2 N_{f}\right)_{F}$, part of which can be gauged by the dynamical vector multiplets. After gauging $G \subset U S p\left(2 N_{f}\right)_{F}$, the fundamental representation of $U S p\left(2 N_{f}\right)_{F}$ becomes some representation $\mathbf{R}$ of $G$, which is pseudoreal, in general, and for full hypermultiplets is of the form $\mathbf{R} \cong \mathscr{R} \oplus \overline{\mathscr{R}}$ in which case we say that hypermultiplets are valued in the (complex) representation $\mathscr{R}$. The action of (half)hypermultiplets (coupled to vector multiplets) including auxiliary fields is then given by ${ }^{71}$

$$
\begin{aligned}
\mathscr{L}_{\mathrm{mat}}= & \frac{1}{2} \mathscr{D}_{\mu} q^{A} \mathscr{D}^{\mu} q_{A}+q^{A}\{\phi, \bar{\phi}\} q_{A}+\frac{\mathrm{i}}{2} q^{A} D_{A B} q^{B}+\frac{2}{3} R q^{A} q_{A}-\frac{\mathrm{i}}{2} \overline{\psi \sigma}^{\mu} \mathscr{D}_{\mu} \psi \\
& -\frac{1}{2} \psi \phi \psi+\frac{1}{2} \bar{\psi} \bar{\psi} \bar{\psi}-q^{A} \lambda_{A} \psi+\bar{\psi} \bar{\lambda}_{A} q^{A}-\frac{1}{2} F^{\check{A}} F_{\check{A}},
\end{aligned}
$$

where we suppress $I, J, \ldots$ indices, and the covariant derivative for the scalars is given by

$$
\mathscr{D}_{\mu} q_{A I}=\partial_{\mu} q_{A I}-\mathrm{i}\left(A_{\mu}\right)_{I}^{J} q_{A J}+\mathrm{i} V^{B}{ }_{A} q_{B I} .
$$

The vector multiplet and hypermultiplet actions in (39) and (40) are invariant under $\mathscr{N}=2$ supersymmetry. The vector multiplet components transform as follows:

$$
\begin{aligned}
Q A_{\mu}= & \mathrm{i} \xi^{A} \sigma_{\mu} \bar{\lambda}_{A}-\mathrm{i} \bar{\xi}^{A} \bar{\sigma}_{\mu} \lambda_{A}, \\
Q \phi= & -\mathrm{i} \xi^{A} \lambda_{A}, \\
Q \bar{\phi}= & -\mathrm{i} \bar{\xi}^{A} \bar{\lambda}_{A}, \\
Q \lambda_{A}= & \frac{1}{2} \sigma^{\mu v} \xi_{A} F_{\mu \nu}+2 \sigma^{\mu} \bar{\xi}_{A} \mathscr{D}_{\mu} \phi+\sigma^{\mu} \mathscr{D}_{\mu} \bar{\xi}_{A} \phi-2 \mathrm{i} \xi_{A}[\phi, \bar{\phi}]+D_{A B} \xi^{B}, \\
Q \bar{\lambda}_{A}= & \frac{1}{2} \bar{\sigma}^{\mu v} \bar{\xi}_{A} F_{\mu \nu}-2 \bar{\sigma}^{\mu} \xi_{A} \mathscr{D}_{\mu} \bar{\phi}-\bar{\sigma}^{\mu} \mathscr{D}_{\mu} \xi_{A} \bar{\phi}+2 \mathrm{i} \bar{\xi}_{A}[\phi, \bar{\phi}]+D_{A B} \bar{\xi}^{B}, \\
Q D_{A B}= & -\mathrm{i} \bar{\xi}_{A} \bar{\sigma}^{\mu} \mathscr{D}_{\mu} \lambda_{B}-\mathrm{i} \bar{\xi}_{B} \bar{\sigma}^{\mu} \mathscr{D}_{\mu} \lambda_{A}+\mathrm{i} \xi_{A} \sigma^{\mu} \mathscr{D}_{\mu} \bar{\lambda}_{B}+\mathrm{i} \xi_{B} \sigma^{\mu} \mathscr{D}_{\mu} \bar{\lambda}_{A} \\
& -2\left[\phi, \bar{\xi}_{A} \bar{\lambda}_{B}+\bar{\xi}_{B} \bar{\lambda}_{A}\right]-2\left[\bar{\phi}, \xi_{A} \lambda_{B}+\xi_{B} \lambda_{A}\right] .
\end{aligned}
$$

The gauge field $A_{\mu}$ is real, the reality condition of the scalar reads $\phi^{*}=\bar{\phi}$, and the auxiliary fields satisfy

$$
\left(D_{A B}\right)^{*}=-e^{-\frac{y}{\ell}\left(s_{A}+s_{B}\right)} D^{A B}=-e^{-\frac{y}{\ell}\left(s_{A}+s_{B}\right)} \varepsilon^{A \widetilde{A}} \varepsilon^{B \widetilde{B}} D_{\widetilde{A B}},
$$

where $s_{1}=1$ and $s_{2}=-1$. Here, the factor $e^{-\frac{y}{\ell}\left(s_{A}+s_{B}\right)}$ has to be added because we work in a twisted sector (35), where the $y$-periodicity of fields is dependent on their R-charge.

Similarly, the hypermultiplet supersymmetry transformations are given by

$$
\begin{aligned}
& Q q_{A}=-\mathrm{i} \xi_{A} \psi+\mathrm{i} \bar{\xi}_{A} \bar{\psi} \\
& Q \psi=2 \sigma^{\mu} \bar{\xi}_{A} \mathscr{D}_{\mu} q^{A}+\sigma^{\mu} \mathscr{D}_{\mu} \bar{\xi}_{A} q^{A}+4 \mathrm{i} \xi_{A} \bar{\phi} q^{A}+2 \check{\xi}_{\check{\check{A}}} F^{\check{A}}, \\
& Q \bar{\psi}=2 \bar{\sigma}^{\mu} \xi_{A} \mathscr{D}_{\mu} q^{A}+\bar{\sigma}^{\mu} \mathscr{D}_{\mu} \xi_{A} q^{A}-4 \mathrm{i} \bar{\xi}_{A} \phi q^{A}+2 \bar{\xi}_{\check{A}} F^{\check{A}}, \\
& Q F_{\check{A}}=\mathrm{i} \check{\xi}_{\check{A}} \sigma^{\mu} \mathscr{D} \mu \bar{\psi}-2 \check{\xi}_{\check{A}} \phi \psi-2 \check{\xi}_{\check{A}} \lambda_{B} q^{B}-\mathrm{i} \bar{\zeta}_{\check{A}} \bar{\sigma}^{\mu} \mathscr{D} \mu \psi \\
& -2 \overline{\check{\xi}}_{\bar{A}} \bar{\phi} \bar{\psi}+2 \overline{\check{\xi}}_{\bar{A}} \bar{\lambda}_{B} q^{B} \text {. }
\end{aligned}
$$

We impose the following reality conditions for the bosonic fields of the hypermultiplets: 


$$
\begin{aligned}
& \left(q_{A I}\right)^{*}=e^{-\frac{y}{\ell} s_{A}} q^{A I} \equiv e^{-\frac{y}{\ell} s_{A}} \varepsilon^{A B} \varepsilon^{I J} q_{B J}, \\
& \left(F_{\check{A} I}\right)^{*}=-e^{-\frac{y}{\ell} s_{\breve{A}}} F^{\check{A} I} \equiv-e^{-\frac{y}{\ell} s_{\breve{A}} \check{E} \varepsilon^{\check{A} \check{B}} \varepsilon^{I J} F_{\breve{B} J},}
\end{aligned}
$$

where again $s_{1,2}= \pm 1$ accounts for the twisted sector. Note that we have introduced the same twisted sector for the auxiliary $S U(2)$ index $\check{A}$ as for the $S U(2)_{R}$ index $A$-this is because in the rest of this paper, it will be convenient to identify $A$ with $A$.

Finally, we stress that we assume our matter content to always satisfies the $U(1)_{r}$-anomaly cancellation condition, which, incidentally, also ensures that the theory is conformal and free of global anomalies. For each simple factor $G_{i}$ of the gauge group, this condition reads ${ }^{72}$

$$
2 h^{\vee}\left(G_{i}\right)=\sum_{x} C\left(\mathbf{R}_{i, x}\right) \prod_{j \neq i} \operatorname{dim} \mathbf{R}_{j, x}+\sum_{y} 2 C\left(\mathscr{R}_{i, y}\right) \prod_{j \neq i} \operatorname{dim} \mathscr{R}_{j, y},
$$

where $h^{\vee}$ is the dual Coxeter number, $C(\mathscr{R})$ is an index of the representation $\mathscr{R}$, and the sum over $y$ goes over all full hypermultiplets $\mathbf{R}_{y}=\mathscr{R}_{y} \oplus \overline{\mathscr{R}}_{y}$, whereas the sum over $x$ goes over the remaining half-hypermultiplets $\mathbf{R}_{x}$. Furthermore, by $\mathbf{R}_{i, x}$, we denote the representation of the $x$ th half-hypermultiplet under the simple gauge group factor $G_{i}$, and similarly, by $\mathbf{R}_{i, y}=\mathscr{R}_{i, y} \oplus \overline{\mathscr{R}}_{i, y}$, we denote the representation of the $y$ th full hypermultiplet under $G_{i}$. For Abelian groups, $h^{\vee}=0$, and thus, (46) implies that Abelian factors are decoupled and free. Hence, they are not interesting and excluded from our discussion.

\section{Fugacities and the supersymmetry they preserve}

Let us now establish the correspondence between fugacities of the superconformal index and the supersymmetry algebra preserved by our background in their presence. To make statements less cumbersome, we drop the characterization "centrally extended," as all supersymmetry algebras we deal with are centrally extended by $E-R$.

In the presence of non-vanishing $\alpha$ and $\zeta$ in (36), part of the solutions in (36) are broken on $S^{3} \times S_{y}^{1}$ due to the spinors having incorrect periodicity upon going around $S_{y}^{1}$. Indeed, in the twisted sector (35), our spinors ought to satisfy the following conditions:

$$
\begin{array}{ll}
\xi_{1}(y+\beta \ell)=e^{-\frac{\beta}{2}} \xi_{1}(y), & \bar{\xi}_{1}(y+\beta \ell)=e^{-\frac{\beta}{2}} \bar{\xi}_{1}(y), \\
\xi_{2}(y+\beta \ell)=e^{\frac{\beta}{2}} \xi_{2}(y), & \bar{\xi}_{2}(y+\beta \ell)=e^{\frac{\beta}{2}} \bar{\xi}_{2}(y) .
\end{array}
$$

Thus, $\xi_{1}$ and $\bar{\xi}_{2}$ are generically broken in (36), as they do not obey this periodicity, whereas $\bar{\xi}_{1}$ and $\xi_{2}$ are preserved. Therefore, only the $\mathfrak{s u}(2 \mid 1)_{r}$ inside $\mathfrak{s u}(2 \mid 1)_{\ell} \oplus \mathfrak{s u}(2 \mid 1)_{r}$ is unbroken, and we conclude as follows:

- Keeping all three fugacities $(\beta, \zeta, \alpha)$-or, equivalently, $(p, q, t)$ of $(12)$-in the index (17) only preserves $\mathfrak{s u}(2 \mid 1)_{r}$.

If we additionally want to preserve $\mathfrak{s u}(2 \mid 1)_{\ell}$, we have to ensure that all conformal Killing spinors in (36) satisfy the periodicity conditions (47). This is attained if and only if

$$
\alpha=\frac{2 \pi N}{\beta} \text { and } \zeta=\frac{2 \pi M}{\beta}, \quad M, N \in \mathbb{Z}
$$

Recall that, by an $(R+r)$-symmetry gauge transformation, we could equivalently consider $\gamma=2 \pi N / \beta$ instead. The value $M=N=0$ corresponds to specializing (17) to

$$
\mathscr{I}(\beta)=\operatorname{Tr}_{\mathscr{H}_{S^{3}}}(-1)^{F} e^{-\beta(E-R)},
$$

which is the standard "Schur" specialization of the superconformal index. ${ }^{47,48}$ Thus, we draw yet another general conclusion as follows:

- The Schur index specialization preserves $\mathfrak{s u}(2 \mid 1)_{\ell} \oplus \mathfrak{s u}(2 \mid 1)_{r}$.

Allowing in (48) for non-zero integer $M \neq 0$ corresponds to an insertion of $e^{4 \pi \mathrm{i} M j_{1}}$ into the Schur index (49). Since $j_{1}$ is half-integral, such an insertion is immaterial to the index. However, a non-zero integer $N \neq 0$ in (48) corresponds to an insertion of $e^{2 \pi \mathrm{i} N(R+r)}$ into the Schur index, which can be non-trivial: because in a Lagrangian theory $R+r$ is half-integral, the insertion of $e^{2 \pi \mathrm{i} N(R+r)} \operatorname{depends}$ on $N$ mod 2 and thus becomes nontrivial for $N \equiv 1 \bmod 2$. (As explained in Ref. 78, this is also true in general superconformal field theory.) This is a tiny quirk of the index to which we will return in Sec. II E. In fact, in our localization computations, we will keep $M$ and $N$ generic.

Interestingly, there is an additional, intermediate case, where we tune $\zeta=\alpha .{ }^{73}$ In this case, the two competing phases in (36) cancel each other for the solutions corresponding to $\mathscr{Q}_{-}^{1}$ and $\mathscr{S}_{1}^{-}$. These two generate the $\mathfrak{s u}(1 \mid 1)_{\ell}$ subalgebra of $\mathfrak{s u}(2 \mid 1)_{\ell}$. It is easy to check that $\alpha=\zeta$ corresponds to turning on the fugacity for $2 j_{1}+R+r$, which-upon shifting by $\widetilde{\delta}_{1-}=E-2 j_{2}-2 R+r$ and redefinition of the fugacity for $E-R$-is equivalent to introducing an additional fugacity " $s$ " for the symmetry

$$
\mathscr{Z}=j_{1}-j_{2}+r .
$$

That is, such a specialization of the background computes 


$$
\operatorname{Tr}(-1)^{F} q^{E-R} s^{\mathscr{Z}}
$$

We will argue soon that $s$ actually cancels, and this again reduces to the Schur index. Thus, we conclude as follows:

- The $\zeta=\alpha$ specialization preserves $\mathfrak{s u}(1 \mid 1)_{\ell} \oplus \mathfrak{s u}(2 \mid 1)_{r}$ but still computes the Schur index.

We can also contemplate the possibility of setting $\zeta=-\alpha$ in which case the surviving left-handed supercharges are $\mathscr{Q}_{+}^{1}$ and $\mathscr{S}_{1}^{+}$. However, they are not of interest for the purpose of this paper.

Finally, as we briefly touch upon in Sec. II F, one can further refine the Schur index by the insertion of an $\mathscr{N}=(2,2)$-supersymmetric surface defect at $\theta=0$, extended along $\tau$ and $y$. Such a defect must preserve $\mathfrak{s u}(1 \mid 1)_{\ell} \oplus \mathfrak{s u}(1 \mid 1)_{r}$, which is still enough for all the constructions discussed in the current paper to work.

\section{The chiral algebra}

In this section, let us assume for simplicity that $M=N=0$, that is, $\zeta=\alpha=0$, and so we deal with the pure Schur index background. We will comment on non-zero $M$ and $N$ toward the end of this section and will work in such a more general setting later on.

We note that the two supercharges relevant for the chiral algebra construction (Ref. 9),

$$
\begin{aligned}
& \mathbb{Q}_{1}=\mathscr{Q}_{-}^{1}+\frac{1}{\ell} \widetilde{\mathscr{S}}^{2-}, \\
& \mathbb{Q}_{2}=\frac{1}{\ell} \mathscr{S}_{1}^{-}-\widetilde{\mathscr{Q}}_{2-},
\end{aligned}
$$

belong to the algebra $\mathfrak{s u}(2 \mid 1)_{\ell} \oplus \mathfrak{s u}(2 \mid 1)_{r}$ described above. Furthermore, they also belong to the smaller subalgebra $\mathfrak{s u}(1 \mid 1)_{\ell} \oplus \mathfrak{s u}(2 \mid 1)_{r}($ or even $\left.\mathfrak{s u}(1 \mid 1)_{\ell} \oplus \mathfrak{s u}(1 \mid 1)_{r}\right)$ mentioned at the end of Subsection II C. Because of relation

$$
\left\{\mathbb{Q}_{1}, \mathbb{Q}_{2}\right\}=-\frac{1}{\ell} \mathscr{Z}=\frac{1}{\ell}\left(j_{2}-j_{1}-r\right),
$$

any operator in the cohomology of $\mathbb{Q}_{i}$-or in the equivariant cohomology of $\mathscr{Q}^{H}=\mathbb{Q}_{1}+\mathbb{Q}_{2}$-necessarily satisfies $\mathscr{Z}=0$. This immediately shows that the additional fugacity $s$ for the $\mathscr{Z}$ symmetry in (51) indeed cancels: only observables in the cohomology of $\mathscr{Q}^{H}$ contribute to the index, ${ }^{74}$ and they have $\mathscr{Z}=0$.

Let us proceed to explore the structure of $\mathbb{Q}_{i}$ - or $\mathscr{Q}^{H}$-cohomology. We note that the supercharge $\mathscr{Q}^{H}$ is, of course, the dimensional uplift of an analogous supercharge in three-dimensional $\mathscr{N}=4$ theories studied in Ref. 50. It is therefore suggestive to view the two-dimensional chiral algebra construction as the dimensional uplift of the one-dimensional topological quantum field theory construction studied in that reference.

We introduce the following rotation generators:

$$
\begin{aligned}
\mathscr{M}^{\perp} & =\mathscr{M}_{+}{ }^{+}-\mathscr{M}^{+}{ }_{+}=P_{\tau}, \\
\mathscr{M} & =\mathscr{M}_{+}{ }^{+}+\mathscr{M}^{+}{ }_{+}=P_{\varphi},
\end{aligned}
$$

where, akin to the notation in Refs. 50 and 75, by writing $P_{\tau}$ and $P_{\varphi}$, we emphasize that these generators simply act by rotating the $\tau$ - and $\varphi$-circles in our fibration coordinates on $S^{3}$, respectively. Next, we introduce the twisted $\varphi$-rotation,

$$
\widehat{P}_{\varphi}=P_{\varphi}+R,
$$

which, again in accordance with the three-dimensional case, generates a $\mathscr{Q}^{H}$-closed operation. However, unlike in three dimensions, this operation is not $\mathscr{Q}^{H}$-exact. Additionally, in our four-dimensional case, we also have a "twisted time translation," given by the central element of the algebra

$$
H=E-R,
$$

which generates twisted translations in the $S_{y}^{1}$ direction of the $S^{3} \times S_{y}^{1}$ geometry. Being a central charge, it is, of course, $\mathscr{Q}^{H}$-closed, but it is not $\mathscr{Q}^{H}$-exact. Following Ref. 9, we consider the holomorphic and anti-holomorphic linear combinations of these and find the latter to be $\mathbb{Q}_{i}$-and thus $\mathscr{Q}^{H}$-exact,

$$
\begin{aligned}
2 L_{-1} & =H+\widehat{P}_{\varphi}=E+P_{\varphi}, \\
2 \bar{L}_{-1} & =H-\widehat{P}_{\varphi}=E-P_{\varphi}-2 R \\
& =\left\{\mathbb{Q}_{1}, \mathscr{S}_{1}^{-}+\widetilde{\mathscr{Q}}_{2-}\right\}=\left\{\mathbb{Q}_{2}, \ell \mathscr{Q}_{-}^{1}-\widetilde{\mathscr{S}}^{2-}\right\}=\left\{\mathscr{Q}^{H}, \mathscr{S}_{1}^{-}+\widetilde{\mathscr{Q}}_{2}-\right\} .
\end{aligned}
$$


This readily implies, as in Ref. 9, that the $\mathscr{Q}^{H}$-cohomology has the structure of a chiral algebra or a vertex operator algebra (VOA), as we call them interchangeably in this paper.

More precisely, because $\left(\mathscr{Q}^{H}\right)^{2}=\left\{\mathbb{Q}_{1}, \mathbb{Q}_{2}\right\}=\frac{1}{\ell}\left(\mathscr{M}^{\perp}+r\right)$, the chiral algebra is going to live at the fixed point locus of $\mathscr{M}^{\perp}$. This is a torus located at $\theta=\pi / 2$ and parameterized by our coordinates $\varphi$ and $y$-we often refer to it as $S_{\varphi}^{1} \times S_{y}^{1}$. Recall that $E$ is a dilatation generator, which acts as $E=\Delta+r \partial_{r}$ in flat space on observables of conformal dimension $\Delta$. Then, upon moving to $S^{3} \times \mathbb{R}$ and $S^{3} \times S_{y}^{1}$, it becomes the operator $\ell \partial_{y}$ [the additional numeric term $\Delta$ disappears because $\mathscr{O}^{\text {cylinder }}=(r / \ell)^{\Delta} \mathscr{O}^{\text {flat }}$ ]. Similarly, on $S^{3} \times S_{y}^{1}$, we have that $P_{\varphi}=-\mathrm{i} \partial_{\varphi}$. Note that

$$
\begin{aligned}
& \ell \frac{\partial}{\partial y}-\mathrm{i} \frac{\partial}{\partial \varphi}=2 \partial_{w} \\
& \ell \frac{\partial}{\partial y}+\mathrm{i} \frac{\partial}{\partial \varphi}=2 \partial_{\bar{w}}
\end{aligned}
$$

where we have introduced the coordinates $w$ and $\bar{w}$ as

$$
w=\frac{y}{\ell}+\mathrm{i} \varphi, \quad \bar{w}=\frac{y}{\ell}-\mathrm{i} \varphi .
$$

In the case when $S_{y}^{1}$ is replaced by $\mathbb{R}$, these are precisely coordinates on the cylinder related to the flat space ones via the usual map

$$
z=e^{w}, \quad \bar{z}=e^{\bar{w}}
$$

Recall that the chiral algebra is formed by the twisted-translated Schur operators. ${ }^{9}$ These are defined as the ordinary Schur operators translated along the "chiral algebra surface" by employing the operators $L_{-1}$ and $\bar{L}_{-1}$ as translation generators. On $S_{\varphi}^{1} \times S_{y}^{1} \subset S^{3} \times S_{y}^{1}$, the twisted-translated Schur operators are constructed as follows:

$$
\mathscr{O}(w, \bar{w})=\mathscr{O}_{A_{1} \ldots A_{n}} u^{A_{1}} \ldots u^{A_{n}}, \quad \text { where } \quad u^{A}=\left(e^{-\bar{w} / 2}, e^{\bar{w} / 2}\right)
$$

Here, the $S U(2)_{R}$ highest weight component $\mathscr{O}_{+} \ldots+$ is the usual Schur operator, while $\mathscr{O}(w, \bar{w})$ is its twisted-translated cousin. With this definition, it is straightforward to check that

$$
\begin{array}{ll}
\partial_{w} \mathscr{O}(w, \bar{w})=\frac{1}{2}\left[E+P_{\varphi}, \mathscr{O}(w, \bar{w})\right] & =\left[L_{-1}, \mathscr{O}(w, \bar{w})\right], \\
\partial_{\bar{w}} \mathscr{O}(w, \bar{w})=\frac{1}{2}\left[E-P_{\varphi}-2 R, \mathscr{O}(w, \bar{w})\right] & =\left[\bar{L}_{-1}, \mathscr{O}(w, \bar{w})\right],
\end{array}
$$

where the latter is $\mathbb{Q}_{i}$-exact and thus $\mathscr{Q}^{H}$-exact because $\bar{L}_{-1}$ is exact and $\mathscr{O}(w, \bar{w})$ is closed. As usual, it implies that in the $\mathbb{Q}_{i}$ or $\mathscr{Q}^{H}$ cohomology, $\partial_{\bar{w}} \mathscr{O}(w, \bar{w})$ is trivial, and so the cohomology classes

$$
\mathscr{O}(w)=[\mathscr{O}(w, \bar{w})]
$$

are holomorphic in $w$ and, furthermore, have the VOA structure induced by the four-dimensional OPE.

In fact, by a Weyl transformation, one can straightforwardly connect our definitions to the flat space description of Ref. 9 and see that at the level of an abstract VOA, one has

$$
\mathscr{O}^{\mathrm{cyl}}(w)=z^{h} \mathscr{O}^{\mathrm{flat}}(z)
$$

where the superscripts "cyl" and "flat" are there to distinguish the construction on a cylinder $S^{3} \times \mathbb{R}$ from the flat space one of Ref. 9. Furthermore, $h$ is the (holomorphic) conformal dimension of a VOA operator $\mathscr{O}$, which is related to the quantum numbers of the Schur operator $\mathscr{O}_{+\cdots+}$ by $^{9}$

$$
h=R+j_{1}+j_{2} \text {. }
$$

Finally, note that the above construction admits a generalization to non-zero $\zeta=2 \pi M / \beta$ and $\alpha=2 \pi N / \beta$. The parameter $\zeta$, being a geometric twist of the background, modifies the "time translation" generator $E$ to $E-\mathrm{i} \zeta P_{\varphi}-\mathrm{i} \zeta P_{\tau}$. This amounts to shifting the complex

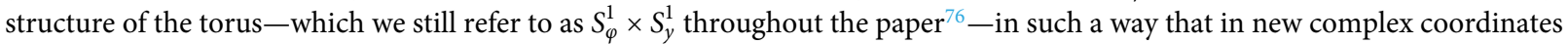

$$
w=\frac{y}{\ell}+\mathrm{i} \varphi+\mathrm{i} \zeta \frac{y}{\ell}, \quad \bar{w}=\frac{y}{\ell}-\mathrm{i} \varphi-\mathrm{i} \zeta \frac{y}{\ell},
$$

the two-dimensional theory looks the same. Specifically, the $\partial_{\bar{w}}$ translation is $\mathscr{Q}^{H}$-exact, and the cohomology has the emergent VOA structure. This shift, however, might alter the periodicity in the $y$ direction. The latter is also true for non-zero $\alpha=2 \pi N / \beta$, which, despite not being a 
geometric parameter of the four-dimensional background, acquires an emergent geometric interpretation in two dimensions. In the following, we will perform the localization with general non-zero $M$ and $N$, but before doing so, we first give a more unified view on the discrete refinements of the construction.

\section{E. Spin structures on $S_{\varphi}^{1} \times S_{y}^{1}$}

Since $R, j_{1}$, and $j_{2}$ are either integers or half-integers, relation (66) implies that all vertex operators in the chiral algebra have either integral or half-integral spins. Whenever half-integral spins are present, one has to choose a spin structure. In the case at hand, we ought to consider the spin structure on the torus $S_{\varphi}^{1} \times S_{y}^{1}$ on which our chiral algebra lives. We would like to stress that generally in the SCFT/VOA correspondence, we find a $\frac{1}{2} \mathbb{Z}$-graded super-VOA (SVOA), even though for simplicity of notations we call it VOA. The "super" refers to the $\mathbb{Z}_{2}$-grading-the fermion parity-inherited from the four-dimensional theory. The $\frac{1}{2} \mathbb{Z}$-grading is the two-dimensional conformal grading. Unlike in unitary VOAs appearing in the context of two-dimensional CFT, where the spin-statistics theorem relates the two gradings, in our setting, the $\frac{1}{2} \mathbb{Z}$ and $\mathbb{Z}_{2}$ gradings are not related. It is the $\frac{1}{2} \mathbb{Z}$ grading by the spin that plays the role in the choice of spin structure, and one can indeed pick a non-trivial spin structure whenever the VÖA contains operators of half-integral degree.

As a matter of fact, the construction from Subsection II D provides a natural choice of spin structure: On the one hand, the chiral algebra is periodic in the $y$ direction, that is, spinors have Ramond boundary conditions along $S_{y}^{1}$. This is simply the reflection of the fact that our theory is defined in a twisted sector, where the R-charge dependent non-periodicity of $\mathscr{O}_{A_{1} \ldots A_{n}}$ is canceled by explicit non-periodic factors $u^{A_{1}} \ldots u^{A_{n}}$. This can alternatively be understood as a consequence of the fact that the twisted $y$-translations are generated by $E-R$, which is a symmetry of our system. On the other hand, the $S_{\varphi}^{1}$ direction has NS sector boundary conditions. It is most straightforward to see this from Eq. (65): since vertex operators are single-valued in flat space, they are in the NS sector on the cylinder. Indeed, Eq. (65) implies that on the cylinder, the $\varphi$-periodicity is determined by

$$
z^{h}=e^{\frac{y}{\ell} h+\mathrm{i} \varphi h},
$$

and while operators with integral $h$ are periodic, those with half-integral $h$ are anti-periodic. One can alternatively check this using the explicit definition (62). Thus, in conclusion, the chiral algebra construction provides us with a natural choice of spin structure on $S_{\varphi}^{1} \times S_{y}^{1}$, which is in the NS sector along $S_{\varphi}^{1}$ and in the R sector along $S_{y}^{1}$; we will refer to this choice as $(1,0)$.

However, we realize that there exists a simple modification of the original construction, which flips the spin structure to $(1,1)$. Recall that in Subsection II C, we noted that the Schur index could be modified by an insertion of $e^{2 \pi i N(R+r)}$, which did not break any supersymmetry and only depended on $N \bmod 2$. In particular, it is nontrivial for $N=1$, corresponding to an insertion of

$$
(-1)^{2(R+r)} \text {. }
$$

Such an insertion can be realized by turning on an $(R+r)$-holonomy in our background, and it flips the $y$-periodicity for those operators that have half-integral $R+r$. The operators that contribute to this slightly modified index are still the Schur operators because such a refinement preserves $\mathscr{Q}^{H}$. In particular, they still obey $Z=j_{1}-j_{2}+r=0$, and using (66), we can find another expression for the chiral algebra conformal dimension,

$$
h=R+r+2 j_{1} \text {. }
$$

This equation clearly shows that $R+r$ is (half-)integral if and only if $h$ is (half-)integral. ${ }^{78}$ Hence, the insertion of (69) changes the periodicity along $y$ for all operators that have half-integral $h$. In other words, the modification (69) flips the spin structure along $S_{y}^{1}$.

Finally, there also exists a way to flip the $S_{\varphi}^{1}$ spin structure, though it does not follow from any of our earlier discussions yet. Similar to how we were able to flip the $S_{y}^{1}$ spin structure, it must be possible to achieve this by turning on the monodromy $(-1)^{2(R+r)}$ for the $S_{\varphi}^{1}$ circle. Since this circle is contractible, this ought to be done by an insertion of a codimension-two defect with non-zero vorticity for the $R+r$ symmetry. [Codimension-two defects characterized by a vortex-type singularity have recently been a subject of increasing attention (see, e.g., Refs. 144, 146, 147, 149, and 156-158).] Since this surface defect is expected to change the NS boundary condition along $S_{\varphi}^{1}$ into the R one, at the level of $\mathscr{Q}^{H}$ cohomology, it is expected to give the Ramond sector module(s) of the VOA (that is, twisted modules). We will discuss it in Subsection II F.

In this subsection, we summarize the following:

1. The chiral algebra on $S_{\varphi}^{1} \times S_{y}^{1}$ comes with the natural choice of the $(1,0)$ spin structure, where spinorial vertex operators are anti-periodic along $S_{\varphi}^{1}$ and periodic along $S_{y}^{1}$.

2. We can flip the $S_{y}^{1}$ spin structure by turning on the holonomy $(-1)^{2(R+r)}$ along $S_{y}^{1}$.

3. We can flip the $S_{\varphi}^{1}$ spin structure by the insertion of an $(R+r)$ monodromy-creating surface defect at $\theta=0$.

In fact, the choice of spin structure is intimately related to the modular properties of the chiral algebra characters. We will discuss some aspects of it later in this work while leaving a detailed investigation of this connection for the future. Now, let us look closer at the defect mentioned in the third item above. 


\section{F. The canonical R-symmetry interface and surface defects}

The possibility to refine the Schur index by an extra factor of $e^{2 \pi \mathrm{i}(R+r)}=(-1)^{2(R+r)}$, as discussed in Subsection II E and in Sec. II C, was attributed to the possibility of turning on the background holonomy $\alpha=2 \pi / \beta$ for $R+r$, without breaking any supersymmetry. In Sec. II C, it was equivalently interpreted as a twisted sector for the $U(1)_{R+r}$ symmetry, with the twisting parameter $\gamma=2 \pi / \beta$. In the latter case, we can think of it as placing a codimension-one symmetry defect at any fixed point $\mathrm{pt} \in S_{y}^{1}$ (and extending it in the $S^{3}$ directions) that implements the $(-1)^{2(R+r)}$ transformation. We are going to call this symmetry defect a canonical R-symmetry codimension-one defect (or interface) because it plays an important role in various constructions.

For arbitrary operators in a general superconformal field theory, the value of $R+r$ does not necessarily possess any nice properties. However, we have seen in (70) that for Schur operators, $R+r \in \frac{1}{2} \mathbb{Z}$. Hence, $(-1)^{2(R+r)}$ becomes a $\mathbb{Z}_{2}$ defect in the Schur sector. In Lagrangian theories (which are the cases of primary interest in this paper), $R+r \in \frac{1}{2} \mathbb{Z}$ for all operators, and $(-1)^{2(R+r)}$ is a $\mathbb{Z}_{2}$ symmetry defect in the full theory.

As is well-known, the Schur sector, in particular, the chiral algebra construction, admits a refinement by $\mathscr{N}=(2,2)$ surface defects orthogonal to the chiral algebra plane and intersecting it at the origin ${ }^{9}$ (see also Refs. 18, 23, 37, and 54). In our $S^{3} \times S_{y}^{1}$ geometry, such defects no longer intersect the chiral algebra torus: they are extended along the torus located at

$$
\theta=0: \quad \text { surface defect extended along } S_{\tau}^{1} \times S_{y}^{1} .
$$

With the canonical R-symmetry interface at hand, we can consider more general configurations with the R-symmetry interface ending at the surface defect. Geometrically, we place the R-symmetry interface at any fixed value of $\varphi$ and extend it in the $\theta, \tau$, and $y$ directions, ending it at the surface defect sitting at $\theta=0$. Following the nomenclature of Ref. 23, the latter type of defects living at the boundary of the R-symmetry interface will be referred to as the "twisted" or "monodromy" surface defects. They have the distinguishing property that operators with half-integral $R+r$ have monodromy $(-1)$ around such defects.

Recall from (70) that $R+r$ is (half-)integral whenever $h$ is (half-)integral. Therefore, only operators that have half-integral $h$ receive the monodromy $(-1)$ around the twisted defect. Thus, at the level of VOA, such defects will change the spin structure of $S_{\varphi}^{1}$ from NS to R, and they correspond to the twisted, or "Ramond sector," modules.

The above discussion is completely general and holds for arbitrary four-dimensional $\mathscr{N}=2$ superconformal field theories: in such theories, all our twisted surface defects of interest would sit at the end of the topological R-symmetry interface defined above. To further specify the surface defect, one ought to provide more details on what exactly happens at the boundary of the R-symmetry interface. Since our focus here is on Lagrangian theories, we are going to provide more details for this case.

First, we note that for all fields in the vector multiplet, $(-1)^{2(R+r)} \equiv 1$. Hence, the R-symmetry interface is completely transparent and can be ignored for the vector multiplets.

However, for all fields in the hypermultiplet, the opposite is true, i.e., $(-1)^{2(R+r)} \equiv-1$. Note that this is the same as the flavor symmetry interface for the hypermultiplets, which performs the $(-1) \in U(1)_{F} \subset S U(2)_{F}$ flavor symmetry transformation across the defect. The "flavor monodromy" defects for free hypermultiplets were considered in Ref. 23 and, indeed, were used there to define the spectral flow connecting the NS and R sectors in the $\beta-\gamma$ system. We thus see that for Lagrangian theories, our R-symmetry interface coincides with the flavor interface of Ref. 23 for the transformation $(-1) \in U(1)_{F}$. However, the two types of defects are members of different families: while the defect of Ref. 23 admits a continuous generalization to $e^{\mathrm{i} \alpha} \in U(1)_{F}$ in the hypermultiplet theory, our R-symmetry defect is always discrete but can be defined in an arbitrary $\mathscr{N}=2$ superconformal field theory.

We proceed to define the simplest monodromy surface defects in a Lagrangian theory. Because the R-symmetry interface ending on them is not visible to vector multiplets, we define the simplest surface defects to be invisible for vector multiplets as well. As for the hypermultiplets, the detailed definition involving specifying the asymptotic behavior of all fields at the defect location is given in Appendix D. Such a choice of asymptotic behavior breaks half of the supersymmetry, and in Appendix D, we define the defects in a way that preserves the following four supercharges:

$$
\text { preserved supercharges: } \quad \mathscr{Q}_{-}^{1}, \mathscr{S}_{1}^{-}, \widetilde{\mathscr{Q}}_{2-}, \widetilde{\mathscr{S}}^{2-} \text {, }
$$

forming the $\mathfrak{s u}(1 \mid 1)_{\ell} \oplus \mathfrak{s u}(1 \mid 1)_{r}$ subalgebra of $\mathfrak{s u}(2 \mid 1)_{\ell} \oplus \mathfrak{s u}(2 \mid 1)_{r}$. It can be interpreted as the $\mathscr{N}=(2,2)$ supersymmetry on $S_{\varphi}^{1} \times S_{y}^{1}$.

Note that the supercharges (72) are exactly the ones required for the chiral algebra construction. In particular, this means that the $\mathscr{Q}^{H}$ is still preserved, and its cohomology classes are still represented by the twisted-translated Schur operators. The index in the presence of a defect still counts Schur operators, albeit again in a slightly modified way. In Appendix D, we actually define two such simplest defects that correspond to the two Ramond modules of the symplectic boson. ${ }^{79}$

\section{LOCALIZATION}

In this section, we move toward applying supersymmetric localization to (Lagrangian) four-dimensional $\mathscr{N}=2$ superconformal field theories in order to derive the two-dimensional chiral algebra action. We will explain certain crucial points along the way. Akin to Ref. 50, we 
are localizing with respect to $\mathscr{Q}^{H}=\mathbb{Q}_{1}+\mathbb{Q}_{2}$. This corresponds to choosing the conformal Killing spinor in (36) with constant spinors $\varepsilon, \bar{\varepsilon}, \eta$, and $\bar{\eta}$ explicitly given by

$$
\begin{array}{ll}
\varepsilon=\left(\begin{array}{r}
-1 \\
0
\end{array}\right), & \bar{\varepsilon}=\left(\begin{array}{l}
1 \\
0
\end{array}\right), \\
\eta=\left(\begin{array}{l}
0 \\
1
\end{array}\right), & \bar{\eta}=\left(\begin{array}{l}
0 \\
1
\end{array}\right) .
\end{array}
$$

Then, the conformal Killing spinors are written in terms of components as follows: ${ }^{80}$

$$
\begin{array}{ll}
\xi_{1}=\left(\begin{array}{c}
-e^{-\frac{\mathrm{i}(\tau+\varphi)}{2}-\frac{y}{2 \ell}+\mathrm{i}(\zeta-\alpha) \frac{y}{\ell}} \cos \frac{\theta}{2} \\
-\mathrm{i} e^{\frac{\mathrm{i}(\tau+\varphi)}{2}-\frac{y}{2 \ell}+\mathrm{i}(\zeta-\alpha) \frac{y}{\ell}} \sin \frac{\theta}{2}
\end{array}\right), & \xi_{2}=\left(\begin{array}{c}
-\mathrm{i} e^{\frac{\mathrm{i}(\tau-\varphi)}{2}+\frac{y}{2 \ell}} \sin \frac{\theta}{2} \\
e^{\frac{\mathrm{i}(\tau-\varphi)}{2}+\frac{y}{2 \ell}} \cos \frac{\theta}{2}
\end{array}\right), \\
\bar{\xi}_{1}=\left(\begin{array}{c}
e^{-\frac{\mathrm{i} \tau}{2}+\frac{\mathrm{i} \varphi}{2}-\frac{y}{2 \ell}} \cos \frac{\theta}{2} \\
-\mathrm{i} e^{-\frac{\mathrm{i} \tau}{2}-\frac{y}{2 \ell}+\frac{i \varphi}{2}} \sin \frac{\theta}{2}
\end{array}\right), & \bar{\xi}_{2}=\left(\begin{array}{l}
\mathrm{i} e^{-\frac{\mathrm{i}(\tau+\varphi)}{2}+\frac{y}{2 \ell}-\mathrm{i}(\zeta-\alpha) \frac{y}{\ell}} \sin \frac{\theta}{2} \\
e^{-\frac{i(\tau+\varphi)}{2}+\frac{y}{2 \ell}-\mathrm{i}(\zeta-\alpha) \frac{y}{\ell}} \cos \frac{\theta}{2}
\end{array}\right),
\end{array}
$$

with their conformal cousins $\xi^{\prime}$ and $\bar{\xi}^{\prime}$ given by

$$
\begin{aligned}
& \xi_{1}^{\prime}=-\frac{1}{2 \ell}\left(\begin{array}{c}
\mathrm{i} e^{\frac{\mathrm{i}(\varphi-\tau)}{2}-\frac{y}{2 \ell}} \cos \frac{\theta}{2} \\
e^{\frac{\mathrm{i}(\varphi-\tau)}{2}-\frac{y}{2 \ell}} \sin \frac{\theta}{2}
\end{array}\right), \quad \xi_{2}^{\prime}=\frac{1}{2 \ell}\left(\begin{array}{c}
-e^{-\frac{\mathrm{i}(\tau+\varphi)}{2}+\frac{y}{2 \ell}-\mathrm{i}(\zeta-\alpha) \frac{y}{\ell}} \sin \frac{\theta}{2} \\
\mathrm{i} e^{-\frac{\mathrm{i}(\tau+\varphi)}{2}+\frac{y}{2 \ell}-\mathrm{i}(\zeta-\alpha) \frac{y}{\ell}} \cos \frac{\theta}{2}
\end{array}\right), \\
& \bar{\xi}_{1}^{\prime}=\frac{1}{2 \ell}\left(\begin{array}{c}
\mathrm{i} e^{\frac{\mathrm{i}(\tau+\varphi)}{2}-\frac{y}{2 \ell}+\mathrm{i}(\zeta-\alpha) \frac{y}{\ell}} \cos \frac{\theta}{2} \\
-e^{\frac{\mathrm{i}(x+\varphi)}{2}-\frac{y}{2 \ell}+\mathrm{i}(\zeta-\alpha) \frac{y}{\ell}} \sin \frac{\theta}{2}
\end{array}\right), \quad \bar{\xi}_{2}^{\prime}=\frac{1}{2 \ell}\left(\begin{array}{c}
e^{\frac{\mathrm{i}(\tau-\varphi)}{2}+\frac{y}{2 \ell}} \sin \frac{\theta}{2} \\
\mathrm{i} e^{\frac{\mathrm{i}(\tau-\varphi)}{2}+\frac{y}{2 \ell}} \cos \frac{\theta}{2}
\end{array}\right) .
\end{aligned}
$$

As previously mentioned, in the following, we will set

$$
\gamma=0, \quad \zeta=\frac{2 \pi M}{\beta}, \quad \text { and } \quad \alpha=\frac{2 \pi N}{\beta}
$$

with generic $M, N \in \mathbb{Z}$. This is the most general situation allowing for the full $\mathfrak{s u}(2 \mid 1)_{\ell} \oplus \mathfrak{s u}(2 \mid 1)_{r}$ to be preserved.

Finally, we will be required to introduce auxiliary spinors $\check{\xi}_{\grave{A}}$ and $\bar{\xi}_{\grave{A}}$ such that the supercharge $\mathscr{Q}^{H}$ closes off-shell for the hypermultiplets. ${ }^{81}$ Those auxiliary spinors are to satisfy

$$
\begin{aligned}
\xi_{A} \check{\xi}_{B}-\bar{\xi}_{A} \bar{\xi}_{B} & =0, & \xi^{A} \xi_{A}+\bar{\xi}^{A} \bar{\xi}_{A} & =0, \\
\bar{\xi}^{A} \bar{\xi}_{A}+\check{\xi}^{A} \check{\xi}_{A} & =0, & \xi^{A} \sigma^{m} \bar{\xi}_{A}+\check{\xi}^{A} \sigma^{m} \bar{\xi}_{A} & =0,
\end{aligned}
$$

which fixes them up to an $S L(2, \mathbb{C})$-rotation. Indeed, we may use the remaining freedom to rotate them into the simple form ${ }^{82}$

$$
\begin{aligned}
& \check{\xi}_{A}=2 \ell \xi_{A}^{\prime}, \\
& \bar{\xi}_{A}=2 \ell \bar{\xi}_{A}^{\prime} .
\end{aligned}
$$

\section{A. Vector multiplets}

The first step is to localize the vector multiplets. Here, akin to Ref. 50, a crucial and non-trivial observation is that the Yang-Mills action (39) is $\mathscr{Q}^{H}$-exact on $S^{3} \times S_{y}^{1}$-we refer to Appendix B for details. Thus, it immediately follows that the classical Yang-Mills action vanishes on the localization locus (LL), i.e.,

$$
\left.S_{\mathrm{YM}}\right|_{\mathrm{LL}}=0,
$$


and furthermore, the LL itself is simply given by

$$
F_{\mu \nu}=\phi=D_{A B}=0 \text { and } \bar{\lambda}_{A}=\lambda_{A}=0 .
$$

The latter follows from the fact that $S^{3} \times S_{y}^{1}$ has a positive scalar curvature, and thus, the Dirac operator has no zero modes; hence, there are no fermionic directions on the LL. We conclude that the LL is simply given by solutions to $F_{\mu v}=0$, that is, flat connections on $S^{3} \times S_{y}^{1}$. On $S^{3} \times S_{y}^{1}$, modulo gauge transformations, flat connections are parameterized by the holonomy around $S_{y}^{1}$, valued in the maximal torus of the gauge group $\mathbb{T} \subset G$. Therefore, the path integral over vector multiplets localizes to an integral over $\mathbb{T}$.

Some readers might find this concerning; the vector multiplets are known to produce a small $b c$ ghost system in the cohomology, ${ }^{9}$ and so one naively expects to find the fermionic zero modes on the localization locus. However, we just argued that there are none. This has a simple explanation, which (due to its importance) we will discuss in Subsection III B.

Let us proceed with the vector multiplet localization. Having dealt with the classical piece, we now turn toward computing the one-loop determinant in the background of a flat connection parameterized by the holonomy

$$
u=e^{\mathrm{i} a} \in \mathbb{T}
$$

along the circle. Using the classical action $S_{\mathrm{YM}}$ as a localizing deformation corresponds to taking the weak-coupling limit $g_{\mathrm{YM}} \rightarrow 0$. In this limit, the vector multiplet becomes a free multiplet coupled to the background flat connection $u$. The corresponding one-loop determinant is therefore the same as the one appearing in the localization computations of superconformal indices for $\mathscr{N}=2$ Lagrangian theories (in the Schur limit). More precisely-and to keep track of the more general fugacity structure-we can employ localization results for $\mathscr{N}=1$ theories on $S^{3} \times S_{y}^{1}{ }^{83,84}$ In order to be allowed to use these results, we embed our background into the one used in Ref. 84, which further requires turning on an additional background holonomy to obtain the $\mathscr{N}=2$ fugacities from the $\mathscr{N}=1$ ones. We refer to Appendix $\mathrm{C}$ for details of this procedure. The vector multiplet localization results in

$$
\frac{1}{|\mathscr{W}|} \int_{\mathbb{T}} \frac{\mathrm{d} u}{2 \pi \mathrm{i} u} \Delta_{1}(u) Z_{\mathrm{vec}}(u) \int \mathscr{D} \mathscr{H} e^{-\mathscr{S}_{\text {mat }}[\mathscr{H}, u]},
$$

where $|\mathscr{W}|$ is the order of the Weyl group, $\mathscr{D} \mathscr{H}$ denotes the path integral over the hypermultiplet fields in the background of a flat connection $u \in \mathbb{T}$, the factor $\Delta_{1}(u)$ combines the Vandermonde determinant with part of the Faddeev-Popov determinant, following Ref. 84,

$$
\Delta_{1}(u)=\prod_{\alpha \in \Delta \backslash\{0\}}\left(1-u^{\alpha}\right)=\prod_{\alpha \in \Delta_{+}}\left(1-u^{\alpha}\right)\left(1-u^{-\alpha}\right),
$$

and the one-loop determinant $Z_{\mathrm{vec}}(u)$ is given in (C50) (it can, of course, also be read off from the Schur index in Refs. 47, 48, and 83-90), ${ }^{91}$

$$
Z_{\mathrm{vec}}(u)=q^{\ell E_{0}} \mathscr{I}_{v}
$$

where

$$
\mathscr{I}_{v}=\prod_{\alpha \in \Delta}\left(q u^{\alpha} ; q\right)^{2}=(q ; q)^{2 r_{G}} \prod_{\alpha \in \Delta_{+}}\left(q u^{\alpha} ; q\right)^{2}\left(q u^{-\alpha} ; q\right)^{2}
$$

the nome is $q=e^{-\beta(1+\mathrm{i} \zeta)}$, and $E_{0}$ is the Casimir energy given by ${ }^{13,84,92,93}$

$$
\ell E_{0}=\sum_{\alpha \in \Delta^{+}}\langle\alpha, a\rangle^{2}+\frac{\operatorname{dim} G}{12} .
$$

As we will see, the first (holonomy-dependent) term in (87) will cancel in conformal theories against the similar one in the Casimir energy for the hypermultiplets. One can check that the surviving part of $E_{0}$, together with the index $\mathscr{I}_{v}$, is reproduced by the path integral of a small $b c$ ghost system [of weights $\left.\left(h_{b}, h_{c}\right)=(1,0)\right]$ on a torus $S_{\varphi}^{1} \times S_{y}^{1}$,

$$
\begin{aligned}
q^{\frac{1}{12} \operatorname{dim} G} & \prod_{\alpha \in \Delta}\left(q u^{\alpha} ; q\right)^{2} \\
& =\int \mathscr{D} b \mathscr{D} c \delta\left(\int b(\varphi, y) \mathrm{d} \varphi\right) \delta\left(\int c(\varphi, y) \mathrm{d} \varphi\right) e^{-\operatorname{Tr} \int_{S_{\varphi}^{1} \times s_{y}^{1}}^{1} \mathrm{~d} \varphi \mathrm{d} y b\left(\bar{\partial}_{w}-\mathrm{i} \frac{a}{2 \beta}\right) c} .
\end{aligned}
$$

Here, the fields $b$ and $c$ are periodic fermions on $S_{\varphi}^{1} \times S_{y}^{1}$, valued in the adjoint of the gauge group $G$ and coupled to the holonomy $a$ [cf. (82)]. The delta-functions in (88) kill modes that ought to be eliminated in a small $b c$ ghost system, known to correspond to the four-dimensional $\mathscr{N}=2$ vector multiplets. This is quite different from the ordinary $b c$ system (e.g., encountered in string theory), where one simply saturates zero modes by local insertions of $b$ and $c$ (one for each zero mode). On the contrary, for the small $b c$ ghost system, we have to perform a 
non-local operation of killing all $\varphi$-independent modes. To understand the origin of this prescription, recall that the Hilbert space $\mathscr{H}_{0}$ of the small $b c$ system is defined as a subspace of $\mathscr{H}_{b c}$ without the $c_{0}$ zero mode,

$$
\mathscr{H}_{0}=\left\{\psi \in \mathscr{H}_{b c} \mid b_{0} \psi=0\right\}
$$

The correlation functions on the torus (including the vacuum character) are then defined by

$$
\begin{aligned}
\left\langle\mathscr{O}_{1} \ldots \mathscr{O}_{n}\right\rangle & =\operatorname{Tr} \mathscr{H}_{0}\left((-1)^{F} q^{L_{0}} \widehat{\mathscr{O}}_{n}\left(\varphi_{n}, y_{n}\right) \ldots \widehat{\mathscr{O}}_{1}\left(\varphi_{1}, y_{1}\right)\right) \\
& =\operatorname{Tr} \mathscr{H}_{0}\left((-1)^{F} e^{-\left(\beta \ell-y_{n}\right) \widehat{H}} \widehat{\mathscr{O}}_{n}\left(\varphi_{n}\right) e^{-\left(y_{n}-y_{n-1}\right) \widehat{H}} \ldots e^{-\left(y_{2}-y_{1}\right) \widehat{H}} \widehat{\mathscr{O}}_{1}\left(\varphi_{1}\right) e^{-y_{1} \widehat{H}}\right),
\end{aligned}
$$

where we have assumed the ordering $0<y_{1}<y_{2}<\cdots<y_{n}<\beta \ell$, and $\widehat{H}=\frac{E}{\ell}$ generates translations in the $y$ direction. In this equation, we treat the $y$ coordinate as time, $\widehat{\mathscr{O}}_{k}\left(\varphi_{k}\right)$ are the Schrödinger picture local operators, and $\widehat{\mathscr{O}}_{k}\left(\varphi_{k}, y_{k}\right)=e^{y_{k} \widehat{H}} \widehat{\mathscr{O}}_{k}\left(\varphi_{k}\right) e^{-y_{k} \widehat{H}}$ are the Heisenberg picture local operators acting on the Hilbert space $\mathscr{H}_{0}$. The operators $\widehat{\mathscr{O}}_{k}\left(\varphi_{k}\right)$ are constructed from the modes of

$$
\begin{aligned}
& \widehat{b}(\varphi)=\sum_{m \neq 0} \widehat{b}_{m} e^{-\mathrm{i} m \varphi}, \\
& \widehat{c}(\varphi)=\sum_{m \neq 0} \widehat{c}_{m} e^{-\mathrm{i} m \varphi},
\end{aligned}
$$

which are fields of the small $b c$ system quantized on the $y=$ const. slice. Using standard arguments to obtain the path integral representation of correlators, $\widehat{b}(\varphi)$ and $\widehat{c}(\varphi)$ correspond to fermionic fields $b(\varphi, y)$ and $c(\varphi, y)$ that have no $m=0$ (i.e., $\varphi$-independent) modes in their Fourier expansions. These are precisely the modes eliminated by the delta-functions in (88). This derivation also shows that correlation functions in (88) ought to be inserted at separate "times" $y_{i}$ in order for the path integral representation to make sense. The final answer is, of course, going to be meromorphic in $w_{k}=\frac{y_{k}}{\ell}+\mathrm{i} \varphi_{k}$, which allows extending correlators outside the regime where the path integral (88) makes sense (i.e., some $y_{k}$ and $y_{n}$ may coincide in the final formula as long as $\varphi_{k}$ and $\varphi_{n}$ are distinct).

Before moving to other computations, we should add a word of caution. Note that the determinant $\Delta_{1}(u)$ can be written as

$$
\Delta_{1}(u) \sim \prod_{\alpha \in \Delta \backslash\{0\}}\langle\alpha, a\rangle \prod_{\alpha \in \Delta} \frac{\sin \frac{\langle\alpha, a\rangle}{2}}{\frac{\langle\alpha, a\rangle}{2}}=\mathscr{J}(a) \Delta_{0}(a),
$$

where $\mathscr{J}(a)$ is the usual Vandermonde and $\Delta_{0}(a)$ is a part of the Faddeev-Popov determinant that is also equal to the standard Haar measure on the Lie group. This $\Delta_{0}(a)$ is equal to the determinant of the one-dimensional analog of the $b c$ system and can provide the "missing" zero modes in the two-dimensional small $b c$ system. Therefore, we find an alternative path integral representation, at least for the partition function,

$$
Z=\frac{1}{|\mathscr{W}|} \int_{\mathbb{T}} \frac{\mathrm{d} u}{2 \pi \mathrm{i} u} \mathscr{J}(a) \int \mathscr{D}^{\prime} b \mathscr{D}^{\prime} c e^{-\operatorname{Tr} \int_{S_{\varphi}^{1} \times S_{y}^{1}} \mathrm{~d} \varphi \mathrm{d} y b\left(\bar{\partial}_{w}-\mathrm{i} \frac{a}{2 \beta}\right) c} \int \mathscr{D} \mathscr{H} e^{-\mathscr{S}_{\text {mat }}[\mathscr{H}, u]},
$$

where primes on the $b c$ measure mean that we simply drop the zero modes having $m=n=0$. This version of the $b c$ action can be interpreted as arising from the gauge fixing in two dimensions (after we localize hypermultiplets in Sec. III C), with the absence of zero modes meaning that constant gauge transformations are not summed over. However, if we start computing correlation functions with this formula, we will find that it produces the wrong Green's function for the small $b c$ system because this formula contains modes that do not act on the small $b c$ system Hilbert space $\mathscr{H}_{0}$. On the other hand, (88) gives the right propagator, as we will see.

Yet, if we use the path integral in (93) to compute correlators of operators containing only derivatives of the $c$ ghost and not $c$ itself (as appropriate in the small bc system), and furthermore only focus on gauge-invariant operators, not minding what (not gauge-invariant) Green's function $\left\langle b(0) \nabla_{w} c(w)\right\rangle$ we use in the process, we will find that (93) reproduces the correct correlation functions as well, with all the unwanted pieces canceling. Despite this, and to avoid possible confusion, we will stick to the version (88) in this work, as it manifestly describes the small $b c$ system by eliminating all the unnecessary modes.

The computation in (88) is merely a check of the statement about the small $b c$ system; essentially, we guess the action that reproduces the correct partition function and check that it indeed corresponds to the small $b c$ system. Since our localization locus does not involve gaugini zero modes, we cannot obtain the $b c$ action directly from the vector multiplet localization. Nevertheless, the two-dimensional action in (88) is the correct one to compute correlators of Schur operators containing gaugini. We now pause to elaborate on this point in more detail.

\section{B. More on the bc action}

It is well-known from Ref. 9 that certain components of the four-dimensional gaugini become fields of the small $b c$ ghost system in the chiral algebra. In fact, the computation we performed in (88) confirms this statement. In particular, for free $\mathscr{N}=2$ vector multiplets, one exactly obtains the small $b c$ ghost system. However, if we look closer at the vector multiplet-either in flat space or on $S^{3} \times S^{1}-$ and check 
how $\mathscr{Q}^{H}$ or $\mathbb{Q}_{i}$ act on the corresponding fermions, we discover the following curious fact. For example, in flat space, using notations similar to those in Ref. 9, we find

$$
\begin{array}{ll}
\left\{\mathbb{Q}_{1}, u^{A}(\bar{z}) \lambda_{A+}(z, \bar{z})\right\}=u^{A}(\bar{z}) u^{B}(\bar{z}) D_{A B}, & \left\{\mathbb{Q}_{2}, u^{A}(\bar{z}) \lambda_{A+}(z, \bar{z})\right\}=0, \\
\left\{\mathbb{Q}_{2}, u^{A}(\bar{z}) \bar{\lambda}_{A+}(z, \bar{z})\right\}=u^{A}(\bar{z}) u^{B}(\bar{z}) D_{A B}, & \left\{\mathbb{Q}_{1}, u^{A}(\bar{z}) \bar{\lambda}_{A+}(z, \bar{z})\right\}=0,
\end{array}
$$

where $u^{A}=(1, \bar{z})$. Thus, the right-hand sides are not all vanishing, and in particular, the cohomology of $\mathbb{Q}_{1}$ only contains $u^{A}(\bar{z}) \bar{\lambda}_{A+}(z, \bar{z})$, the cohomology of $\mathbb{Q}_{2}$ only contains $u^{A}(\bar{z}) \lambda_{A+}(z, \bar{z})$, and the cohomology of $\mathscr{Q}^{H}=\mathbb{Q}_{1}+\mathbb{Q}_{2}$ only contains the combination $u^{A}(\bar{z}) \lambda_{A+}(z, \bar{z})$ $-u^{A}(\bar{z}) \bar{\lambda}_{A+}(z, \bar{z})$, while the simultaneous cohomology of $\mathbb{Q}_{1}$ and $\mathbb{Q}_{2}$ appears to be empty. All these statements might look surprising because from Ref. 9 we could expect that either of these four cohomologies would give the small two-dimensional $b c$ ghost system.

Of course, we know the resolution: on-shell, auxiliary fields vanish for free vector multiplets, i.e., $D_{A B}=0$, and so the four cohomology theories mentioned above indeed coincide. Therefore, the $b c$ ghosts indeed appear in the cohomology on-shell. For interacting systems, $D_{A B}$ is no longer zero on-shell; instead, it equals the hyper-Kähler moment map describing the D-term potential for hypermultiplets, and proper identification of observables in the cohomology involves the BRST reduction procedure, as pointed out in Ref. 9. The final answer is again the same for $\mathscr{Q}^{H}, \mathbb{Q}_{i}$, or the simultaneous cohomology.

Both free and interacting systems, however, share the same property: in order to obtain the correct contribution of vector multiplets to the Q-cohomology of local operators, we need to go on-shell in the vector multiplet, i.e., integrate out auxiliary fields.

To be more precise, part of it may appear off-shell if we consider the cohomology of a single supercharge, which is what we do in the ordinary localization anyways. Note how $u^{A}(\bar{z}) \bar{\lambda}_{A+}(z, \bar{z})$ survives in the off-shell $\mathbb{Q}_{1}$ cohomology, $u^{A}(\bar{z}) \lambda_{A+}(z, \bar{z})$ belongs to the off-shell $\mathbb{Q}_{2}$ cohomology, and their difference belongs to the off-shell $\mathscr{Q}^{H}$ cohomology. This brings about another subtle point: the identification of the vector multiplet contribution as a small $b c$ system is not unique and depends on the supercharge with respect to which we compute the

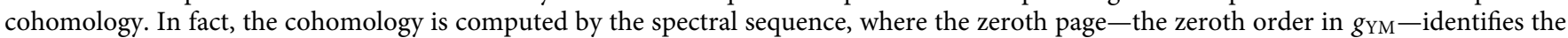
gauge and matter contributions as $\beta-\gamma$ and small $b c$ ghosts, while the exact answer is interpreted as the BRST cohomology of the zeroth page. This seems to be a universal phenomenon in gauge theories, which also takes place in two-dimensional $\mathscr{N}=(0,2)$ models ${ }^{94}[$ see also Refs. 95 and 96 for more applications in two-dimensional theories with $\mathscr{N}=(0,4)$ and $\mathscr{N}=(0,2)$ supersymmetry].

What we point out here is that the choice of the supercharge-whether it be one of the $\mathbb{Q}_{i}$ 's or $\mathscr{Q}^{H}$ - determines how we identify the small $b c$ ghosts in terms of gaugini at the zeroth page. The ghost that is found in the off-shell cohomology is always $\partial c$. Specifically, for the $\mathbb{Q}_{2}$ cohomology, we identify $\left[u^{A}(\bar{z}) \lambda_{A+}(z, \bar{z})\right]=\partial c$-which was the choice made in Ref. 9-for the $\mathbb{Q}_{1}$ cohomology, it is $\left[u^{A}(\bar{z}) \bar{\lambda}_{A+}(z, \bar{z})\right]=\partial c$, while for the $\mathscr{Q}^{H}$-cohomology, we claim $\left[u^{A}(\bar{z}) \lambda_{A+}(z, \bar{z})-u^{A}(\bar{z}) \bar{\lambda}_{A+}(z, \bar{z})\right]=\partial c$. Since these combinations are in the exact cohomology of the four-dimensional theory, they should survive the BRST reduction. The latter plays well with the fact that in the ordinary $b c$ system, one has

$$
\left\{Q_{B}, \partial c^{A}\right\} \propto f_{B C}^{A} c^{B} \partial c^{C}
$$

where $f_{B C}^{A}$ are gauge structure constants so that in the small $b c$ system, where $c$ without derivatives is thrown away, we simply have $\left\{Q_{B}, \partial c\right\}=0$.

Let us now return to the localization computation. Going on-shell is completely harmless from the vantage point of the abstract OPE approach taken in Ref. 9 but poses a fundamental challenge for the localization arguments. Indeed, in order for the localization to work, the supercharge $\mathscr{Q}^{H}$ (with respect to which we localize) must be realized off-shell, which in the standard approach only allows us to compute correlators of observables that are $\mathscr{Q}^{H}$-closed off-shell. However, it was observed in Ref. 42 that in some cases, it is possible to modify the supersymmetric localization principle to include observables that are supersymmetric on-shell. To do so, one has to supplement the localizing deformation $\exp \left(-t\left\{\mathscr{Q}^{H}, V\right\}\right)$ (with $t \rightarrow \infty$ ) by the appropriate scaling of observables that are only $\mathscr{Q}^{H}$-closed on-shell. Specifically, each insertion of $u^{A} \lambda_{A+}$ and $u^{A} \bar{\lambda}_{A+}$ should come with an extra factor of $\sqrt{t}$. This small modification makes the path-integral again independent of the parameter $t$ and thus allows for taking the localization limit $t \rightarrow \infty$. In this limit, after integrating out the bosonic fields, fermions are effectively controlled by the quadratic action. Thus, to compute correlators, one simply needs to know the Green's function and perform Wick contractions.

The latter point implies that if we can find a quadratic two-dimensional action that reproduces both the correct partition function and the expected Green's function, we have the complete answer. It turns out that the action in (88) has precisely this property; later, we will compute its Green's function, and it will match the Green's function obtained in Ref. 42 from the four-dimensional computation.

\section{Hypermultiplets and the gauged $\beta-\gamma$ system}

Let us now turn toward the localization of hypermultiplets. We expect to reproduce the $\beta-\gamma$ action from the four-dimensional hypermultiplet action evaluated on the localization locus. This type of analysis (but for a different supercharge) has first appeared in Ref. 97 for theories on $S^{4}$. In a context closer to the current paper, a similar computation in three-dimensional $\mathscr{N}=4$ theories on $S^{3}$ was performed in Ref. 50. Subsequently, it was repeated in Ref. 28 for free hypermultiplets on $S^{4}$, where it was found that they indeed localize to the $\beta-\gamma$ system on $S^{2} \subset S^{4}$. 
We expect that the resulting two-dimensional action on $S_{\varphi}^{1} \times S_{y}^{1}$ will be written in terms of fields in the $\mathscr{Q}^{H}$-cohomology of the hypermultiplet. With a certain convenient normalization, they are given by

$$
Z_{I}(\varphi, y)=\left.\sqrt{\ell} e^{-\mathrm{i} \zeta \frac{y}{2 \ell}+\mathrm{i} \alpha \frac{y}{2 \ell}+\frac{y}{2 \ell}-\mathrm{i} \frac{\varphi}{2}}\left(q_{1 I}+\mathrm{i} q_{2 I} e^{\mathrm{i} \zeta \frac{y}{\ell}-\mathrm{i} \alpha \frac{y}{\ell}-\frac{y}{\ell}+\mathrm{i} \varphi}\right)\right|_{\theta=\pi / 2},
$$

inserted anywhere on the torus located at $\theta=\pi / 2$.

In the following, we again keep $\alpha=2 \pi N / \beta$ and $\zeta=2 \pi M / \beta$ with integrals $N$ and $M$ such that the supercharge $\mathscr{Q}^{H}$ is conserved. Furthermore, we close our supercharge $\mathscr{Q}^{H}$ off-shell: the hypermultiplet action (40) preserves off-shell supersymmetry $\mathscr{Q}^{H}$, given that the equations for the auxiliary spinors $\breve{\xi}_{A}$ and $\bar{\xi}_{A}$ in (77) are satisfied; the solutions are given in (78) and (79). It is straightforward to check the off-shell closure of $\mathscr{Q}^{H}$, i.e., the hypermultiplet supersymmetry transformations (44) square into a sum of bosonic symmetries off-shell, ${ }^{66}$

$$
\begin{aligned}
Q^{2} q_{A} & =\mathrm{i} v^{m} D_{m} q_{A}+\mathrm{i} \Phi q_{A}+w q_{A}+\Theta_{A B} q^{B}, \\
Q^{2} \psi & =\mathrm{i} v^{m} D_{m} \psi+\mathrm{i} \Phi \psi+\frac{3}{2} w \psi-\Theta \psi+\frac{\mathrm{i}}{4} \sigma^{k l} \psi D_{k} v_{l}, \\
Q^{2} \bar{\psi} & =\mathrm{i} v^{m} D_{m} \bar{\psi}+\mathrm{i} \Phi \bar{\psi}+\frac{3}{2} w \bar{\psi}+\Theta \psi+\frac{\mathrm{i}}{4} \bar{\sigma}^{k l} \bar{\psi} D_{k} v_{l}, \\
Q^{2} F_{A} & =\mathrm{i} v^{m} D_{m} F_{A}+\mathrm{i} \Phi F_{A}+2 w F_{A}+\check{\Theta}_{A B} F^{B},
\end{aligned}
$$

where for $Q=\mathscr{Q}^{H}$, we explicitly find that

$$
\begin{aligned}
v_{m} \mathrm{~d} x^{m} & =4 \mathrm{i} \cos ^{2} \theta\left(\ell \mathrm{d} \tau+\frac{\zeta}{\ell} \mathrm{d} y\right), & w & =0, \\
\Phi & =4 \mathrm{i} \cos \theta e^{-\mathrm{i} \tau-\mathrm{i} \zeta \frac{y}{\ell}-\mathrm{i} \alpha \frac{y}{\ell}}\left(\phi e^{2 \mathrm{i} \alpha \frac{y}{\ell}}-\bar{\phi} e^{2 \mathrm{i} \mathrm{i}+2 \mathrm{i} \zeta \frac{y}{\ell}}\right), & \Theta & =\frac{2 \mathrm{i}}{\ell}, \\
\check{\Theta}_{A B} & =-\frac{4 \alpha}{\ell}\left(\begin{array}{cc}
\mathrm{i} e^{\mathrm{i} \zeta \frac{y}{\ell}-\mathrm{i} \alpha \frac{y}{\ell}-\frac{y}{\ell}+\mathrm{i} \varphi} \sin \theta & 1 \\
1 & -\mathrm{i} e^{\mathrm{i} \alpha \frac{y}{\ell}-\mathrm{i} \zeta \frac{y}{\ell}+\frac{y}{\ell}-\mathrm{i} \varphi} \sin \theta
\end{array}\right), & \Theta_{A B} & =0 .
\end{aligned}
$$

To localize the hypermultiplets, we use the canonical localizing deformation $\mathscr{Q}^{H} V$, where

$$
V=\sum_{\alpha, I} \psi_{\alpha I}\left(\mathscr{Q}^{H} \psi_{\alpha I}\right)^{*}+\sum_{\dot{\alpha}, I} \bar{\psi}_{I}^{\dot{\alpha}}\left(\mathscr{Q}^{H} \bar{\psi}_{I}^{\dot{\alpha}}\right)^{*}
$$

It has no fermionic zero modes (which is expected because the $\mathscr{Q}^{H}$-cohomology of a hypermultiplet is purely bosonic). The bosonic zero modes, i.e., the localization locus, are determined by the BPS equations $\mathscr{Q}^{H} \psi=\mathscr{Q}^{H} \bar{\psi}=0$, which explicitly read

$$
\begin{aligned}
& 0=2 \sigma^{\mu} \bar{\xi}_{A} \mathscr{D}_{\mu} q_{I}^{A}+\sigma^{\mu} \mathscr{D}_{\mu} \bar{\xi}_{A} q_{I}^{A}-4 \mathrm{i} \xi_{A} \bar{\phi}_{{ }_{I}}^{A}{ }_{I}+2 \check{\xi}_{A} F_{I}^{A}, \\
& 0=2 \bar{\sigma}^{\mu} \xi_{A} \mathscr{D}_{\mu} q_{I}^{A}+\bar{\sigma}^{\mu} \mathscr{D}_{\mu} \xi_{A} q_{I}^{A}-4 \mathrm{i} \bar{\xi}_{A} \phi q_{I}^{A}{ }_{I}+2 \bar{\xi}_{A} F_{I}^{A},
\end{aligned}
$$

where the covariant derivatives act on the hypermultiplet scalars as follows:

$$
\mathscr{D}_{\mu} q_{A I}=\partial_{\mu} q_{A I}-\mathrm{i}\left(A_{\mu}\right)_{I}^{I} q_{A J}+\mathrm{i} V_{A}^{B} q_{B I},
$$

with $V_{B}^{A}$ being the $S U(2)_{R}$ background gauge field and $\left(A_{\mu}\right)_{I}{ }^{J}$ being a dynamical gauge field in the vector multiplet (of course, one can also couple to background vector multiplet, which then induces a "flavor-fugacity" and/or mass for the hypermultiplet).

For our purposes, it is enough to localize hypermultiplets in a background of localized vector multiplets. From Subsection III A, we know that all the vector multiplet fields vanish in such a background, except for the component $A_{y}$ that takes on constant values parameterizing flat connections on $S^{3} \times S_{y}^{1}$. At this point of the computation, there is no distinction between the gauge and flavor background holonomies, but, of course, at the end, we will integrate over the former but not over the latter. Hence, with the normalization as in (82), we have

$$
A=\frac{a}{\beta \ell} \mathrm{d} y \quad \text { with } \quad a \in \mathfrak{t}=\text { Lie } \mathbb{T} .
$$


Furthermore, due to $\alpha$ being non-zero, there is a non-vanishing R-symmetry holonomy, given by

$$
V_{A}^{B}=\frac{\alpha}{2 \ell} \mathrm{d} y\left(\tau_{3}\right)_{A}^{B} \quad \text { with } \quad \alpha=\frac{2 \pi N}{\beta} .
$$

Note that only the $\mathscr{D} y$ component of the covariant derivative in (105) acting on $q_{A I}$ involves such holonomies, while the other derivatives can be (and are) replaced by the usual partial derivative $\partial_{\mu}$ in what follows. We can solve the BPS-equations for the auxiliary fields and upon substituting back into the equations, we obtain

$$
\partial_{\tau} q_{A I}=0
$$

which effectively compactifies fields in the $\tau$ direction: $\tau$-independent configurations are described by fields on $D^{2} \times S_{y}^{1}$, where $D^{2}$ is parameterized by $(\theta, \varphi)$.

Now, to proceed, we impose the reality conditions on $F$ and $q$, as given in (45). With these conditions, the remaining BPS equations become

$$
\begin{aligned}
& \left(\frac{1}{\cos \theta} \partial_{\theta}-\frac{\mathrm{i}}{\sin \theta} \partial_{\varphi}\right)\left(e^{+\frac{y}{2 \ell}} q_{1 I}\right)=\mathrm{i} e^{\mathrm{i} \varphi+\frac{\mathrm{i}(\zeta-\alpha) y}{\ell}}\left(\ell \mathscr{D}_{y}-\zeta \partial_{\varphi}\right)\left(e^{-\frac{y}{2 \ell}} q_{2 I}\right), \\
& \left(\frac{1}{\cos \theta} \partial_{\theta}+\frac{\mathrm{i}}{\sin \theta} \partial_{\varphi}\right)\left(e^{-\frac{y}{2 \ell}} q_{2 I}\right)=\mathrm{i} e^{-\mathrm{i} \varphi-\frac{\mathrm{i}(\zeta-\alpha) y}{\ell}}\left(\ell \mathscr{D}_{y}-\zeta \partial_{\varphi}\right)\left(e^{\frac{y}{2 \ell}} q_{1 I}\right) .
\end{aligned}
$$

Additionally, we get expressions for the auxiliary fields $F_{A I}$ in terms of $q_{A I}$ given as solutions of the partial differential equations (109) and (110),

$$
F_{A I}=\frac{\mathrm{i}}{2 \ell}\left(2 \tan \theta \partial_{\theta}+1\right) q_{A I}
$$

To simplify (109) and (110), it is useful to introduce complex coordinates $(v, \bar{v})$ on the $(\theta, \varphi)$-disk, which undergo a twist as we move along the $y$ direction,

$$
v=\sin \theta e^{\mathrm{i}\left(\varphi+\zeta \frac{y}{\ell}\right)}
$$

In terms of this coordinate, the BPS equations take the more suggestive form

$$
\begin{aligned}
& \partial_{\nu}\left(e^{+\frac{y}{2 \ell}} q_{1 I}\right)=\frac{\mathrm{i}}{2} e^{-\frac{\mathrm{i} \alpha y}{\ell}} \ell \mathscr{D}_{y}\left(e^{-\frac{y}{2 \ell}} q_{2 I}\right), \\
& \partial_{\bar{v}}\left(e^{-\frac{y}{2 \ell}} q_{2 I}\right)=\frac{\mathrm{i}}{2} e^{\frac{\mathrm{i} \alpha y}{\ell}} \ell \mathscr{D}_{y}\left(e^{\frac{y}{2 \ell}} q_{1 I}\right) .
\end{aligned}
$$

To solve these equations, let us expand the fields $q_{A I}$ in terms of Fourier modes of $y$ (taking into account the twisted sector periodicity),

$$
\begin{aligned}
q_{1 I} & =\sum_{n \in \mathbb{Z}} a_{I, n}^{+}(v, \bar{v}) e^{\frac{2 \pi i n y}{\beta \ell}-\frac{y}{2 \ell}}, \\
\mathrm{i} q_{2 I} & =\sum_{n \in \mathbb{Z}} a_{I, n}^{-}(v, \bar{v}) e^{\frac{2 \pi i n y}{\beta \ell}+\frac{y}{2 \ell}} .
\end{aligned}
$$

This separates the variables $v, \bar{v}$ from $y$, and including the background gauge holonomy $a$ and the R-symmetry holonomy $\alpha=2 \pi N / \beta$, we obtain the following equations for the modes:

$$
\begin{aligned}
\partial_{\nu} a_{I, n}^{+} & =+\frac{\mathrm{i}}{2}\left(\frac{2 \pi(n+N)}{\beta}-\frac{a}{\beta}\right) a_{I, n+N}^{-}, \\
\partial_{\bar{v}} a_{I, n+N} & =-\frac{\mathrm{i}}{2}\left(\frac{2 \pi n}{\beta}-\frac{a}{\beta}\right) a_{I, n}^{+} .
\end{aligned}
$$

For ease of notation, let us define

$$
\sigma_{n} \equiv \frac{2 \pi n}{\beta}-\frac{a}{\beta} .
$$


The solutions to these equations that are regular on the disk $D^{2}$ take the following form:

$$
\begin{aligned}
a_{I, n}^{+}(v, \bar{v}) & =\sum_{m \in \mathbb{Z}} \mathrm{i} x_{I}^{m, n} \sqrt{\frac{\sigma_{n+N}}{\sigma_{n}}} I_{m}\left(\sqrt{\sigma_{n} \sigma_{n+N}} \sin \theta\right) e^{\mathrm{i} m\left(\varphi+\zeta \frac{y}{\ell}\right),} \\
a_{I, n+N}^{-}(v, \bar{v}) & =\sum_{m \in \mathbb{Z}} x_{I}^{m+1, n} I_{m}\left(\sqrt{\sigma_{n} \sigma_{n+N}} \sin \theta\right) e^{\mathrm{i} m\left(\varphi+\zeta \frac{y}{\ell}\right)},
\end{aligned}
$$

where $I_{m}$ are modified Bessel functions and $x_{I}^{m, n}$ are complex constants. Inserting them back into the Fourier series, we find the following most general smooth solution on $D^{2} \times S_{y}^{1}$ in the form of a double Fourier series on $S_{\varphi}^{1} \times S_{y}^{1}$,

$$
\begin{aligned}
q_{1 I} & =\sum_{n, m \in \mathbb{Z}} \mathrm{i} x_{I}^{m, n} \sqrt{\frac{\sigma_{n+N}}{\sigma_{n}}} I_{m}\left(\sqrt{\sigma_{n} \sigma_{n+N}} \sin \theta\right) e^{\mathrm{i} m\left(\varphi+\zeta \frac{y}{\ell}\right)+\frac{2 \pi i n y}{\beta \ell}-\frac{y}{2 \ell}}, \\
\mathrm{i} q_{2 I} & =\sum_{n, m \in \mathbb{Z}} x_{I}^{m+1, n-N} I_{m}\left(\sqrt{\sigma_{n-N} \sigma_{n}} \sin \theta\right) e^{\mathrm{i} m\left(\varphi+\zeta \frac{y}{\ell}\right)+\frac{2 \pi i n y}{\beta \ell}+\frac{y}{2 \ell}} .
\end{aligned}
$$

We can plug this into the definition of the two-dimensional fields (96) to find that at the boundary $\theta=\pi / 2$ of $D^{2} \times S_{y}^{1}$, we obtain

$$
\begin{gathered}
Z_{I}(\varphi, y)=\sqrt{\ell} e^{-\frac{\mathrm{i}(\zeta-\alpha) y}{2 \ell}-\frac{\mathrm{i}}{2} \varphi} \sum_{n, m \in \mathbb{Z}} x_{I}^{m, n}\left(I_{m-1}\left(\sqrt{\sigma_{n} \sigma_{n+N}}\right)+\mathrm{i} \sqrt{\frac{\sigma_{n+N}}{\sigma_{n}}} I_{m}\left(\sqrt{\sigma_{n} \sigma_{n+N}}\right)\right) \\
\times e^{\mathrm{i} m\left(\varphi+\zeta \frac{y}{\ell}\right)+\frac{2 \pi n i n y}{\beta \ell}} .
\end{gathered}
$$

This expression immediately implies that all the modes $x_{I}^{m, n}$ are encoded in the two-dimensional fields $Z_{I}$ living on $S_{\varphi}^{1} \times S_{y}^{1}$. In other words, regular solutions to the BPS equations-i.e., the localization locus-are indeed parameterized by fields on the torus (symplectic bosons, as we will see soon). The above equation also shows that the symplectic bosons $Z_{I}$ are naturally anti-periodic in the $\varphi$ direction, while the periodicity in the $y$ direction is determined by $\zeta-\alpha=2 \pi(M-N) / \beta$ : they are periodic for $M-N \equiv 0 \bmod 2$ and anti-periodic for $M-N \equiv 1 \bmod 2$.

Now, let us turn to the hypermultiplet action (40) and evaluate it on the localization locus. At the saddle point, we have the following bosonic action:

$$
\left.\mathscr{S}_{\text {mat }}\right|_{\text {BPS }}=\int_{S^{3} \times S^{1}}\left[\frac{1}{2} \mathscr{D}_{\mu} q^{A I} \mathscr{D}^{\mu} q_{A I}+\frac{1}{2 \ell^{2}} q^{A I} q_{A I}-\frac{1}{2} F^{A I} F_{A I}\right] \operatorname{vol}_{S^{3} \times S^{1}},
$$

where we integrate over the whole spacetime $S^{3} \times S^{1}$, with $\operatorname{vol}_{S^{3} \times S^{1}}=\ell^{3} \sin \theta \cos \theta \mathrm{d} \theta \mathrm{d} \varphi \mathrm{d} \tau \mathrm{d} y$. Now, using $\tau$-invariance and the expression for $F_{A I}$, we can rewrite this as

$$
\left.\mathscr{S}_{\text {mat }}\right|_{\text {BPS }}=-\left[\int_{0}^{2 \pi} \ell \mathrm{d} \tau\right] \int_{S_{y}^{1} \times D^{2}}\left(\nabla_{a} \Phi^{a}\right) \operatorname{vol}_{S_{y}^{1} \times D^{2}}
$$

where we defined

$$
\Phi^{a}=\frac{1}{\sqrt{S \bar{S}}} \varepsilon^{a b c d}\left(\xi^{A} \sigma_{b} \bar{\xi}_{A}\right)\left(\xi_{B} \sigma_{c} \bar{\xi}_{C}\right) q_{I}^{B} \mathscr{D}_{d} q^{C I}-2 \mathrm{i} \sqrt{\frac{\bar{S}}{\bar{S}}}\left(\xi^{\prime}{ }_{A} \sigma^{a} \bar{\xi}_{B}\right) q^{A}{ }_{I} q^{B I}
$$

and $S_{y}^{1} \times D^{2}$ is the space parameterized by $(y, \theta, \varphi)$, with $\operatorname{vol}_{S_{y}^{1} \times D^{2}}=\ell^{2} \sin \theta \mathrm{d} y \mathrm{~d} \theta \mathrm{d} \varphi$. Furthermore, we defined the quantities $S$ and $\bar{S}$ as follows:

$$
S=\xi^{A} \xi_{A}=-2 e^{\mathrm{i} \tau} \cos \theta, \quad \bar{S}=\bar{\xi}^{A} \bar{\xi}_{A}=-2 e^{-\mathrm{i} \tau} \cos \theta
$$

Hence, we apply Stokes's theorem and find that the now three-dimensional action further reduces to a two-dimensional action on the boundary torus $\partial\left(S_{y}^{1} \times D^{2}\right)=S_{y}^{1} \times S_{\varphi}^{1}$,

$$
\left.\mathscr{S}_{\text {mat }}\right|_{\text {BPS }}=-2 \pi \ell^{2} \int_{S_{y} \times S_{\varphi}^{1}} \Phi \cdot \mathrm{d} S,
$$

where $\mathrm{d} S$ is along the normal (i.e., along the frame $e_{1}=\frac{1}{\ell} \frac{\partial}{\partial \theta}$ ) of the surface $S_{y}^{1} \times S_{\varphi}^{1}$. We now define the elementary fields $Z_{I}$ in the $\mathscr{Q}^{H}$ cohomology as in (96). With this definition, we can write the remaining action on the torus as

$$
\begin{aligned}
\left.\mathscr{S}_{\text {mat }}\right|_{\text {BPS }} & =4 \pi \mathrm{i} \int_{S_{y}^{1} \times S_{\varphi}^{1}} \mathrm{~d} \varphi \mathrm{d} y \varepsilon^{I J} Z_{I} \nabla_{\bar{w}} Z_{J} \\
& =4 \pi \mathrm{i} \ell \int_{S_{y}^{1} \times S_{\varphi}^{1}} \mathrm{~d}^{2} w \mathcal{E}^{I I} Z_{I} \nabla_{\bar{w}} Z_{J},
\end{aligned}
$$


where

$$
\nabla_{\bar{w}}=\frac{\mathrm{i}}{2}(1+\mathrm{i} \zeta) \partial_{\varphi}+\frac{\ell}{2} \mathscr{D}_{y}=\partial_{\bar{w}}-\mathrm{i} \frac{a}{2 \beta},
$$

and we used the complex coordinates $(w, \bar{w})$ on the torus previously introduced in (67),

$$
w=\frac{y}{\ell}+\mathrm{i}\left(\varphi+\zeta \frac{y}{\ell}\right), \quad \bar{w}=\frac{y}{\ell}-\mathrm{i}\left(\varphi+\zeta \frac{y}{\ell}\right) .
$$

Note that the induced two-dimensional metric on $S_{y}^{1} \times S_{\varphi}^{1}$ is given by

$$
\mathrm{d} s_{2}^{2}=\ell^{2}\left[\mathrm{~d} \phi^{2}+\frac{2 \zeta}{\ell} \mathrm{d} \phi \mathrm{d} y+\frac{1}{\ell^{2}}\left(1+\zeta^{2}\right) \mathrm{d} y^{2}\right]=\ell^{2} \mathrm{~d} w \mathrm{~d} \bar{w}
$$

where $\zeta$ can be identified as the complex structure parameter of the torus.

If we replace $S_{y}^{1}$ by a line, $w, \bar{w}$ turn into coordinates on the cylinder and-as previously mentioned-we can conformally map it to the plane via (assume $\zeta=0$ )

$$
z=e^{w}, \quad \bar{z}=e^{\bar{w}}
$$

and so the fields transform as

$$
Z_{I}=\sqrt{z Z_{I}}
$$

and the action (assuming $a=0$ ) becomes $\propto \int \mathrm{d}^{2} z \varepsilon^{I I} \widehat{Z}_{I} \partial_{\bar{z}} \widehat{Z}_{J}$, i.e., the symplectic boson on the plane. In this paper, however, we are mostly interested in the case when the two-dimensional theory lives on the torus.

The symplectic boson theory is, of course, a special case of the $\beta-\gamma$ system, where both fields are of conformal dimension $1 / 2$. Separating $Z_{I}$ into $\gamma$ and $\beta$ requires a choice of Lagrangian splitting of the representation space of half-hypermultiplets. In general, there is no preferred one. However, in the case of full hypermultiplets, the representation of half-hypermultiplets is of the form $\mathbf{R} \cong \mathscr{R} \oplus \overline{\mathscr{R}}$, where $Z_{1} \in \mathscr{R}$ and $Z_{2} \in \overline{\mathscr{R}}$. In this case, we can simply choose

$$
\gamma=Z_{1}, \quad \beta=Z_{2},
$$

and the action becomes $8 \pi \mathrm{i} \ell \int \mathrm{d}^{2} w \beta \nabla_{\bar{w}} \gamma$, i.e., the standard action of the $\beta-\gamma$ system. However, because general superconformal field theories may also contain half-hypermultiplets, we will keep the action in the more general form of Eq. (130).

\section{One-loop determinant}

To complete the localization, we now need to determine the one-loop determinant $Z_{\text {mat }}$ for small fluctuations around the BPS locus. In general, the one-loop determinant might depend on all parameters of the localization locus, which, in our case, are the holonomy $u$ and the symplectic bosons $Z_{I}(w, \bar{w})$. As it is easy to see in our problem, the quadratic action for small fluctuations of the hypermultiplet fields around the LL actually does not depend on $Z_{I}(w, \bar{w})$. Unlike the vector multiplet case, the supersymmetry variation $\mathscr{Q}^{H} \psi$ is linear in the hypermultiplet fields, and hence, the canonical localizing term $\left|\mathscr{Q}^{H} \psi\right|^{2}+\ldots$ is purely quadratic in the hypermultiplet fields, implying that the one-loop determinant can only depend on $u$.

To find $Z_{\text {mat }}(u)$, we are going to utilize the fact that the four-dimensional hypermultiplet action is already quadratic after localizing the vector multiplet part. Therefore, we can compute the four-dimensional hypermultiplet path integral exactly and equate it with the localization computation result for the hypermultiplet. In other words, we have the following relation:

$$
Z_{\text {mat }}(u) \int \mathscr{D} Z_{I} e^{-4 \pi \mathrm{i} \ell \int_{S_{y}^{1} \times S_{\varphi}^{1}} \mathrm{~d}^{2} w \varepsilon^{I I} Z_{I} \nabla_{\bar{w}} Z_{J}}=\int \mathscr{D} \mathscr{H} e^{-\mathscr{S}_{\text {mat }}[\mathscr{H}, u]}
$$

Hence, to find $Z_{\text {mat }}(u)$, we simply have to compute the quadratic path integrals on the two sides of the equality and take their ratio.

The right-hand side of (137) describes a free hypermultiplet on $S^{3} \times S_{y}^{1}$ coupled to the background $G$-holonomy $u$ and a possible $(R+r)$ holonomy $\alpha=2 \pi N / \beta$. For $N=0 \bmod 2$, this is nothing but the flavored Schur index of a free hypermultiplet. Again referring to Appendix C for a review of localization results in four dimensions, it is given by

$$
\begin{aligned}
& N=0 \bmod 2: \\
& (137)=q^{\frac{\operatorname{dim} \mathbf{R}}{48}-\frac{1}{4} \sum_{w \in \mathbf{R}}\langle w, a\rangle^{2}} \prod_{w \in \mathbf{R}} \frac{1}{\left[\left(\sqrt{q} u^{w} ; q\right)\left(\sqrt{q} u^{-w} ; q\right)\right]^{1 / 2}}, \quad q=e^{-\beta(1+\mathrm{i} \zeta)},
\end{aligned}
$$


where $\mathbf{R}$ is the total representation of half-hypermultiplets (including the full hypermultiplets). With $N=1 \bmod 2$, we have an extra insertion of $(-1)^{2(R+r)}$, which does not affect the Casimir energy but slightly modifies the Schur index of a hypermultiplet. Specifically, the "single-letters" have $(-1)^{2(R+r)}=-1$, and so their contributions become $-\sqrt{q}$ instead of the usual $\sqrt{q}$. Thus, the answer reads

$$
\begin{aligned}
& N=1 \bmod 2: \\
& (137)=q^{\frac{\operatorname{dim} \mathbf{R}}{48}-\frac{1}{4} \sum_{w \in \mathbf{R}}\langle w, a\rangle^{2}} \prod_{w \in \mathbf{R}} \frac{1}{\left[\left(-\sqrt{q} u^{w} ; q\right)\left(-\sqrt{q} u^{-w} ; q\right)\right]^{1 / 2}},
\end{aligned}
$$

with $q=e^{-\beta(1+\mathrm{i} \zeta)}$.

On the other hand, the two-dimensional symplectic boson path integral on $S_{\varphi}^{1} \times S_{y}^{1}$-anti-periodic in $\varphi$ and either periodic or antiperiodic in $y$-can be easily evaluated,

$$
\begin{aligned}
& \text { Periodic in } y \text { : } \\
& \int \mathscr{D} Z_{I} e^{-4 \pi \mathrm{i} \ell \int_{S_{y} \times S_{\varphi}^{1}} \mathrm{~d}^{2} w w^{I I} Z_{I} \nabla_{\bar{w}} Z_{J}}=q^{\frac{\operatorname{dim} \mathbf{R}}{48}} \prod_{w \in \mathbf{R}} \frac{1}{\left[\left(\sqrt{q} u^{w} ; q\right)\left(\sqrt{q} u^{-w} ; q\right)\right]^{1 / 2}}, \\
& \text { Anti - periodic in } y \text { : } \\
& \int \mathscr{D} Z_{I} e^{-4 \pi i \ell \int_{s_{y} \times S_{\varphi}} \mathrm{d}^{2} w \varepsilon^{I J} Z_{I} \nabla_{\bar{w}} Z_{J}}=q^{\frac{\operatorname{dim} \mathbf{R}}{48}} \prod_{w \in \mathbf{R}} \frac{1}{\left[\left(-\sqrt{q} u^{w} ; q\right)\left(-\sqrt{q} u^{-w} ; q\right)\right]^{1 / 2}} .
\end{aligned}
$$

Matching this to the two expressions for Schur indices given above implies that $Z_{\mathrm{mat}}(u)$ only receives contributions from the difference of Casimir energies in four and two dimensions,

$$
Z_{\text {mat }}(u)=q^{-\frac{1}{4} \sum_{w \in R}\langle w, a\rangle^{2}} .
$$

For a superconformal field theory (without flavor fugacities), this part of the Casimir term cancels against the similar one in the vector multiplet path integral. Specifically, the $a$-dependent piece in (87) (which also did not cancel there) combines with what we have found here into

$$
\sum_{\alpha \in \Delta^{+}}\langle\alpha, a\rangle^{2}-\frac{1}{4} \sum_{w \in \mathbf{R}}\langle w, a\rangle^{2}=\frac{1}{2}\left(C(\mathbf{a d j})-\frac{1}{2} C(\mathbf{R})\right) \operatorname{Tr} a^{2},
$$

where $C(\ldots)$ denotes the index of the corresponding representation. The expression in parentheses is precisely the coefficient of the betafunction (for each simple gauge factor $G_{i}$ ) that vanishes in the superconformal field theory. One can also check that for each $G_{i}$,

$$
2 C(\mathbf{a d j})-C(\mathbf{R})=2 h^{\vee}\left(G_{i}\right)-\sum_{x} C\left(\mathbf{R}_{i, x}\right) \prod_{j \neq i} \operatorname{dim} \mathbf{R}_{j, x}-\sum_{y} 2 C\left(\mathscr{R}_{i, y}\right) \prod_{j \neq i} \operatorname{dim} \mathscr{R}_{j, y}=0,
$$

according to (46).

Hence, in the end, we find that in the absence of flavor fugacities, the one-loop determinants do not produce any additional factors, and the four-dimensional theory localizes precisely to the two-dimensional theory of gauged symplectic bosons,

$$
\begin{aligned}
& \int \mathscr{D} \mathscr{V} \mathscr{D} \mathscr{H} e^{-\mathscr{S}[\mathscr{V}, \mathscr{C}]}= \\
& \quad \frac{1}{|\mathscr{W}|} \int_{\mathbb{T}} \frac{\mathrm{d} u}{2 \pi \mathrm{i} u} \Delta_{1}(u) \int \mathscr{D} Z_{I} \mathscr{D} b \mathscr{D} c \delta\left(\int b(\varphi, y) \mathrm{d} \varphi\right) \delta\left(\int c(\varphi, y) \mathrm{d} \varphi\right) e^{-\mathscr{S}_{2 \mathrm{~d}}},
\end{aligned}
$$

where $\mathscr{V}$ and $\mathscr{H}$ denote the four-dimensional vector and hypermultiplets, respectively, and with the two-dimensional action given by

$$
\mathscr{S}_{2 \mathrm{~d}}=\operatorname{Tr} \int_{S_{y}^{1} \times S_{\varphi}^{1}} \mathrm{~d} y \mathrm{~d} \varphi b\left(\bar{\partial}_{w}-\mathrm{i} \frac{a}{2 \beta}\right) c+4 \pi \mathrm{i} \int_{S_{y}^{1} \times S_{\varphi}^{1}} \mathrm{~d} y \mathrm{~d} \varphi \varepsilon^{I J} Z_{I} \nabla_{\bar{w}} Z_{J}
$$

with

$$
\nabla_{\bar{w}}=\partial_{\bar{w}}-\mathrm{i} \frac{a}{2 \beta},
$$

and where one integrates $u=e^{\mathrm{i} a}$ over the maximal torus $\mathbb{T} \subset G$. Furthermore, changing periodicity of symplectic bosons along $S_{y}^{1}$ corresponds to the insertion of $(-1)^{2(R+r)}$ in the Schur index. 
If we turn on flavor fugacities, i.e., treat some components of $a$ as vevs $a_{f}$ of the background vector multiplets, then $Z_{\text {mat }}(u)$ does not fully cancel against the vector multiplet contribution. In this case, there is a leftover factor producing a mismatch between the left- and right-hand sides of (145) given by the "flavor Casimir term,"

$$
Z_{\text {Cas }}^{\mathrm{f}}\left(q, a_{f}\right)=q^{-\frac{1}{4} \sum_{w_{f} \in \mathbf{R}_{f}}\left\langle w_{f}, a_{f}\right\rangle^{2}}
$$

where the summation is over weights of the flavor group representation.

The correlation functions of general BRST-closed observables (built from the $Z_{I}$ and $b c$ ghosts) can now be computed using the twodimensional Gaussian path integral (145) and (146), which is the final output of our localization computation in the absence of surface defects. They capture the full chiral algebra of the four-dimensional $\mathscr{N}=2$ superconformal field theory.

Let us now also briefly discuss the Casimir energy in the presence of the deformations in terms of the integers $M$ and $N$. The way we have introduced those deformations as special values of the more general fugacities $\zeta$ and $\alpha$ [see Eq. (48)], one would naively expect that the corresponding Casimir energy is simply obtained by evaluating the expressions of the $\mathscr{N}=2$ Casimir energy in terms of $\zeta$ and $\alpha$ in Eq. (C48) at those special values. However, recalling the definition of the (scheme-independent) supersymmetric Casimir energy as ${ }^{93}$

$$
E_{\text {susy }}=-\lim _{\beta \rightarrow \infty} \frac{\mathrm{d}}{\mathrm{d} \beta} Z_{S^{3} \times S_{\beta}^{1}}
$$

we observe that $M$ and $N$ cannot contribute to it, as the special values of $\zeta$ and $\alpha$ in Eq. (48) are explicitly $\beta$-dependent.

Alternatively, we may apply a result of Ref. 89 , which relates the supersymmetric Casimir energy, ${ }^{9}$

$$
E_{\text {susy }}=\left\langle H_{\text {susy }}\right\rangle \text {, }
$$

where $H_{\text {susy }}$ is the supersymmetric Hamiltonian in the one-dimensional quantum mechanics, for $\mathscr{N}=1$ theories on $S^{3} \times S^{1}$, to a special limit of the "single letters" of the index, where one explicitly employs the "supersymmetric" regularization scheme. However, as discussed above, the Schur index and thus its single-letters are unaffected by the inclusion of $M$ and $N$ and thus neither is the Casimir energy.

\section{Inclusion of the R-symmetry defect}

Let us now see how the analysis changes in the presence of a surface defect that creates the $(-1)^{2(R+r)}$ monodromy. Recall that the defect is located at $\theta=0$, stretching in the $\tau$ and $y$ directions, making all the hypermultiplet fields anti-periodic in the $\varphi$ coordinate. All the analyses above up to (and including) Eqs. (117) and (118) hold without changes. The next step-the Fourier expansion of $a_{I, n}^{ \pm}$as a function of $\varphi$-is modified since now we have to expand in half-integral modes. As a result, the general solution, if we require $q_{11} \sim \theta^{1 / 2}$ and $q_{21} \sim \theta^{-1 / 2}$ at $\theta=0$, is

$$
\begin{aligned}
a_{I, n}^{+}(v, \bar{v})= & \sum_{m \in \mathbb{Z}} \mathrm{i} x_{I}^{m, n} \sqrt{\frac{\sigma_{n+N}}{\sigma_{n}}} I_{\left|m+\frac{1}{2}\right|}\left(\sqrt{\sigma_{n} \sigma_{n+N}} \sin \theta\right) e^{\mathrm{i}\left(m+\frac{1}{2}\right)\left(\varphi+\zeta \frac{y}{\ell}\right)}, \\
a_{I, n+N}^{-}(v, \bar{v})= & x_{I}^{0, n} I_{-\frac{1}{2}}\left(\sqrt{\sigma_{n} \sigma_{n+N}} \sin \theta\right) e^{-\frac{\mathrm{i}}{2}\left(\varphi+\zeta \frac{y}{\ell}\right)} \\
& +\sum_{\substack{m \in \mathbb{Z} \\
m \neq 0}} x_{I}^{m, n} I_{\left|m-\frac{1}{2}\right|}\left(\sqrt{\sigma_{n} \sigma_{n+N}} \sin \theta\right) e^{\mathrm{i}\left(m-\frac{1}{2}\right)\left(\varphi+\zeta \frac{y}{\ell}\right)} .
\end{aligned}
$$

Similarly, if we require $q_{11} \sim \theta^{-1 / 2}$ and $q_{21} \sim \theta^{1 / 2}$ at $\theta=0$, we find the following solution:

$$
\begin{aligned}
a_{I, n}^{+}(v, \bar{v})= & \mathrm{i} x_{I}^{0, n} \sqrt{\frac{\sigma_{n+N}}{\sigma_{n}}} I_{-\frac{1}{2}}\left(\sqrt{\sigma_{n} \sigma_{n+N}} \sin \theta\right) e^{\frac{\mathrm{i}}{2}\left(\varphi+\zeta \frac{y}{\ell}\right)} \\
& +\sum_{\substack{m \in \mathbb{Z} \\
m \neq 0}} \mathrm{i} x_{I}^{m, n} \sqrt{\frac{\sigma_{n+N}}{\sigma_{n}}} I_{\left|m+\frac{1}{2}\right|}\left(\sqrt{\sigma_{n} \sigma_{n+N}} \sin \theta\right) e^{\mathrm{i}\left(m+\frac{1}{2}\right)\left(\varphi+\zeta \frac{y}{\ell}\right)}, \\
a_{I, n+N}^{-}(v, \bar{v})= & \sum_{m \in \mathbb{Z}} x_{I}^{m, n} I_{\left|m-\frac{1}{2}\right|}\left(\sqrt{\sigma_{n} \sigma_{n+N}} \sin \theta\right) e^{\mathrm{i}\left(m-\frac{1}{2}\right)\left(\varphi+\zeta \frac{y}{\ell}\right)} .
\end{aligned}
$$

Using Eqs. (115) and (116) and the definition of $\mathcal{Z}_{I}$, we find that $\mathcal{Z}_{I}$ are now periodic in $\varphi$ and take the form

$$
\mathcal{Z}_{I}=e^{\frac{\mathrm{i} \alpha y}{2 \ell}} \sum_{m, n \in \mathbb{Z}} x_{I}^{m, n}(\ldots) e^{\mathrm{i} m\left(\varphi+\zeta \frac{y}{\ell}\right)+\frac{2 \pi i n y}{\beta \ell}}
$$


while previously they were anti-periodic in $\varphi$. Here, (..) is the combination of modified Bessel functions similar to that appearing in (124); it depends on which of the two cases, (151) and (152) or (153) and (154), we consider, and its precise form is not important. The four-dimensional action reduces to the same two-dimensional action (146) as before, but now with $\mathcal{Z}_{I}$ periodic in $\varphi$.

The one-loop determinant for hypermultiplets $Z_{\text {mat }}(u)$ might, of course, be modified by the defect. ${ }^{99}$ We are going to determine this indirectly now. Similar to the previous case, the path integral answer factorizes into an "index" part that counts operators and a "Casimir" part encoding the vacuum properties,

$$
Z_{\text {mat }}(u) \int \mathscr{D} \mathcal{Z}_{I} e^{-4 \pi \mathrm{i} \ell \int_{S_{\varphi}^{1} \times S_{y}^{1}} \mathrm{~d}^{2} w \varepsilon^{I I} Z_{I} \nabla_{\bar{w}} Z_{J}}=\int \mathscr{D} \mathscr{H} e^{-\mathscr{S}_{\text {mat }}[\mathscr{H}, u]}=Z_{\text {Cas }}(q, u) \mathscr{I}(q, u),
$$

where in our notation, we drop the dependence on $q=e^{-\beta(1+\mathrm{i} \zeta)}$ on the left.

The index $\mathscr{I}$ still counts Schur operators, simply because $\mathscr{Q}^{H}$ is conserved. However, the state-operator correspondence is slightly modified in the presence of a defect: the $E$ quantum number of a Schur state receives a $\pm \frac{1}{2}$ shift compared to the $E$ quantum number of the corresponding Schur operator (this is related to the shift of angular momentum in the presence of a defect, as mentioned in Ref. 23). One way to understand this is by going back to flat space. Specifically, we again replace $S^{3} \times S_{y}^{1}$ with $S^{3} \times \mathbb{R}$ and Weyl transform the latter cylinder to the $\mathbb{R}^{4}=\mathbb{C}_{z_{1}} \times \mathbb{C}_{z_{2}}$, with the chiral algebra plane being $\mathbb{C}_{z_{1}} \times\{0\}$ and the defect supported at $\{0\} \times \mathbb{C}_{z_{2}}$. Usually, to construct states in the radial quantization Hilbert space $\mathscr{H}_{S^{3}}$, we bring operators to the origin of $\mathbb{R}^{4}$ and observe what states they create in $\mathscr{H}_{S^{3}}$,

$$
\mathscr{O} \rightarrow|\mathscr{O}\rangle=\mathscr{O}(0)|0\rangle, \quad \text { where }|0\rangle \text { is the no - defect vacuum. }
$$

Because our defect is defined to be invisible for vector multiplets, this procedure is not modified for the Schur operators built out of gaugini. However, the free hypermultiplet Schur operators, ${ }^{100}$ which are $q_{11}, q_{12}$, and their $D_{++}$derivatives, do feel the presence of a defect. Specifically, for the two versions of the defect, they behave as (suppose we move them toward the origin along the $w=0$ plane)

$$
\begin{aligned}
& \text { Version I: } \quad q_{11}\left(z_{1}, \bar{z}_{1}\right)|0\rangle_{D} \sim \sqrt{r}\left|q_{11}\right\rangle_{D}+O\left(r^{3 / 2}\right) \\
& q_{12}\left(z_{1}, \bar{z}_{1}\right)|0\rangle_{D} \sim \frac{1}{\sqrt{r}}\left|q_{12}\right\rangle_{D}+O\left(r^{1 / 2}\right), \\
& \text { Version II: } \quad q_{11}\left(z_{1}, \bar{z}_{1}\right)|0\rangle_{D} \sim \frac{1}{\sqrt{r}}\left|q_{11}\right\rangle_{D}+O\left(r^{1 / 2}\right) \text {, } \\
& q_{12}\left(z_{1}, \bar{z}_{1}\right)|0\rangle_{D} \sim \sqrt{r}\left|q_{12}\right\rangle_{D}+O\left(r^{3 / 2}\right),
\end{aligned}
$$

where $|0\rangle_{D}$ is the defect vacuum and $r=\left|z_{1}\right|$ denotes the distance to the origin. Here, $\left|q_{11}\right\rangle_{D}$ and $\left|q_{12}\right\rangle_{D}$ denote the corresponding Schur states in the radial quantization Hilbert space with the defect. These are the states counted by the Schur index. The explicit factors of $r^{ \pm 1 / 2}$ show that the conformal dimensions (eigenvalues of $E$ ) of such states are shifted compared to those of the operators by $\pm \frac{1}{2}$. The R-charges are, of course, not modified, and so this shift is straightforwardly incorporated into the Schur index $\operatorname{Tr}(-1)^{F} q^{E-R} u^{f}$ of a free hypermultiplet coupled to the background flat connection. Specifically, every single-letter contribution should be additionally multiplied by $q^{1 / 2}$ or $q^{-1 / 2}$, reflecting the respective shift of $E$. As before, we consider the cases $N=0 \bmod 2$ and $N=1 \bmod 2$ and find the following:

$$
\begin{aligned}
\text { The case of } N=0 \bmod 2: & \\
\text { Version I: } & \mathscr{I}(q, u)=\prod_{w \in \mathbf{R}} \frac{1}{\left[\left(q u^{w} ; q\right)\left(u^{-w} ; q\right)\right]^{1 / 2}}, \\
\text { Version II: } & \mathscr{I}(q, u)=\prod_{w \in \mathbf{R}} \frac{1}{\left[\left(u^{w} ; q\right)\left(q u^{-w} ; q\right)\right]^{1 / 2}},
\end{aligned}
$$

The case of $N=1 \bmod 2$ :

$$
\begin{array}{cl}
\text { Version I: } & \mathscr{I}(q, u)=\prod_{w \in \mathbf{R}} \frac{1}{\left[\left(-q u^{w} ; q\right)\left(-u^{-w} ; q\right)\right]^{1 / 2}}, \\
\text { Version II: } & \mathscr{I}(q, u)=\prod_{w \in \mathbf{R}} \frac{1}{\left[\left(-u^{w} ; q\right)\left(-q u^{-w} ; q\right)\right]^{1 / 2}} .
\end{array}
$$

In fact, ${ }^{101} \sum_{w \in \mathbf{R}} w=0$, and one can easily check that in both cases, "Version I" and "Version II" answers coincide, and so there is no need to distinguish them in the following. We can also compute the two-dimensional path integral for symplectic bosons periodic in $\varphi$ and either periodic or anti-periodic in $y,{ }^{102}$ 
Periodic in $y$ :

$$
\begin{aligned}
& \int \mathscr{D} Z_{I} e^{-4 \pi \mathrm{i} \ell \int_{S_{y}^{1} \times S_{\varphi}^{1}} \mathrm{~d}^{2} w \varepsilon^{I J} Z_{I} \nabla_{\bar{w}} Z_{J}}=q^{-\frac{\operatorname{dim} \mathbf{R}}{24}} \prod_{w \in \mathbf{R}} \frac{\left[u^{w / 2}-u^{-w / 2}\right]^{-1 / 2}}{\left[\left(q u^{w} ; q\right)\left(q u^{-w} ; q\right)\right]^{1 / 2}}, \\
& \text { Anti - periodic in } y: \\
& \int \mathscr{D} Z_{I} e^{-4 \pi i \ell \int_{S_{y}^{1} \times S_{\varphi}^{1}} \mathrm{~d}^{2} w \varepsilon^{I I} Z_{I} \nabla_{\bar{w}} Z_{J}}=q^{-\frac{\operatorname{dim} \mathbf{R}}{24}} \prod_{w \in \mathbf{R}} \frac{\left[u^{w / 2}+u^{-w / 2}\right]^{-1 / 2}}{\left[\left(-q u^{w} ; q\right)\left(-q u^{-w} ; q\right)\right]^{1 / 2}} .
\end{aligned}
$$

We find that the former matches the defect Schur index for $N=0 \bmod 2$ if we include the appropriate four-dimensional Casimir energy factor, while the latter matches the $N=1 \bmod 2$ case of the defect Schur index under the same assumption. We claim that the four-dimensional Casimir factor takes the following form in all cases:

$$
Z_{\text {Cas }}(q, u)=q^{-\frac{\operatorname{dim} \mathbf{R}}{24}-\frac{1}{4} \sum_{w \in \mathrm{R}}\langle w, a\rangle^{2}}
$$

where the additional term $\frac{1}{4} \sum_{w \in \mathbf{R}}\langle w, a\rangle^{2}$ was included by hand to cancel similar terms in the vector multiplet Casimir energy (so that the total two-dimensional answer matches the four-dimensional answer precisely). In particular, this implies that the matter one-loop determinant takes the same form as in the absence of defects,

$$
Z_{\mathrm{mat}}(u)=q^{-\frac{1}{4} \sum_{w \in \mathbf{R}}\langle w, a\rangle^{2}}
$$

which heuristically makes sense because half of the hypermultiplet scalars receive a shift of $+\frac{1}{2}$ and half are shifted by $-\frac{1}{2}$, making it plausible for the total contribution to stay the same. It would be instructive to verify this by a direct evaluation of the one-loop determinant in the presence of a defect.

To summarize, we claim here that the precise equality of four-dimensional and two-dimensional path integrals (145) continues to hold in the presence of monodromy defects, with the only modification that the symplectic bosons become periodic in $\varphi$. Note, however, that generally both sides in (145) might be infinite: in the two-dimensional language, this is caused by the zero modes present in the Ramond sector of the $\beta-\gamma$ system. For this reason, we must turn on generic flavor fugacities, which render both sides in (145) finite. We expect the equality in (145) to remain true if we include the appropriate "flavor Casimir term" given in (148).

\section{Deformations}

Let us now turn toward a short survey of the possible "standard" deformations of our localization construction and whether they preserve the two-dimensional chiral algebra construction (i.e., whether or not $\mathscr{Q}^{H}$ is preserved). We have already argued that deformations away from the Schur fugacities (i.e., $\alpha, \zeta \neq 0$ ) are only allowed through the addition of $\zeta$ and $\alpha$ as in (48). In this section, we briefly explore the addition of other types of deformations including masses, Fayet-Iliopoulos (FI) parameters, and holonomies.

We are exclusively dealing with superconformal field theories, but one could ask whether our construction admits a non-conformal mass deformation. To answer this, note that the conservation of $\mathscr{Q}^{H}$ requires that the $U(1)_{r}$ R-symmetry is preserved. On the other hand, supersymmetric twisted masses are introduced by giving a vev to a scalar $\phi$ in the background vector multiplet. This scalar has a $U(1)_{r}$-charge $r=1$, and thus, its vev explicitly breaks the $U(1)_{r}$ symmetry. Therefore, mass deformations are not available.

The inclusion of FI terms is also not possible for two separate reasons, both related to the $U(1)_{r}$ symmetry. One trivial reason is that they can only be introduced for Abelian factors of the gauge group, and there are no interacting four-dimensional $\mathscr{N}=2$ superconformal field theories with Abelian factors in the gauge group [any such theory would break $U(1)_{r}$ and conformal invariance quantum-mechanically, as one can see from (46)]. However, even classically, the standard FI term explicitly breaks $U(1)_{r}$. Indeed, supersymmetry allows the addition of ${ }^{67}$

$$
\mathscr{L}_{\mathrm{FI}}=\zeta\left\{\omega^{A B} D_{A B}-M(\phi+\bar{\phi})\right\}
$$

to $\mathscr{N}=2$ theories in conformal supergravity (with $T_{\mu \nu}=0$ ), if there exists an $S U(2)_{R}$-triplet background field $\omega^{A B}$, which satisfies equations

$$
\omega^{A B} \xi_{B}=\frac{1}{2} \sigma^{\mu} \mathscr{D}_{\mu} \bar{\xi}^{A}, \quad \omega^{A B} \bar{\xi}_{B}=\frac{1}{2} \bar{\sigma}^{\mu} \mathscr{D}_{\mu} \xi^{A}
$$

We see that the term $M(\phi+\bar{\phi})$ in $\mathscr{L}_{\text {FI }}$ explicitly breaks $U(1)_{r}$.

There exists a non-standard FI term on $S^{3} \times S^{1}$, which preserves $U(1)_{r}$ classically. In fact, this term is a dimensional uplift of a $\mathscr{Q}^{H}$ preserving FI term in three-dimensional $\mathscr{N}=4$ theories. ${ }^{50}$ It is given by the following insertion in the path integral: 


$$
\exp \left[S_{\text {F.I. }}\right]=\exp \left[\frac{\mathrm{i} K}{2 \pi^{2} \ell^{2}} \int_{S^{3} \times S^{1}} \operatorname{vol}_{S^{3}} \mathrm{~d} y\left(D_{12}+\frac{2}{\ell} A_{y}\right)\right]
$$

where $D_{12}$ and $A_{y}$ are fields in the Abelian vector multiplet. One can check that classically, such an insertion preserves all supersymmetries. Note that large gauge transformations require that the FI parameter is integer, $K \in \mathbb{Z}$. Such FI terms were previously considered in Refs. 60, 87 , and 103 , and we mention them for completeness. Of course, we already know that quantum-mechanically, there are no interacting theories with Abelian gauge factors that preserve $U(1)_{r}$, and so this type of FI term is not available either.

The class of deformations that $d o$ preserve all the symmetries required by our construction are flavor holonomies, briefly mentioned in Subsection III C. They are given by constant Abelian vevs for $A_{y}$ in the background vector multiplets, gauging flavor symmetries of the system. They correspond to introducing flavor fugacities in the Schur index, and the possibility of such a refinement was, of course, already mentioned in Ref. 9. Note also that such a deformation is a dimensional uplift of the $\mathscr{Q}^{H}$-invariant mass in three-dimensional $\mathscr{N}=4$ theories. ${ }^{50}$ The only effect flavor holonomies have on the two-dimensional action (146) is that they modify the covariant derivative acting on the symplectic bosons in an obvious way, i.e.,

$$
\nabla_{\bar{w}} \rightarrow \nabla_{\bar{w}}-\mathrm{i} \frac{a_{f}}{2 \beta}
$$

where $u_{f}=e^{\mathrm{i} a_{f}}$ is the flavor holonomy around $S_{y}^{1}$. When flavor holonomies are required to render the Schur index finite, we will allow $a_{f}$ to be complex (i.e., include both the holonomy and the "chemical potential"). We will give another interpretation of this deformation from the VOA point of view further below.

\section{THE TWO-DIMENSIONAL THEORY}

The main result of Sec. III C is the two-dimensional theory (146), which we derived through a localization computation with respect to the supercharge $\mathscr{Q}^{H}$ on $S^{3} \times S_{y}^{1}$. In this section, we are going to elucidate its various properties. We start by describing its integration cycle, then describe its propagators of the elementary fields, and finally discuss the effect of adding flavor holonomies.

\section{A. The integration cycle}

Note that the reality conditions (45) of the four-dimensional theory induce the reality properties of the symplectic bosons, i.e., they determine the integration cycle for the theory (146). We now briefly discuss this cycle.

First, we consider the case without the monodromy defect, that is, when $Z_{I}$ is anti-periodic in $\varphi$. Using the decomposition (122) of general solutions to the BPS equations and the reality conditions of the hypermultiplets (45), we can find the reality conditions for the modes $x_{I}^{m, n}$ in (122). For $N=0$, they simplify and take the following form:

$$
\left(x_{I}^{m, n}\right)^{*}=(-1)^{m} \varepsilon^{I J} x_{J}^{-m+1,-n} .
$$

For convenience, let us slightly redefine the modes in (122) so that the expression for $\mathcal{Z}_{I}$ reads

$$
\mathcal{Z}_{I}(\varphi, y)=e^{-\frac{\mathrm{i}(\zeta-\alpha) y}{2 \ell}-\frac{\mathrm{i}}{2} \varphi} \sum_{n, m \in \mathbb{Z}} c_{I}^{m, n} e^{\mathrm{i} m\left(\varphi+\zeta \frac{y}{\ell}\right)+\frac{2 \pi i n y}{\beta \ell}}
$$

Then, for $N=0$, the corresponding reality conditions for the $c_{I}^{m, n}$ modes become

$$
\left(c_{I}^{n, m}\right)^{*}=\mathrm{i} \varepsilon^{I J} c_{J}^{-m+1,-n} \frac{I_{m-1}\left(\sigma_{n}\right)-\mathrm{i} I_{m}\left(\sigma_{n}\right)}{I_{m-1}\left(\sigma_{n}\right)+\mathrm{i} I_{m}\left(\sigma_{n}\right)} .
$$

Note that $\frac{I_{m-1}\left(\sigma_{n}\right)-\mathrm{i} I_{m}\left(\sigma_{n}\right)}{I_{m-1}\left(\sigma_{n}\right)+\mathrm{i} I_{m}\left(\sigma_{n}\right)}$ is a phase that is closer to 1 for $m \geq 1$ and closer to -1 for $m \leq 0$-in other words, it is close to sgn $\left(m-\frac{1}{2}\right)$ (especially for large $|m|)$. Because the above equation defines a middle-dimensional integration cycle in the space of complexified fields, one expects the path integral to be invariant under small deformations of the cycle, as long as the convergence is preserved along the deformations. In particular, we expect that the above cycle can be deformed to a simpler one given by

$$
\left(c_{I}^{n, m}\right)^{*}=\mathrm{i} \operatorname{sgn}\left(m-\frac{1}{2}\right) \mathcal{E}^{I J} c_{J}^{-m+1,-n}
$$

We will now verify that this is indeed true and further find that for general $N \in \mathbb{Z}$, the analogous simplified integration cycle still has a concise expression given by

$$
\left(c_{I}^{m, n}\right)^{*}=\mathrm{i} \operatorname{sgn}\left(m-\frac{1}{2}\right) \varepsilon^{I J} c_{J}^{-m+1,-n-N} .
$$


To check this, we evaluate the two-dimensional symplectic boson action in terms of the modes $c_{I}^{m, n}$ in (175),

$$
\begin{aligned}
4 \pi \mathrm{i} \int & \mathrm{d} y \mathrm{~d} \varphi \varepsilon^{I J} Z_{I} \nabla_{\bar{w}} Z_{J}= \\
= & 4 \pi^{2} \mathrm{i} \beta \ell \sum_{m, n \in \mathbb{Z}} \varepsilon^{I J} c_{J}^{-m+1,-n-N}\left(m-\frac{1}{2}-\frac{2 \pi \mathrm{i}(n+N / 2)}{\beta}+\mathrm{i} \frac{a}{\beta}\right) c_{I}^{m, n} \\
= & 4 \pi^{2} \beta \ell \sum_{m, n \in \mathbb{Z}}\left(c_{I}^{m, n}\right)^{*} \operatorname{sgn}\left(m-\frac{1}{2}\right)\left(m-\frac{1}{2}-\frac{2 \pi \mathrm{i}(n+N / 2)}{\beta}+\mathrm{i} \frac{a}{\beta}\right) c_{I}^{m, n},
\end{aligned}
$$

where going from the second to the third line we used (178). Thus, we see that the real part of this action is positive definite along the integration cycle (178), ensuring convergence of the path integral, while the imaginary part is sign indefinite. Using standard Morse theory arguments $^{104,105}$ (see Ref. 50 for an application close to the current context), one can check that the integration cycle (178) (up to convergencepreserving deformations) is the only allowed one in this theory, as long as we keep a purely real. ${ }^{106}$ We do not consider alternative integration cycles for $a$ until Sec. V, where they are expected to be relevant for the study of modularity and general surface defects in four dimensions (which reduce to the modules of the two-dimensional chiral algebra).

As we explained previously, in the presence of the monodromy creating defect, the symplectic bosons become periodic in $\varphi$. The Fourier expansion thus takes the form

$$
Z_{I}(\varphi, y)=e^{\frac{\mathrm{i} \alpha y}{2 \ell}} \sum_{n, m \in \mathbb{Z}} s_{I}^{m, n} e^{\mathrm{i} i\left(\varphi+\zeta \frac{y}{\ell}\right)+\frac{2 \pi i n y}{\beta \ell}},
$$

and we can evaluate the two-dimensional action in terms of modes $s_{I}^{m, n}$ as

$$
\begin{aligned}
& 4 \pi \mathrm{i} \int \mathrm{d} y \mathrm{~d} \varphi \varepsilon^{I J} Z_{I} \nabla_{\bar{w}} Z_{J} \\
& =4 \pi^{2} \mathrm{i} \beta \ell \sum_{m, n} \varepsilon^{I J} s_{J}^{-m,-n-N}\left(m-\frac{2 \pi \mathrm{i}}{\beta}\left(n+\frac{N}{2}\right)+\mathrm{i} \frac{a}{\beta}\right) s_{I}^{m, n} .
\end{aligned}
$$

We see that the integration cycle should be

$$
\left(s_{I}^{m, n}\right)^{*}=\mathrm{i} \operatorname{sgn}(m) \varepsilon^{I J} s_{J}^{-m,-n-N},
$$

where we choose to extend $\operatorname{sgn}(0)=+1$. The action then becomes

$$
4 \pi^{2} \beta \ell \sum_{m, n}\left(s_{I}^{m, n}\right)^{*} \operatorname{sgn}(m)\left(m-\frac{2 \pi \mathrm{i}}{\beta}\left(n+\frac{N}{2}\right)+\mathrm{i} \frac{a}{\beta}\right) s_{I}^{m, n} .
$$

Recall that the full theory (145) involves integration over the holonomy variable $a$, and the presence of the zero mode $m=0$ in (183) can cause this integration to diverge, which is typical for characters in the Ramond sector. As we have mentioned previously, this divergence is cured by turning on flavor holonomies, $a \rightarrow a+a_{f}$, and furthermore, we should keep them complex. Equation (183) manifests that positive definiteness of the action requires $\operatorname{Im}\left(a_{f}\right)$ to be small enough to not alter the convergence for $m \neq 0$ modes. Importantly, for convergence of the integral over $m=0$ modes, with the choice $\operatorname{sgn}(0)=+1$, we should keep the imaginary part of $a_{f}$ negative,

$$
\operatorname{Im}\left(a_{f}\right)<0 .
$$

\section{B. Green's functions}

In this subsection, we compute the torus propagators of the elementary two-dimensional fields. For symplectic bosons, we do it in all four spin structures described above. The equations in this subsection are reminiscent of those appearing in standard treatments of genus-one superstring amplitudes. Nevertheless, we provide a detailed derivation here, both for completeness and because the ghost systems we deal with are slightly different from the ones appearing in superstrings and sometimes require an extra amount of care.

\section{Fermionic propagator}

We start by expanding the small $b c$ ghosts in Fourier modes,

$$
b=\sum_{\substack{m, n \in \mathbb{Z} \\ m \neq 0}} b_{m, n} e^{\mathrm{i} m\left(\varphi+\zeta \frac{y}{\ell}\right)+\frac{2 \pi i n y}{\beta \ell}}, \quad c=\sum_{\substack{m, n \in \mathbb{Z} \\ m \neq 0}} c_{m, n} e^{\mathrm{i} m\left(\varphi+\zeta \frac{y}{\ell}\right)+\frac{2 \pi i n y}{\beta \ell}},
$$


where the $m=0$ modes are absent due to the delta-functions in (88). The action then takes the form

$$
\mathscr{S}_{2 \mathrm{~d}}^{(b c)}=-\pi \beta \ell \operatorname{Tr} \sum_{\substack{m, n \in \mathbb{Z} \\ m \neq 0}} b_{-m,-n}\left(m-\frac{2 \pi \mathrm{i} n}{\beta}+\mathrm{i} \frac{a}{\beta}\right) c_{m, n}
$$

and the propagator is given by

$$
\begin{aligned}
\left\langle c^{A}\left(w_{1}\right) b^{B}\left(w_{2}\right)\right\rangle & =P^{A B}\left(w_{1}-w_{2}, \bar{w}_{1}-\bar{w}_{2}\right), \\
P(w, \bar{w}) & =-\frac{1}{\pi \beta \ell} \sum_{\substack{m, n \in \mathbb{Z} \\
m \neq 0}} \frac{e^{\mathrm{i} m\left(\varphi+\zeta \frac{y}{\ell}\right)+\frac{2 \pi i n y}{\beta \ell}}}{m-\frac{2 \pi \mathrm{in} n}{\beta}+\mathrm{i} \frac{a}{\beta}} \equiv \mathscr{G}(w, \bar{w})+g(w, \bar{w}),
\end{aligned}
$$

with

$$
\begin{aligned}
& \mathscr{G}(w, \bar{w})=-\frac{1}{\pi \beta \ell} \sum_{m, n \in \mathbb{Z}} \frac{e^{\mathrm{i} m\left(\varphi+\zeta \frac{y}{\ell}\right)+\frac{2 \pi i n y}{\beta \ell}}}{m-\frac{2 \pi \mathrm{i} n}{\beta}+\mathrm{i} \frac{a}{\beta}}, \\
& g(w, \bar{w})=-\frac{1}{\pi \beta \ell} \sum_{n \in \mathbb{Z}} \frac{e^{\frac{2 \pi i n y}{\beta \ell}}}{\frac{2 \pi i n}{\beta}-\mathrm{i} \frac{a}{\beta}},
\end{aligned}
$$

and where $A, B$ are the adjoint indices, and $P$ is a $|G| \times|G|$ matrix determined by the diagonal matrix $a \in \mathfrak{g}$. We have introduced an auxiliary Green's function $\mathscr{G}$ that includes zero modes (and thus describes the full $b c$ ghost system) and the correction term $g(w, \bar{w})$ that removes $m=0$ modes. This correction term is essentially a propagator of the one-dimensional analog of the $b c$ system.

The function $\mathscr{G}(w, \bar{w})$ satisfies an equation

$$
\nabla_{\bar{w}} \mathscr{G}(w, \bar{w})=\delta(\varphi) \delta(y)
$$

and is required to be doubly periodic on the torus, namely, it obeys [cf. (67)]

$$
\mathscr{G}(w+2 \pi \mathrm{i} \tau, \bar{w}-2 \pi \mathrm{i} \bar{\tau})=\mathscr{G}(w+2 \pi \mathrm{i}, \bar{w}-2 \pi \mathrm{i})=\mathscr{G}(w, \bar{w}),
$$

where

$$
\tau=\frac{\mathrm{i} \beta}{2 \pi}(1+\mathrm{i} \zeta)
$$

We can also define the function $\mathscr{G}_{0}(w, \bar{w})$,

$$
\mathscr{G}(w, \bar{w})=e^{\mathrm{i} \frac{a}{2 \beta}(w+\bar{w})} \mathscr{G}_{0}(w, \bar{w}),
$$

which satisfies a simpler equation but has a twisted periodicity along $\operatorname{Re}(w)$,

$$
\begin{aligned}
\partial_{\bar{w}} \mathscr{G}_{0}(w, \bar{w}) & =\delta(\varphi) \delta(y), \\
\mathscr{G}_{0}(w+2 \pi \mathrm{i} \tau, \bar{w}-2 \pi \mathrm{i} \bar{\tau}) & =e^{\mathrm{i} a} \mathscr{G}_{0}(w, \bar{w}) .
\end{aligned}
$$

The equation implies that the function is holomorphic away from $w=0$ and has a simple pole at $w=0$ with residue $(\pi \ell)^{-1}$. This would be impossible for $a=0$, since the function would have to be elliptic, and such functions have poles of total order at least two. However, it is possible at generic $a$. The standard way to construct functions of a given periodicity on elliptic curves is by taking ratios of Jacobi theta-functions. The basic Jacobi theta-function is defined as follows:

$$
\vartheta(z ; \tau)=\sum_{n \in \mathbb{Z}} \exp \left(\mathrm{i} \pi n^{2} \tau+2 \pi \mathrm{i} n z\right) .
$$

In the present case, the relevant function is

$$
\theta_{1}(z ; \tau)=-\exp \left(\frac{1}{4} \mathrm{i} \pi \tau+\mathrm{i} \pi(z+1 / 2)\right) \vartheta\left(z+\frac{1}{2} \tau+\frac{1}{2} ; \tau\right) .
$$


It has a simple zero at $z=0$ and satisfies

$$
\theta_{1}(z+n+m \tau ; \tau)=e^{\mathrm{i} \pi(n-m)-\mathrm{i} \pi m^{2} \tau-2 \mathrm{i} \pi m z} \theta_{1}(z ; \tau) .
$$

One can easily check that the function

$$
\frac{\theta_{1}\left(\frac{w}{2 \pi \mathrm{i}}-\frac{a}{2 \pi} ; \tau\right)}{\theta_{1}\left(\frac{w}{2 \pi \mathrm{i}} ; \tau\right)}
$$

has a simple pole at $w=0$ and satisfies the periodicity as required for $\mathscr{G}_{0}$. If we further normalize it to have a residue $(\pi \ell)^{-1}$ at $w=0$, as required by (195), we find

$$
\mathscr{G}_{0}(w)=\frac{1}{2 \pi^{2} \mathrm{i} \ell} \cdot \frac{\theta_{1}^{\prime}(0 ; \tau)}{\theta_{1}\left(-\frac{a}{2 \pi} ; \tau\right)} \frac{\theta_{1}\left(\frac{w}{2 \pi \mathrm{i}}-\frac{a}{2 \pi} ; \tau\right)}{\theta_{1}\left(\frac{w}{2 \pi \mathrm{i}} ; \tau\right)},
$$

where $\theta_{1}^{\prime}$ denotes the derivative in the first argument of $\theta_{1}$. This is actually a unique solution for $\mathscr{G}_{0}$. Any other solution, according to (195), would differ from (201) by a holomorphic function $f(w)$ satisfying $f(w+2 \pi \mathrm{i})=f(w)$ and $f(w+2 \pi \mathrm{i} \tau)=e^{\mathrm{i} a} f(w)$. Such a function extends to a bounded holomorphic function on $\mathbb{C}$, which thus must be a constant. Additionally, $f(w+2 \pi \mathrm{i} \tau)=e^{\mathrm{i} a} f(w)$ (together with $a$ being generic) implies that it ought to vanish.

We next turn to the correction term $g(w, \bar{w})$. Summing its Fourier series, we find

$$
g(w, \bar{w})=\frac{1}{\pi \ell} \cdot \frac{e^{\mathrm{i} a \frac{y}{\beta \ell}}}{e^{\mathrm{i} a}-1} \quad \text { for } \quad 0<y<\beta \ell .
$$

Note that this function is discontinuous on the whole circle $S_{y}^{1}$, namely, it has a jump at $y=0 \bmod \beta \ell \mathbb{Z}$. However, as explained in the paragraph after Eqs. (91), we must keep operator insertions separated in the $y$ direction, i.e., work in the interval $0<y<\beta \ell$, where $g(w, \bar{w})$ is smooth. Collecting all the pieces, we find the propagator

$$
P(w, \bar{w})=\frac{1}{2 \pi^{2} \mathrm{i} \ell} e^{\mathrm{i} \frac{a}{2 \beta}(w+\bar{w})} \frac{\theta_{1}^{\prime}(0 ; \tau)}{\theta_{1}\left(-\frac{a}{2 \pi} ; \tau\right)} \frac{\theta_{1}\left(\frac{w}{2 \pi \mathrm{i}}-\frac{a}{2 \pi} ; \tau\right)}{\theta_{1}\left(\frac{w}{2 \pi \mathrm{i}} ; \tau\right)}+\frac{1}{\pi \ell} \frac{e^{\mathrm{i} \frac{a}{2 \beta}(w+\bar{w})}}{e^{\mathrm{i} a}-1}
$$

for $0<\operatorname{Re}(w)<\beta$. In the small $b c$ ghost system, all vertex operators are constructed from $b, \nabla_{w} c$, and their derivatives, so it is more useful to know $\left\langle\nabla_{w} c(w, \bar{w}) b(0)\right\rangle=\nabla_{w} P(w, \bar{w})$. We note that $\nabla_{w} e^{\mathrm{i} \frac{a}{2 \beta}(w+\bar{w})}=0$, which implies that

$$
\left\langle\nabla_{w} c(w, \bar{w}) b(0)\right\rangle=\frac{1}{2 \pi^{2} \mathrm{i} \ell} \cdot e^{\mathrm{i} \frac{a}{2 \beta}(w+\bar{w})} \frac{\theta_{1}^{\prime}(0 ; \tau)}{\theta_{1}\left(-\frac{a}{2 \pi} ; \tau\right)} \partial_{w} \frac{\theta_{1}\left(\frac{w}{2 \pi \mathrm{i}}-\frac{a}{2 \pi} ; \tau\right)}{\theta_{1}\left(\frac{w}{2 \pi \mathrm{i}} ; \tau\right)} .
$$

In fact, this is the expression that must be compared against the four-dimensional correlator of twisted-translated operators $\langle\lambda(w, \bar{w}) \widetilde{\lambda}(0)\rangle$ computed in Ref. 42. To do this, we define a new variable $z=-\mathrm{i} w$ and note that

$$
\theta_{1}^{\prime}(0, \tau)=2 \pi \eta(\tau)^{3} .
$$

Then, we obtain

$$
\left\langle\nabla_{z} c(z, \bar{z}) b(0)\right\rangle=\frac{1}{\pi \mathrm{i} \ell} \eta(\tau)^{3} \cdot e^{-\mathrm{i} \frac{a}{2 \pi} \frac{z-\bar{z}}{\tau-\bar{\tau}}} \partial_{z} \frac{\theta_{1}\left(-\frac{a}{2 \pi}+\frac{z}{2 \pi} ; \tau\right)}{\theta_{1}\left(\frac{z}{2 \pi} ; \tau\right) \theta_{1}\left(-\frac{a}{2 \pi} ; \tau\right)} .
$$

Upon rescaling fields by numerical factors, we see complete agreement with (4.24) of Ref. 42, which implies that the $b c$ ghost action in (88) is the correct one, namely, it fully reproduces all the necessary correlation functions in the VOA. Finally, we should also note that on components of the $b c$ fields valued in the Cartan subalgebra, $a$ acts by zero, and one can check that the above formula still applies as it has a well-defined $a \rightarrow 0$ limit.

\section{Bosonic propagators}

We now repeat this procedure for the symplectic bosons. In the absence of monodromy defects, the Fourier expansion of the bosonic two-dimensional action is given in (179), while the presence of a defect modifies it to (181). Throughout this section, $a \in \mathfrak{g}$ is assumed to be a diagonal matrix acting in the matter representation $\mathbf{R}$. To avoid clutter, we still write it as $a$, with the understanding that on a weight $\rho \in \mathbf{R}$, it acts as a multiplication by $\rho(a)$. The two-point function in all cases is

$$
\left\langle Z_{I}\left(w_{1}\right) Z_{J}\left(w_{2}\right)\right\rangle=\frac{\mathrm{i}}{4 \pi \ell} \varepsilon_{I J} B\left(w_{1}-w_{2}, \bar{w}_{1}-\bar{w}_{2}\right),
$$


where the function $B(w, \bar{w})$ has a double Fourier series representation,

$$
\begin{aligned}
\text { Without defect: } \quad B(w, \bar{w}) & =\frac{1}{\pi \beta} \sum_{m, n \in \mathbb{Z}} \frac{e^{\mathrm{i}\left(m-\frac{1}{2}\right)\left(\varphi+\zeta \frac{y}{\ell}\right)+2 \pi \mathrm{i}\left(n+\frac{N}{2}\right) \frac{y}{\beta \ell}}}{m-\frac{1}{2}-\frac{2 \pi \mathrm{i}}{\beta}\left(n+\frac{N}{2}\right)+\mathrm{i} \frac{a}{\beta}}, \\
\text { With defect: } \quad B(w, \bar{w}) & =\frac{1}{\pi \beta} \sum_{m, n \in \mathbb{Z}} \frac{e^{\mathrm{i} m\left(\varphi+\zeta \frac{y}{\ell}\right)+2 \pi \mathrm{i}\left(n+\frac{N}{2}\right) \frac{y}{\beta \ell}}}{m-\frac{2 \pi \mathrm{i}}{\beta}\left(n+\frac{N}{2}\right)+\mathrm{i} \frac{a}{\beta}} .
\end{aligned}
$$

Of course, the function $B(w, \bar{w})$ is the Green's function of $\nabla_{\bar{w}}$, i.e.,

$$
\begin{aligned}
\text { Without defect: } & \nabla_{\bar{w}} B(w, \bar{w})=-e^{-\frac{i}{2} \varphi+\mathrm{i} \pi(N-M) \frac{y}{\beta \ell}} \ell \delta(\varphi) \delta(y), \\
\text { With defect: } & \nabla_{\bar{w}} B(w, \bar{w})=-e^{\mathrm{i} \pi N \frac{y}{\beta \ell}} \ell \delta(\varphi) \delta(y) .
\end{aligned}
$$

The delta functions on the right-hand side are periodic, and the exponentials in front of them are necessary to match the periodicity of the left-hand side. The periodicity of $B$ in $\varphi$ is determined by the presence of a defect, while its periodicity in $y$ is determined by $N$ and $M$.

As before, the form of the covariant derivative $\nabla_{\bar{w}}=\partial_{\bar{w}}-\frac{i}{2 \beta}$ suggests defining $B_{0}$,

$$
B(w, \bar{w})=e^{i \frac{a}{2 \beta}(\bar{w}+w)} B_{0}(w) .
$$

Equations (210) and (211) then reduce to the following:

$$
\begin{aligned}
\text { Without defect: } & \partial_{\bar{w}} B_{0}(w)=-e^{-\frac{i}{2} \varphi+\mathrm{i} \pi(N-M) \frac{y}{\beta \ell}} \ell \delta(\varphi) \delta(y), \\
\text { With defect: } & \partial_{\bar{w}} B_{0}(w)=-e^{\mathrm{i} \pi N \frac{y}{\beta \ell}} \ell \delta(\varphi) \delta(y),
\end{aligned}
$$

implying that $B_{0}$ is always meromorphic with a simple pole at $w=0$ with residue $\left(-\pi^{-1}\right)$. It satisfies twisted periodicity along $y$ due to the explicit factor of $e^{\frac{i}{\beta e}(w+\bar{w})}$ and acquires an additional sign, either $(-1)^{N-M}$ or $(-1)^{N}$, upon going around $S_{y}^{1}$.

To summarize, the periodicity properties of $B_{0}$ are given as follows:

$$
\begin{array}{rll}
\text { Without defect: } & B_{0}(w+2 \pi \mathrm{i})=-B_{0}(w), & B_{0}(w+2 \pi \mathrm{i} \tau)=(-1)^{N-M} e^{\mathrm{i} a} B_{0}(w), \\
\text { With defect: } & B_{0}(w+2 \pi \mathrm{i})=B_{0}(w), & B_{0}(w+2 \pi \mathrm{i} \tau)=(-1)^{N} e^{\mathrm{i} a} B_{0}(w),
\end{array}
$$

where

$$
\tau=\frac{\mathrm{i} \beta}{2 \pi}(1+\mathrm{i} \zeta) .
$$

Again, we can easily write a ratio of theta-functions satisfying these properties and having a simple pole at $w=0$ with residue $\left(-\pi^{-1}\right)$. To this end, we recall the notation $\left(v_{1}, v_{2}\right)$ for the spin structure on $S_{\varphi}^{1} \times S_{y}^{1}$; symplectic bosons pick up a phase $e^{\mathrm{i} \pi v_{1}}$ upon going around $S_{\varphi}^{1}$ and a phase $e^{\mathrm{i} \pi v_{2}}$ around $S_{y}^{1}$. Therefore, we have ${ }^{107}$ the following:

$$
\begin{array}{rll}
\text { Without defect: } & v_{1}=1, & v_{2}=N-M \bmod 2, \\
\text { With defect: } & v_{1}=0, & v_{2}=N \bmod 2 .
\end{array}
$$

Using these notations, the answer is

$$
B_{0}(w)=\frac{\mathrm{i}}{2 \pi^{2}} \frac{\theta_{1}^{\prime}(0 ; \tau)}{\vartheta_{1-v_{1}, 1-v_{2}}\left(-\frac{a}{2 \pi} ; \tau\right)} \times \frac{\vartheta_{1-v_{1}, 1-v_{2}}\left(\frac{w}{2 \pi \mathrm{i}}-\frac{a}{2 \pi} ; \tau\right)}{\theta_{1}\left(\frac{w}{2 \pi i} ; \tau\right)},
$$

written in terms of the standard half-period theta functions,

$$
\vartheta_{n, m}(z ; \tau)=\exp \left(\frac{n}{4} \mathrm{i} \pi \tau+n \mathrm{i} \pi\left(z+\frac{m}{2}\right)\right) \vartheta\left(z+\frac{n}{2} \tau+\frac{m}{2} ; \tau\right) .
$$

Utilizing the basic property $9(z+n+m \tau ; \tau)=\exp \left(-\mathrm{i} \pi m^{2} \tau-2 \pi \mathrm{i} m z\right) 9(z ; \tau)$, one can check that $(220)$ satisfies the correct periodicity in all four cases (215) and (216). We prove the uniqueness of solution (220) as before. Because it has the correct residue at the (only) pole $w=0$, any other 
solution for $B_{0}$ would differ by a holomorphic function $f(w)$, obeying $f(w+2 \pi \mathrm{i} n+2 \pi \mathrm{i} \tau m)=(-1)^{n v_{1}+m v_{2}} e^{\mathrm{i} a} f(w)$. Such a function extends to a bounded holomorphic function on $\mathbb{C}$ and so is constant. Again, for generic $a$, this constant can only be zero.

Thus, we obtain the propagator of symplectic bosons for the arbitrary spin structure $\left(v_{1}, v_{2}\right)$,

$$
\begin{aligned}
B^{\left(v_{1}, v_{2}\right)}(w, \bar{w}) & =e^{\mathrm{i} \frac{a}{2 \beta}(\bar{w}+w)} \frac{\mathrm{i}}{2 \pi^{2}} \cdot \frac{\theta_{1}^{\prime}(0 ; \tau)}{\vartheta_{1-v_{1}, 1-v_{2}}\left(-\frac{a}{2 \pi} ; \tau\right)} \cdot \frac{\vartheta_{1-v_{1}, 1-v_{2}}\left(\frac{w}{2 \pi \mathrm{i}}-\frac{a}{2 \pi} ; \tau\right)}{\theta_{1}\left(\frac{w}{2 \pi \mathrm{i}} ; \tau\right)} \\
& =e^{\frac{a}{2 \pi} \overline{\bar{\tau}+w}} \frac{\mathrm{i}}{\pi} \eta(\tau)^{3} \cdot \frac{\vartheta_{1-v_{1}, 1-v_{2}}\left(\frac{w}{2 \pi \mathrm{i}}-\frac{a}{2 \pi} ; \tau\right)}{\vartheta_{1-v_{1}, 1-v_{2}}\left(-\frac{a}{2 \pi} ; \tau\right) \theta_{1}\left(\frac{w}{2 \pi \mathrm{i}} ; \tau\right)} .
\end{aligned}
$$

It is worth noting that $\left(v_{1}, v_{2}\right)=(1,0)$ is the spin structure considered in Ref. 42, and Eq. (222) agrees with their answer in this case.

\section{Flavor deformations and screenings}

The expressions for the two-point functions derived in Subsection IV B can also serve as the two-point functions when we switch on background holonomies for flavor symmetries. This corresponds to treating some components in $a$ as vevs for flavor symmetries, i.e., we now replace $a$ by $a+a_{f} \in \mathfrak{g} \oplus \mathfrak{f}$, where $\mathfrak{f}$ is the flavor symmetry Lie algebra. Both $a$ and $a_{f}$ are valued in the Cartan.

Operators that are charged under the Cartan $\mathfrak{t}_{f}$ of $\mathfrak{f}$ acquire non-holomorphic dependence when $a_{f} \neq 0$. Indeed, as follows from (222), a correlator of two such operators, e.g., $\left\langle\mathscr{O}_{\rho}(w, \bar{w}) \mathscr{O}_{-\rho}(0,0)\right\rangle$, is proportional to $e^{\frac{\rho\left(a_{f}\right)}{2 \pi} \frac{\bar{w}+w}{\bar{\tau}-\tau}}$, where $\rho \in \mathfrak{t}_{f}^{*}$ is a flavor weight. ${ }^{108}$ We might still choose to focus on the holomorphic sector of a theory with generic holonomies $a_{f} \neq 0$ turned on; it is formed by $\mathfrak{t}_{f}$-invariant Schur operators and provides a consistent truncation $V^{\mathfrak{t}_{f}}$ of the full VOA $V$.

In fact, such a truncation is familiar in the study of vertex operator algebras. Before describing it, first recall that when a four-dimensional superconformal field theory has flavor symmetry $\mathfrak{f}$ with four-dimensional central charge $k_{4 \mathrm{~d}}$, the corresponding VOA $V$ contains an affine Kac-Moody subalgebra,

$$
\widehat{\mathfrak{f}}_{k_{2 \mathrm{~d}}} \subset V,
$$

whose two-dimensional level is $k_{2 \mathrm{~d}}=-\frac{k_{4 \mathrm{~d}}}{2} .{ }^{9}$ This $\widehat{\mathfrak{f}}_{k_{2 \mathrm{~d}}}$ is generated by currents corresponding to the four-dimensional moment map operators. Suppose the currents $J_{i}(w), i=1 \ldots \mathrm{rk} \mathfrak{f}$, correspond to the Cartan elements of $\mathfrak{f}$. We define their respective charges,

$$
S_{i}=\oint \mathrm{d} w J_{i}(w)
$$

and the $\mathfrak{t}_{f}$-invariant subsector of the VOA $V$ can be determined as their common kernel,

$$
V^{\mathfrak{t}_{f}}=\bigcap_{i=1}^{\mathrm{rkf} f} \operatorname{ker} S_{i},
$$

i.e., $V^{\mathfrak{t}_{f}}$ is formed by the vertex operators commuting with all $S_{i}$ 's. Note that $V^{\mathfrak{t}_{f}}$ obviously contains the Virasoro element of $V$. This is an example of screening; such $S_{i}$ are known as screening charges, and $J_{i}(w)$ are called screening currents (see Ref. 109 for a general construction).

Therefore, we conclude that turning on flavor holonomies corresponds, at the level of the chiral algebra, to screening by the Cartan elements of the flavor symmetry. Screening is one of the standard operations for extracting vertex subalgebras. Another standard operationthe BRST reduction (which should be viewed as the VOA analog of quotient) - was previously identified as the two-dimensional avatar of four-dimensional gauging. ${ }^{9}$ While BRST reduction involves severe constraints on the matter content reflecting superconformal invariance in four dimensions and nilpotency of the BRST charge in two dimensions, no such constraints are necessary for screenings. Therefore, by turning on flavor holonomies, one, at least formally, significantly enlarges the class of VOAs appearing in four-dimensional superconformal field theories. Of course, this enlargement is somewhat formal because we simply consider various sub-VOAs of the larger VOA that exists when all flavor holonomies are zero. Nevertheless, this observation might be important for understanding various properties (in particular, modularity) of the flavored Schur index.

Note also that although the non-holomorphic factors cancel in $V^{t_{f}}$, the holonomies $a_{f}$ still appear in the arguments of various thetafunctions in (222). Thus, the corresponding torus correlation functions may still depend on $a_{f}$. In other words, in general, the $a_{f}$ parameterize a family of torus correlation functions for $V^{\mathfrak{t}_{f}}$.

\section{Example of a free hypermultiplet}

To illustrate the above ideas, let us consider the simplest example of a single free four-dimensional hypermultiplet. We turn on generic holonomy $a_{f} \in \mathbb{R} / 2 \pi \mathbb{Z}$ for its $U(1)_{F} \subset S U(2)_{F}$ flavor symmetry. The corresponding VOA is, as is well-known, a single symplectic boson (or a weight $-\frac{1}{2} \beta-\gamma$ system), and $U(1)_{F}$ corresponds to its ghost number symmetry. Specifically, the full VOA $V$ (placed on $\mathbb{C}$ ) is generated by

$$
\beta(z), \quad \gamma(z) \quad \text { with } \quad \beta(z) \gamma(0) \sim \frac{1}{z}
$$


and the screening is defined by the ghost number current,

$$
J(z)=: \beta(z) \gamma(z):
$$

The sub-VOA $V^{\mathfrak{u}(1)_{F}}$ annihilated by $S=\oint \mathrm{d} w: \beta(w) \gamma(w)$ : is formed by words built from $\beta(w), \gamma(w)$ and their derivatives such that each word has an equal number of $\beta$ 's and $\gamma$ 's, e.g., $J$ itself. It is quite easy to give a simpler description for this sub-VOA in the present case using the bosonization isomorphism. Recall that for the $\beta-\gamma$ system, it is given by

$$
\beta \cong e^{-\phi+\chi} \partial \chi, \quad \gamma \cong e^{\phi-\chi},
$$

where $\chi$ and $\phi$ are chiral bosons satisfying

$$
\phi(z) \phi(0) \sim-\ln z, \quad \chi(z) \chi(0) \sim \ln z,
$$

and furthermore, for the weight $-\frac{1}{2} \beta-\gamma$ system, the stress energy tensor becomes

$$
T=-\frac{1}{2} \partial \phi \partial \phi+\frac{1}{2} \partial \chi \partial \chi+\frac{1}{2} \partial^{2} \chi
$$

with the same central charge $c=-1$. One can then easily see that the $V^{\mathfrak{u}(1)_{F}}$ subalgebra can be identified as generated by $\partial \phi$, $\partial \chi$, and their further derivatives, while the fields $\phi$ and $\chi$ themselves, as well as their exponentials, are not allowed in $V^{\mathfrak{u}(1)_{F}}$. That is, as a vector space,

$$
V^{\mathfrak{u}(1)_{F}} \cong \mathbb{C}\left[\left\{\partial^{n} \phi, \partial^{n} \chi \mid n \geq 1\right\}\right] .
$$

In the $\beta-\gamma$ language, the flavor holonomy deformation corresponds to the insertion of

$$
e^{-\mu \int \mathrm{d}^{2} w \beta(w) \gamma(w)}
$$

in correlators. Since $J=\beta \gamma$ corresponds to $\partial \chi$ in bosonization, we conclude that in the dual picture, the correlators are deformed according to

$$
\left\langle\ldots e^{-\mu \int d^{2} w \partial x}\right\rangle
$$

This, of course, does not affect the flat space correlators.

\section{A TASTE OF MODULARITY}

We have defined four versions of the Schur index or rather the $S^{3} \times S_{y}^{1}$ partition function. Those versions differ by a $(-1)^{2(R+r)}$ refinement and by the presence or absence of the monodromy defect. In the two-dimensional language, the four versions correspond to the four spin structures on the torus labeled by $\left(v_{1}, v_{2}\right)$. Denoting the corresponding partition function as $Z^{\left(v_{1}, v_{2}\right)}\left(\tau, a_{f}\right)$, and rewriting our previous expressions in terms of theta-functions, we have

$$
\begin{aligned}
Z^{\left(v_{1}, v_{2}\right)}\left(\tau, a_{f}\right) & =\frac{Z_{\mathrm{Cas}}^{\mathrm{f}}\left(q, a_{f}\right)}{|\mathscr{W}|} \int_{\mathbb{T}} \frac{\mathrm{d} u}{2 \pi \mathrm{i} u} \eta(\tau)^{3 r_{G}-\operatorname{dim}(G)+\frac{1}{2} \operatorname{dim}(\mathbf{R})} \\
& \times \prod_{\alpha \in \Delta \backslash\{0\}} \theta_{1}\left(\frac{\langle\alpha, a\rangle}{2 \pi} ; \tau\right) \prod_{w \in \mathbf{R}} \vartheta_{1-v_{1}, 1-v_{2}}\left(\frac{\langle w, a\rangle}{2 \pi}+\frac{\left\langle w_{f}, a_{f}\right\rangle}{2 \pi} ; \tau\right)^{-1 / 2},
\end{aligned}
$$

where $\tau$ is the complex structure on the torus, as given in (217), and we included flavor holonomies $a_{f}$. For completeness, we also included the flavor Casimir term $Z_{\text {Cas }}^{\mathrm{f}}\left(q, a_{f}\right)=q^{-\frac{1}{4} \sum_{w_{f} \in \mathrm{R}_{f}}\left\langle w_{f}, a_{f}\right\rangle^{2}}$ from (148) in front of the integral. While $Z^{(1,0)}$ and $Z^{(1,1)}$ have finite $a_{f} \rightarrow 0$ limits, the other two quantities $Z^{(0,0)}$ and $Z^{(0,1)}$ diverge as $a_{f} \rightarrow 0$, and so we keep $a_{f}$ generic and complex.

Note that $Z^{(1,0)}\left(\tau, a_{f}\right)$ corresponds to the standard Schur index, while $Z^{(1,1)}\left(\tau, a_{f}\right)$ corresponds to the "modified Schur index" as introduced by Razamat, ${ }^{51}$ and these two are related by the $T$-modular transformation, namely,

$$
Z^{(1,0)}\left(\tau \pm 1, a_{f}\right)=e^{ \pm \frac{\mathrm{i} \pi}{12}\left(2 \operatorname{dim}(G)+\frac{1}{2} \operatorname{dim}(\mathbf{R})\right)} Z^{(1,1)}\left(\tau, a_{f}\right)
$$

This relation has a clear geometric interpretation. In the spin structure $(1,0)$, a spinor is anti-periodic along $S_{\varphi}^{1}$ (call it the $A$ cycle) and periodic along $S_{y}^{1}$ (call it the $B$ cycle). Then, upon a Dehn twist along $A$-which is what the $T$-transformation is-the $B$ cycle is "replaced" by $A+B$. 
The spinor is clearly anti-periodic both along $A$ and $A+B$, which is why we obtain $Z^{(1,1)}\left(\tau, a_{f}\right)$ in this way. The four-dimensional lift of this operation is a similar large diffeomorphism on $S^{3} \times S_{y}^{1}$, i.e., we cut along $S^{3}$ at a point $\mathrm{pt} \in S_{y}^{1}$, twist the boundary by $e^{4 \pi i j_{1}}$, that is, by $\Delta \varphi=\Delta \tau=2 \pi$, and then glue it back. In our four-dimensional equations, this corresponds to a shift $M \mapsto M+1$. From this geometric picture, it is obvious that the other two partition functions transform into themselves, and indeed, we explicitly find that

$$
\begin{aligned}
& Z^{(0,0)}\left(\tau+1, a_{f}\right)=e^{\frac{\mathrm{i} \pi}{6}\left(\operatorname{dim}(G)-\frac{1}{2} \operatorname{dim}(\mathbf{R})\right)} Z^{(0,0)}\left(\tau, a_{f}\right), \\
& Z^{(0,1)}\left(\tau+1, a_{f}\right)=e^{\frac{\mathrm{i} \pi}{6}\left(\operatorname{dim}(G)-\frac{1}{2} \operatorname{dim}(\mathbf{R})\right)} Z^{(0,1)}\left(\tau, a_{f}\right) .
\end{aligned}
$$

The phase factors appearing in (235)-(237) must be interpreted in terms of global gravitational anomalies, but we leave an in-depth analysis of this for future work.

Note that the way the various spin structures transform among themselves is, of course, well-known from the study of superstring oneloop amplitudes. The novel aspect here is how these properties arise from the Schur index and have a clear four-dimensional interpretation.

Similarly, we might ask how the $S$-modular transformation acts on $Z^{\left(v_{1}, v_{2}\right)}\left(\tau, a_{f}\right)$. For this, let us first slightly modify the integral formula (234). There, we integrate over the maximal torus of the gauge group, which can be thought of as

$$
\mathbb{T} \cong \mathfrak{t} / 2 \pi \Lambda_{\mathrm{ch}}^{\vee},
$$

with $\mathfrak{t}$ being the Cartan subalgebra and $\Lambda_{\mathrm{ch}}^{\vee}$ being the cocharacter lattice of the gauge group G. We may complexify $\mathbb{T}$ by using the same complex structure as on the spacetime torus, i.e.,

$$
\mathbb{T}_{\mathbb{C}} \cong \mathfrak{t}_{\mathbb{C}} /\left(2 \pi \Lambda_{\mathrm{ch}}^{\vee}+2 \pi \tau \Lambda_{\mathrm{ch}}^{\vee}\right) \cong \mathbb{T} \oplus \tau \mathbb{T},
$$

and consider a family of middle-dimensional cycles in $\mathbb{T}_{\mathbb{C}}$ parameterized by two integers $(m, n)$,

$$
\mathbb{T}_{(m, n)}=m \mathbb{T} \oplus n \tau \mathbb{T} .
$$

This notation means that $\mathbb{T}_{(m, n)}$ wraps the original real cycle $\mathbb{T}$ exactly $m$ times and $n$ times the "imaginary" cycle $\tau \mathbb{T}$. This is, of course, a higher-dimensional analog of an $m A+n B$ cycle on a two-torus.

In Eq. (234), $u=e^{\mathrm{i} a}$, and we integrate $a$ over the real cycle $\mathbb{T}_{(1,0)}$. We may consider an integral over a more general cycle and define the following quantity: 10

$$
\begin{aligned}
Z_{(m, n)}^{\left(v_{1}, v_{2}\right)}\left(\tau, a_{f}\right)= & \frac{Z_{\mathrm{Cas}}^{\mathrm{f}}\left(q, a_{f}\right)}{|\mathscr{W}|} \int_{\mathbb{T}_{(m, n)} \subset \mathfrak{t}_{\mathbb{C}} /\left(2 \pi \Lambda_{\mathrm{ch}}^{\vee}+2 \pi \tau \Lambda_{\mathrm{ch}}^{\vee}\right)} \frac{\mathrm{d} a}{2 \pi} \eta(\tau)^{3 r_{G}-\operatorname{dim}(G)+\frac{1}{2} \operatorname{dim}(\mathbf{R})} \times \\
& \times \prod_{\alpha \in \Delta \backslash\{0\}} \theta_{1}\left(\frac{\langle\alpha, a\rangle}{2 \pi} ; \tau\right) \prod_{w \in \mathbf{R}} \vartheta_{1-v_{1}, 1-v_{2}}\left(\frac{\langle w, a\rangle}{2 \pi}+\frac{\left\langle w_{f}, a_{f}\right\rangle}{2 \pi} ; \tau\right)^{-1 / 2}
\end{aligned}
$$

such that the original integral (234) is $Z_{(1,0)}^{\left(v_{1}, v_{2}\right)}\left(\tau, a_{f}\right)$. A straightforward computation shows

$$
Z_{(m, n)}^{\left(v_{1}, v_{2}\right)}\left(-\frac{1}{\tau}, \frac{a_{f}}{\tau}\right)=e^{-\frac{\mathrm{i} \pi}{2}\left(\operatorname{dim}(G)-\frac{1}{2} \operatorname{dim}(\mathbf{R}) \delta_{v_{1}, 0}, 0 \delta_{v_{2}, 0}\right)} Z_{(-n, m)}^{\left(v_{2}, v_{1}\right)}\left(\tau, a_{f}\right) e^{\frac{i \pi}{2}\left(\frac{1}{\tau^{3}}+\frac{1}{2 \pi^{2} \tau}+\tau\right) \sum_{w f}\left(w_{f}, a_{f}\right\rangle^{2}} .
$$

Again, we postpone explanation of the overall phase for future work. We see that the $S$-transformation both flips the spin structure, $\left(v_{1}, v_{2}\right)$ $\mapsto\left(v_{2}, v_{1}\right)$ (as expected), and changes the integration cycle, $(m, n) \mapsto(-n, m)$. In particular, the original cycle $(1,0)$ goes to $(0,1)$, which is not equivalent to the $(1,0)$-cycle.

We can also check how the more general object $Z_{(m, n)}^{\left(v_{1}, v_{2}\right)}\left(\tau, a_{f}\right)$ transforms under the $T$-modular transformation, generalizing the case of $(m, n)=(1,0)$ described at the beginning of this section. We explicitly find that

$$
Z_{(m, n)}^{\left(v_{1}, v_{2}\right)}\left(\tau+1, a_{f}\right)=e^{\frac{i \pi}{6}\left(\operatorname{dim}(G)-\frac{1}{2} \operatorname{dim}(\mathbf{R})+\frac{3}{4} \operatorname{dim}(R) \delta_{v_{1}, 1}\right)} Z_{(m+n, n)}^{\left(v_{1}, v_{2}+v_{1}\right)}\left(\tau, a_{f}\right),
$$

where $v_{1}+v_{2}$ should be taken as a remainder modulo 2 , i.e., $1+1$ is replaced by 0 .

To summarize, we have found that the functions $Z_{(m, n)}^{\left(v_{1}, v_{2}\right)}\left(\tau, a_{f}\right)$ transform in an interesting way under the modular group. It permutes their discrete labels $(m, n)$ according to the $S L(2, \mathbb{Z})$ action on the lattice $\mathbb{Z}^{2}$. As for the $\mathbb{Z}_{2} \times \mathbb{Z}_{2}$ labels $\left(v_{1}, v_{2}\right)$, the $(0,0) \in \mathbb{Z}_{2} \times \mathbb{Z}_{2}$ is fixed, while the other three, $(0,1),(1,0),(1,1)$, are permuted by $S L(2, \mathbb{Z})$.

The transformation property (242) involves a cumbersome $a_{f}$-dependent exponential factor, which we would like to remove by taking the $a_{f} \rightarrow 0$ limit. This limit is also interesting due to the relation with Ref. 26. However, as we know, this limit might be singular. More 
precisely, it is singular for those integration contours $\mathbb{T}_{(m, n)}$ that pass through the poles of the integrand of $(241)$ as $a_{f} \rightarrow 0$. It turns out that this property is preserved by the modular group, and the following holds:

An observation. If $Z_{(m, n)}^{\left(v_{1}, v_{2}\right)}\left(\tau, a_{f}\right)$ has a finite $a_{f} \rightarrow 0$ limit, then so do its images under the $S L(2, \mathbb{Z})$ action described above.

This simple observation implies that for a given $\left(v_{1}, v_{2}\right)$, there is a set $\Sigma^{\left(v_{1}, v_{2}\right)}$ of integration cycles $\mathbb{T}_{(m, n)}$ that admit a well-defined $a_{f} \rightarrow 0$ limit. Let us denote the corresponding limits as

$$
Z_{(m, n)}^{v_{1}, v_{2}}(\tau)=\lim _{a_{f} \rightarrow 0} Z_{(m, n)}^{\left(v_{1}, v_{2}\right)}\left(\tau, a_{f}\right)
$$

whose modular transformations follow trivially from (242) and (243). Note that for $Z_{(m, n)}^{(0,0)}\left(\tau, a_{f}\right)$, there does not exist a tuple of integers, $(m, n)$, which renders the limit $a_{f} \rightarrow 0$ regular. This is because there is a pole at $a=0$ in the respective integrand. Nevertheless, for the other three spin structures, there are many such integers $(m, n)$ with a finite answer in the $a_{f} \rightarrow 0$ limit. Thus, we find a triplet of $\mathbb{Z}^{2}$-labeled functions,

$$
\left(Z_{(m, n)}^{(0,1)}(\tau), Z_{(m, n)}^{(1,0)}(\tau), Z_{(m, n)}^{(1,1)}(\tau)\right)
$$

where each function has its own set $\Sigma^{\left(v_{1}, v_{2}\right)}$ of allowed $(m, n) \in \Sigma^{\left(v_{1}, v_{2}\right)}$ labels. The modular group permutes them, and thus, up to phases, they all transform as the components of a weight- 0 modular vector. In particular, the standard Schur index $Z_{(1,0)}^{(1,0)}(\tau)$ belongs to this family, and so do its $T$-transform $Z_{(1,0)}^{(1,1)}(\tau)$ and its $S$-transform $Z_{(0,1)}^{(0,1)}(\tau)$.

What we described so far looks like an infinite-dimensional projective representation of $S L(2, \mathbb{Z})$. In concrete physical theories, it is expected to truncate. Specifically, not all functions $Z_{(m, n)}^{\left(v_{1}, v_{2}\right)}(\tau)$ will be independent, and a (possibly finite) set of independent functions will transform as some modular vector. ${ }^{26}$

Finally, physically, we expect that the quantities $Z_{(m, n)}^{\left(v_{1}, v_{2}\right)}\left(\tau, a_{f}\right)$, defined as integrals over the non-standard cycles $\mathbb{T}_{(m, n)}$, correspond to partition functions in the presence of more general defects, i.e., characters of some non-trivial modules of the VOA. Those functions that are singular in the limit $a_{f} \rightarrow 0$ correspond to modules that have infinite degeneracies at each energy level. Such modules are not pathological, and in fact, we have seen above that Ramond modules of the underlying symplectic boson exhibit such a behavior, the quantities $Z_{(1,0)}^{(0,0)}\left(\tau, a_{f}\right)$ and $Z_{(1,0)}^{(0,1)}\left(\tau, a_{f}\right)$ being our basic examples of those.

Let us now briefly review a couple of simple examples.

\section{A. Examples}

\section{1. $S U(2)$ with $N_{f}=4$} given by

An $S U(2)$ gauge theory with four hypermultiplets is the simplest interacting Lagrangian superconformal field theory. Its Schur index is

$$
\begin{aligned}
Z_{(1,0)}^{(1,0)}(\tau) & =-\frac{1}{4 \pi} \int_{0}^{2 \pi} \mathrm{d} a \eta(\tau)^{8} \frac{\prod_{\alpha \in\{ \pm 1\}} \theta_{1}\left(\frac{\langle\alpha, a\rangle}{2 \pi} ; \tau\right)}{\left[\prod_{w \in\left\{ \pm \frac{1}{2}\right\}} \vartheta_{0,1}\left(\frac{\langle w, a\rangle}{2 \pi} ; \tau\right)\right]^{4}} \\
& =q^{\frac{14}{24}}\left(1+28 q+329 q^{2}+2632 q^{3}+16380 q^{4}+O\left(q^{5}\right)\right)
\end{aligned}
$$

Up to a constant phase, the $T$-transform is expressed by the same formula with $\vartheta_{0,1}$ replaced by $\vartheta_{0,0}$, and it gives the same result, i.e., we find

$$
Z_{(1,0)}^{(1,1)}(\tau)=Z_{(1,0)}^{(1,0)}(\tau)
$$

This is not surprising because the corresponding VOA is believed to be $\mathfrak{s o}(8)_{-2}$, which does not have any half-integer graded operators. Therefore, changing the spin structure from $(1,0)$ to $(1,1)$ should not affect the answer.

We can also take a look at the $S$-transform of the Schur index that, up to an overall phase factor, is given by

$$
\begin{aligned}
Z_{(0,1)}^{(0,1)}(\tau)= & -\frac{1}{4 \pi} \int_{0}^{2 \pi \tau} \mathrm{d} a \eta(\tau)^{8} \frac{\prod_{\alpha \in\{ \pm 1\}} \theta_{1}\left(\frac{\langle\alpha, a\rangle}{2 \pi} ; \tau\right)}{\left[\prod_{w \in\left\{ \pm \frac{1}{2}\right\}} \vartheta_{1,0}\left(\frac{\langle w, a\rangle}{2 \pi} ; \tau\right)\right]^{4}} \\
= & \frac{q^{\frac{14}{24}}}{120 \pi}\left(\frac{1}{q}+250+60 \log (q)+(4625+1680 \log (q)) q\right. \\
& \left.+(44250+19740 \log (q)) q^{2}+(305750+157920 \log (q)) q^{3}+\ldots\right) .
\end{aligned}
$$


The logarithmic pieces appearing in this expression might look surprising; however, such a behavior was already announced in Ref. 26. Additionally, the leading order term $\frac{1}{q}$ also agrees with their results. We leave the interpretation of this phenomenon for future work.

\section{2. $\mathcal{N}=4$ super Yang-Mills with $G=S U(2)$}

Here, we consider another illustrative example, the $\mathscr{N}=4 \mathrm{SYM}$ with gauge group $S U(2)$. The Schur index is computed by

$$
\begin{aligned}
Z_{(1,0)}^{(1,0)}(\tau) & =-\frac{1}{4 \pi} \int_{0}^{2 \pi} \mathrm{d} a \eta(\tau)^{3} \frac{\prod_{\alpha \in\{ \pm 1\}} \theta_{1}\left(\frac{\langle\alpha, a\rangle}{2 \pi} ; \tau\right)}{\prod_{w \in\{-1,0,+1\}} \vartheta_{0,1}\left(\frac{\langle w, a\rangle}{2 \pi} ; \tau\right)} \\
& =q^{\frac{9}{24}}\left(1+3 q+4 q^{3 / 2}+9 q^{2}+12 q^{5 / 2}+22 q^{3}+36 q^{7 / 2}+60 q^{4}+88 q^{9 / 2}+O\left(q^{5}\right)\right) .
\end{aligned}
$$

In this case, since there are half-integral operators in the spectrum, the $T$-transformed Schur index has alternating signs, i.e.,

$$
Z_{(1,0)}^{(1,1)}(\tau)=q^{\frac{9}{24}}\left(1+3 q-4 q^{3 / 2}+9 q^{2}-12 q^{5 / 2}+22 q^{3}-36 q^{7 / 2}+60 q^{4}-88 q^{9 / 2}+O\left(q^{5}\right)\right)
$$

Similarly, we can also compute the $S$-transformed Schur index and find

$$
\begin{aligned}
Z_{(0,1)}^{(0,1)}(\tau)= & -\frac{1}{4 \pi} \int_{0}^{2 \pi \tau} \mathrm{d} a \eta(\tau)^{3} \frac{\prod_{\alpha \in\{ \pm 1\}} \theta_{1}\left(\frac{\langle\alpha, a\rangle}{2 \pi} ; \tau\right)}{\prod_{w \in\{-1,0,+1\}} \vartheta_{1,0}\left(\frac{\langle w, a\rangle}{2 \pi} ; \tau\right)} \\
= & \frac{q^{\frac{9}{24}}}{8 \pi} \times q^{-3 / 8}\left(2+\log (q)+(24+12 \log (q)) q+(24+36 \log (q)) q^{2}\right. \\
& \left.+(192+128 \log (q)) q^{3}+(152+324 \log (q)) q^{4}+O\left(q^{5}\right)\right) .
\end{aligned}
$$

Again, this is in agreement with Ref. 26, and an explanation of the logarithmic behavior calls for further investigation.

\section{RELATION TO THE $\Omega$-BACKGROUND $\mathbb{R}_{\varepsilon}^{2} \oplus \mathbb{R}^{2}$}

We now switch gears and discuss another application of our $S^{3} \times S^{1}$ background. In particular, it has long been argued ${ }^{55}$ (see also Refs. 40 and 56) that the two-dimensional chiral algebra should be obtained from putting a four-dimensional superconformal field theory on an $\Omega$-deformed background ${ }^{3,4}$ while imposing a holomorphic-topological ("Kapustin") twist. ${ }^{57,111}$ Intuitively, it is clear that this ought to be related to some "local" version of the $S^{3} \times S^{1}$ background treated in our paper, and by expanding the latter, we will explicitly see the corresponding (flat) $\Omega$-background of geometry $\mathbb{R}_{\varepsilon}^{2} \oplus \mathbb{R}^{2}$ in the following. The four-dimensional theory, of course, localizes to the tip of the cigar/origin of $\mathbb{R}_{\varepsilon}^{2}$, and the theory thus effectively reduces to the two-dimensional chiral algebra, in the same way as discussed in Secs. II and III for $S^{3} \times S^{1}$. For simplicity, we start by setting

$$
\alpha=\zeta=0
$$

and so $S^{3} \times S^{1}$ has the standard product metric, and no background R-symmetry holonomies are present.

The Killing vector arising from the Killing spinors in (74) (with $\alpha=\zeta=0$ ) is given by

$$
K^{\mu} \partial_{\mu}=-\frac{1}{2} \xi \sigma^{\mu} \bar{\xi} \partial_{\mu}=-\frac{\mathrm{i}}{\ell} \partial_{\tau}
$$

This Killing vector naturally defines a $U(1)$-action with respect to which we can define the $\Omega$-background. In this vein, we now define

$$
\varepsilon=\frac{1}{\ell}
$$

and introduce the new (complex) coordinates

$$
x=\rho e^{\mathrm{i} \tau}, \quad \bar{x}=\rho e^{-\mathrm{i} \tau},
$$

where we conveniently defined

$$
\rho=\frac{1}{\varepsilon}\left(\frac{\pi}{2}-\theta\right)
$$

which is valued in $\rho \in\left[0, \frac{\pi}{2 \varepsilon}\right] .112$ The coordinates $x$ and $\bar{x}$ parameterize directions orthogonal to the chiral algebra plane, which we recall is located at $\theta=\pi / 2$ and spanned by the coordinates $(y, \varphi)$. We can then rewrite the Killing vector $K$ in the more suggestive form 


$$
K^{\mu} \partial_{\mu}=\frac{\mathrm{i} \varepsilon}{2}\left(x \partial_{x}-\bar{x} \partial_{\bar{x}}\right)
$$

We then recall that our supercharge $\mathscr{Q}^{H}$ squares to

$$
\left(\mathscr{Q}^{H}\right)^{2} \equiv\left\{\mathbb{Q}_{1}, \mathbb{Q}_{2}\right\}=\mathrm{i} \mathscr{L}_{K}+r
$$

where $\mathscr{L}_{K}$ is the Lie derivative with respect to the vector field $K$, and $r$ is the $U(1)_{r}$-symmetry generator. This equation is inherited from the $S^{3} \times S^{1}$ background.

As we want to end up with the non-compact space $\mathbb{R}_{\varepsilon}^{2} \oplus \mathbb{R}^{2}$-or alternatively with the local version of $S^{3} \times S^{1}$ around $\theta=\frac{\pi}{2}(\rho=0)$-we ought to expand our metric in the large $\ell \rightarrow \infty$-or small $\varepsilon \rightarrow 0$-limit. To decompactify the $\varphi$ direction, we rescale

$$
\varphi \rightarrow \widehat{\varphi}=\ell \varphi
$$

and our $S^{3} \times S^{1}$-metric, written in terms of $\rho$ in (256), $\varepsilon$ in (254), and $\widehat{\varphi}$ in (259), reads

$$
\mathrm{d} s^{2}=\left[\mathrm{d} y^{2}+\mathrm{d} \widehat{\varphi}^{2}\right]+\left[\mathrm{d} \rho^{2}+\rho^{2} \mathrm{~d} \tau^{2}\right]+o\left(\varepsilon^{2}\right)
$$

corresponding to the flat space $\mathbb{R}_{\varepsilon}^{2} \oplus \mathbb{R}^{2}$.

Now, given our explicit expansion in terms of $\varepsilon$, we can write down the corresponding (flat space) Killing spinors (we refer to Appendix $\mathrm{E}$ for details) and proceed analogously to the $S^{3} \times S^{1}$ analysis performed in the earlier parts of this paper. We can explicitly check that the supersymmetry algebra still closes appropriately, solve the BPS equations (which imply the gradient flow equations mentioned in Refs. 44 and 45 ), show that the hypermultiplet action reduces to the symplectic boson action in two (flat) dimensions, and the Yang-Mills action remains $\mathscr{Q}^{H}$-exact.

Note that the supercharge we obtain in the $\varepsilon \rightarrow 0$ expansion is simply the flat space supercharge $\mathscr{Q}_{-}^{1}-\widetilde{\mathscr{Q}}_{2-}+\varepsilon\left(\mathscr{S}_{1}^{-}+\widetilde{\mathscr{S}}^{2-}\right)$, and the invariant action does not contain $\varepsilon$-it is the usual superconformal action in flat space. This corresponds to the statement in Ref. 44 that for a superconformal field theory in flat space, the relevant $\Omega$-deformation does not change the action but rather simply instructs to study the cohomology of the corresponding " $\mathscr{Q}+\mathscr{S}$ " supercharge.

\section{DISCUSSION AND OPEN QUESTIONS}

In this paper, we discussed a variety of aspects of four-dimensional superconformal field theories on the $S^{3} \times S^{1}$ background and their relation to the two-dimensional chiral algebra on the torus $S_{\varphi}^{1} \times S_{y}^{1}$. The main tool for our study was supersymmetric localization. We started by analyzing the relevant (rigid) supersymmetric background and studied the preserved subalgebras of the four-dimensional superconformal algebra. By doing so, we classified the fugacities one may turn on while still preserving the two-dimensional chiral algebra structure. Indeed, we found that, in addition to the "Schur" fugacity, we may turn on quantized (non-vanishing) fugacities $\zeta$ and $\alpha$. Although we argued that they do not affect the class of operators we count, they affect the complex structure and spin structure of the chiral algebra torus $S_{\varphi}^{1} \times S_{y}^{1}$. This naturally leads us to the discussion of the relevance of the torus spin structure and its relation to the $S^{3} \times S^{1}$ partition function. In particular, a thorough treatment of the latter requires the introduction of what we call the "canonical" R-symmetry interface, which might end on a surface defect responsible for the change in spin structure along the $\varphi$ direction. We were able to localize (in some detail) the four-dimensional theory (with the inclusion of the fugacities $\zeta$ and $\alpha$ as well as the R-symmetry defect) to arrive at the relevant two-dimensional chiral algebra quantities. Indeed, by doing so, we explained the appearance of the different spin structures and characters in two-dimensions from a four-dimensional perspective, which is intimately related to the modularity properties of the four-dimensional superconformal index. We further discussed various detailed properties of the two-dimensional chiral algebras arising from this construction and made remarks on an expansion that allows us to derive the flat $\Omega$-background underlying the chiral algebra.

Let us now briefly remark on some open questions and future directions.

One obvious, but interesting future direction involves understanding the modular properties of the Schur index for Lagrangian theories using the technology introduced in Sec. V, in particular, this involves understanding the relation of functions $Z_{(m, n)}^{\left(v_{1}, v_{2}\right)}\left(\tau, a_{f}\right)$ to surface defects, as well as their place in the story of Ref. 26, as well as related puzzles.

With the localization of the chiral algebra in hand, it is also natural to ask whether there are extensions to other geometries. For instance, the lens index ${ }^{85,113-115}$ would be a natural place to start. The results in Ref. 29 suggest that some data of the chiral algebra are stored in it. Similarly, the extensions discussed in Ref. 16 seem to be related to some slightly modified geometry, which allows for a study using localization. By turning on background fields (and thus implementing an appropriate twist), it should be straightforward to extend the rigid $\Omega$-background in Sec. VI to a curved space of the form $\mathscr{C} \times \Sigma$, where $\mathscr{C}$ and $\Sigma$ are two Riemann surfaces (this was very recently done in Refs. 44 and 45 ). This leads to the generalizations of Schur indices/characters to higher-genus Riemann surfaces $\mathscr{C}$, with the natural questions arising almost immediately: how do these quantities count Schur operators, what can they tell us about the VOA structure, and of course how they behave under the mapping class group action of $\mathscr{C}$. 
Next, the inclusion of various types of (non-local) defects, whose relation to the VOA was studied from a variety of perspectives in Refs. 18, 23, 28, 37, and 54, deserves a study from the point of view of localization on $S^{3} \times S^{1}$. In this paper, we have introduced the novel type of R-symmetry surface defects and studied their simplest version for Lagrangian theories in relation to the chiral algebra. Extending this analysis to more general defects of this type as well as other defects is a next logical step. Similarly, it might be interesting to study similar defects in the full superconformal index.

At this point, it is widely accepted that one may define the Schur index for non-conformal theories (see, e.g., Ref. 12 and 116). Similarly, there are constructions in three dimensions (see, e.g., Ref. 117), which suggest that one may relax the condition of theory being conformal in deriving the VOA. These results are very suggestive that there is a way to move away from the "conformality" of the four-dimensional theory, and it might be feasible to find a simple modification of our construction reproducing the VOA in such a more general setting. In fact, Oh and Yagi $^{44}$ proposed a possible way to achieve this by introducing anomalous surface defects and adding a certain rotational symmetry breaking term in the action, though this proposal calls for further clarification.

It is, of course, intriguing to study similar constructions (i.e., preserving some interesting subalgebra) in other dimensions, such as Refs. 50 and 118-121. Even in the three-dimensional $\mathscr{N}=4$ case, which has been scrutinized for Lagrangian theories in Refs. 50, 75, and 122 , there is no known result on extended objects and modules of the corresponding associative algebra yet. Furthermore, there is a known connection, on the one hand, between the three-sphere partition function and the quantized Coulomb and Higgs branches. ${ }^{50,75,122}$ On the other hand, there is a similar story relating three-dimensional $\mathscr{N}=4$ theories on a space of the form $\mathbb{R} \times \mathbb{R}_{\varepsilon}^{2}$, where the latter factor is the $\Omega$-background. ${ }^{120,123-126}$ Roughly speaking, the latter construction corresponds to a "local" version of the former, akin to the logic in Sec. VI. Indeed, here, we treat the ("uplifted") version in four dimensions and thus expect a three-dimensional analysis to be straightforward. In this spirit, we remark that the role of short star-products related to the distinguished basis in the associative algebra ${ }^{120}$ has never been discussed from the $\Omega$-deformation viewpoint, though the results of Ref. 122 suggest that the defining properties of such products largely constrain the algebra.

Finally, it would be very interesting to understand whether there exists a "four-dimensional mirror" version of the VOA construction. In three dimensions, this was obviously the case; the two constructions producing special "short" quantizations of the Higgs and Coulomb branches ${ }^{50,75,122}$ had connections to the mathematical construction of symplectic duality. ${ }^{124}$ It is also understood that the VOA in four dimensions is closely related to the Higgs branch of the theory, ${ }^{26,43}$ although there is intriguing evidence that it retains data about the Coulomb branch as well. . $2,27,127-129$ Understanding whether there exists some sort of "four-dimensional mirror" counterpart of the VOA naturally attached to the Coulomb sector would be a very exciting development.

Note added in proof. During the final stages of this project, the paper ${ }^{42}$ appeared, which has partial overlap with some results in our work. Additionally, the recent papers ${ }^{44,45}$ have some overlap with our Sec. VI.

\section{ACKNOWLEDGMENTS}

We thank Thomas T. Dumitrescu, Sergei Gukov, Shu-Heng Shao, Nikita Sopenko, Lev Spodyneiko, and Yifan Wang for discussions and correspondence. M.D. was supported by the Walter Burke Institute for Theoretical Physics and the U.S. Department of Energy, Office of Science, Office of High Energy Physics (Award No. de-sc0011632) as well as the Sherman Fairchild Foundation. M.F. was partially supported by the JSPS Grants-in-Aid for Scientific Research Wakate(A) (Grant No. 17H04837), the WPI Initiative, MEXT, Japan, at IPMU, the University of Tokyo, the David and Ellen Lee Postdoctoral Scholarship, and the U.S. Department of Energy, Office of Science, Office of High Energy Physics (Award No. de-sc0011632).

\section{APPENDIX A: NOTATIONS AND CONVENTIONS}

In this appendix, we introduce the relevant notations and conventions. We follow the conventions of Ref. 67 but recall them here for the convenience of the reader. We denote by $\alpha, \beta, \ldots=1,2$ and $\dot{\alpha}, \dot{\beta}, \ldots=1,2$ chiral and anti-chiral spinors $\psi_{\alpha}$ and $\bar{\psi}^{\dot{\alpha}}$, respectively. Those indices are raised and lowered by multiplying with the totally anti-symmetric tensor $\varepsilon$ in the NW-SE and SW-NE convention (i.e., upper left indices contract with lower right indices),

$$
\psi^{\alpha}=\varepsilon^{\alpha \beta} \psi_{\beta}, \quad \bar{\psi}_{\dot{\alpha}}=\varepsilon_{\dot{\alpha} \dot{\beta}} \bar{\psi}^{\dot{\beta}},
$$

where we take $\varepsilon$ to be defined via $\varepsilon^{12}=-\varepsilon_{12}=\varepsilon^{i \dot{2}}=-\varepsilon_{12}=1$. Furthermore, spinors are contracted as

$$
\psi \chi=\psi^{\alpha} \chi_{\alpha}, \quad \bar{\psi} \bar{\chi}=\bar{\psi} \dot{\alpha} \bar{\chi}^{\dot{\alpha}} .
$$

Additionally, the doublet-indices $A, B, \ldots=1,2$ are raised and lowered with $\varepsilon^{A B}$ and $\varepsilon_{A B}$, with $\varepsilon^{12}=-\varepsilon_{12}=1$, with $\xi^{A}=\varepsilon^{A B} \xi_{B}$, as well as $\xi_{A}=\varepsilon_{A B} \xi^{B}$. Finally, throughout this paper, we identify the auxiliary indices (usually denoted by $\left.\check{A}, \check{B}, \ldots\right)$ with the $S U(2)_{R}$ doublet indices $A, B, \ldots$ and thus remove the checks.

The four-dimensional gamma matrices can be decomposed into two $2 \times 2$ blocks for which we use the following choice: ${ }^{130}$

$$
\left(\sigma^{a}\right)_{\alpha \dot{\alpha}}=(-\mathrm{i} \vec{\sigma}, \mathbb{1}), \quad\left(\bar{\sigma}^{a}\right)^{\dot{\alpha} \alpha}=(\mathrm{i} \vec{\sigma}, \mathbb{1}),
$$


with $\tau^{a}$ being the standard Pauli matrices

$$
\tau^{1}=\left(\begin{array}{ll}
0 & 1 \\
1 & 0
\end{array}\right), \quad \tau^{2}=\left(\begin{array}{cc}
0 & -\mathrm{i} \\
\mathrm{i} & 0
\end{array}\right), \quad \tau^{3}=\left(\begin{array}{cc}
1 & 0 \\
0 & -1
\end{array}\right) .
$$

Furthermore, we define the tensors as follows:

$$
\left(\sigma_{a b}\right)_{\alpha}^{\beta}=\frac{1}{2}\left(\sigma_{a} \bar{\sigma}_{b}-\sigma_{b} \bar{\sigma}_{a}\right), \quad\left(\bar{\sigma}_{a b}\right)_{\dot{\beta}}^{\dot{\alpha}}=\frac{1}{2}\left(\bar{\sigma}_{a} \sigma_{b}-\bar{\sigma}_{b} \sigma_{a}\right)
$$

and correspondingly use the shorthand for the spinors $\xi_{A} \equiv \xi_{A \alpha}$ as well as $\bar{\xi}_{A} \equiv \bar{\xi}_{A}{ }^{\dot{\alpha}}$.

For the group theory notations, we use $r_{G}$ to denote the rank of $G, \Delta_{+}$to denote positive roots, and $\Delta$ to denote the whole root system, including $r_{G}$ copies of a zero root.

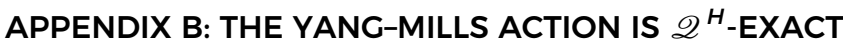

In this appendix, we show that the $\mathscr{N}=2$ Yang-Mills Lagrangian as given in Eq. (39) can be written as a $\mathscr{Q}^{H}$-exact piece (plus some surface terms). In fact, it is even possible to turn on $\alpha=2 \pi N / \beta$ and $\zeta=2 \pi M / \beta$, with $M, N \in \mathbb{Z}$. An involved yet explicit calculation shows ${ }^{131}$

$$
\begin{aligned}
& \mathscr{L}_{\mathrm{YM}} \widehat{\operatorname{vol}}_{S^{3} \times S^{1}}= \\
& \mathscr{Q}^{H}\left[-\frac{2 \mathrm{i}}{\bar{S}} \bar{\phi}\left(\left(\xi^{\prime}\right)^{A \alpha} \lambda_{A \alpha}\right)-\left\{\frac{4}{\ell S^{2}} \phi+\frac{\mathrm{i}}{S}[\phi, \bar{\phi}]\right\}\left(\xi^{A \alpha} \lambda_{A \alpha}\right)+\frac{1}{\bar{S}}\left(\mathscr{D}_{\mu} \bar{\phi}\right)\left(\left(\sigma^{\mu}\right)^{\alpha} \bar{\xi}_{\dot{\alpha}}^{A \dot{\alpha}} \lambda_{A \alpha}\right)\right. \\
& +\frac{1}{4 S} F_{\mu \nu}\left(\lambda^{A \alpha}\left(\sigma^{\mu \nu}\right)_{\alpha}^{\beta} \xi_{A \beta}\right)+\frac{1}{2 S}\left(\xi^{A \alpha} D_{A B} \lambda_{\alpha}^{B}\right) \\
& -\frac{2 \mathrm{i}}{S} \phi\left(\left(\bar{\xi}^{\prime}\right)_{\dot{\alpha}}^{A} \bar{\lambda}_{A}^{\dot{\alpha}}\right)-\left\{\frac{4}{\ell \bar{S}^{2}} \bar{\phi}-\frac{\dot{\mathrm{i}}}{\bar{S}}[\phi, \bar{\phi}]\right\}\left(\bar{\xi}_{\dot{\alpha}}^{A} \bar{\lambda}_{A}{ }^{\dot{\alpha}}\right)+\frac{1}{S}\left(\mathscr{D}_{\mu} \phi\right)\left(\left(\bar{\sigma}^{\mu}\right)_{\dot{\alpha}}{ }^{\alpha} \xi_{\alpha}^{A} \bar{\lambda}_{A}{ }^{\dot{\alpha}}\right) \\
& \left.+\frac{1}{4 \bar{S}} F_{\mu \nu}\left(\bar{\lambda}_{A \dot{\alpha}}\left(\bar{\sigma}^{\mu \nu}\right)_{\dot{\beta}}^{\dot{\alpha}} \bar{\xi}^{A \dot{\beta}}\right)+\frac{1}{2 \bar{S}}\left(\bar{\xi}_{\dot{\alpha}}^{A} D_{A B} \bar{\lambda}^{B \dot{\alpha}}\right)\right] \widehat{\operatorname{vol}}_{S^{3} \times S^{1}} \\
& + \text { (surfaceterms), }
\end{aligned}
$$

where we used the definitions for $S$ and $\bar{S}$, which we recall here

$$
S=\xi^{A} \xi_{A}=-2 e^{\mathrm{i} \tau} \cos \theta, \quad \bar{S}=\bar{\xi}^{A} \bar{\xi}_{A}=-2 e^{-\mathrm{i} \tau} \cos \theta
$$

and where

$$
\widehat{\operatorname{vol}}_{S^{3} \times S^{1}}=\ell^{3} \sin \theta \cos \theta \mathrm{d} \theta \wedge \mathrm{d} \varphi \wedge \mathrm{d} \tau \wedge \mathrm{d} y
$$

is the four-dimensional volume form on $S^{3} \times S^{1}$.

The bosonic piece of the surface-or the total derivative-terms in the last line of (B1) can be written as follows: ${ }^{132}$

$$
\text { ( bosonic surfaceterms ) }=\mathrm{d}[(\Xi+\bar{\Xi}) \wedge F+(* \Pi)] \text {, }
$$

where $F$ is the two-form field strength (we again neglected the trace over gauge indices), and the one-forms $\Xi$ and $\bar{\Xi}$ are given by

$$
\Xi=\frac{1}{S} v \phi, \quad \bar{\Xi}_{\mu}=\frac{1}{\bar{S}} v \bar{\phi},
$$

where we recall from (101) that the one-form $v$ is given by

$$
v=4 \mathrm{i} \cos ^{2} \theta\left(\ell \mathrm{d} \tau+\frac{\zeta}{\ell} \mathrm{d} y\right) .
$$

The third one-form $\Pi$ can be written as

$$
\Pi=\frac{2}{\ell S^{2}} v \phi^{2}-\frac{2}{\ell \bar{S}^{2}} v \bar{\phi}^{2}+\frac{16 \mathrm{i}}{S \bar{S}}\left(\bar{S}^{\prime}-\frac{\mathrm{i}}{4 \ell} v\right) \phi \bar{\phi},
$$


where we introduced an additional bilinear $v^{\prime}$ as follows:

$$
v^{\prime}=-\frac{1}{2} \xi \sigma_{\mu} \bar{\xi}^{\prime}=\frac{1}{2} e^{\mathrm{i} \tau+\mathrm{i} \frac{\zeta-\alpha}{\ell} y}\left(\cos \theta \mathrm{d} \tau+\mathrm{i} \sin \theta \mathrm{d} \theta+\frac{\zeta}{\ell} \cos \theta \mathrm{d} y\right)
$$

Now, the $F$-dependent pieces in (B4) include the pieces $\Xi$ and $\bar{\Xi}$, which are vanishing at the boundary of $S^{3} \times S^{1}$ at $\theta=\pi / 2$, and hence, they will not contribute to the action. ${ }^{133}$ However, the piece dependent on $\phi \bar{\phi}$ in (B4) is not vanishing at $\theta=\pi / 2$. Nevertheless, we can remove it (together with the fermionic surface terms) by the addition of a $\mathscr{Q}^{H}$-exact piece at the $\theta=\pi / 2$ surface. In fact, upon removing the vanishing $F, \phi^{2}$, and $\bar{\phi}^{2}$ dependent pieces at the boundary, we end up with the remnant terms (recall that the $\tau$ direction degenerates at $\theta=\pi / 2$ ),

$$
\left.(\text { surfaceterms })\right|_{\theta=\pi / 2}=-4 \ell^{2}\left[\phi \bar{\phi}+\frac{\mathrm{i} \ell}{4}\left(\mathscr{Q}^{H} \phi\right)\left(\mathscr{Q}^{H} \bar{\phi}\right)\right]_{\theta=\pi / 2} \mathrm{~d} \varphi \wedge \mathrm{d} y .
$$

We recall that

$$
\left.\left(\mathscr{Q}^{H}\right)^{2} \phi\right|_{\theta=\pi / 2}=\frac{4 \mathrm{i}}{\ell} \phi,\left.\quad\left(\mathscr{Q}^{H}\right)^{2} \bar{\phi}\right|_{\theta=\pi / 2}=-\frac{4 \mathrm{i}}{\ell} \bar{\phi}
$$

and thus, (B9) is $\mathscr{Q}^{H}$-exact with

$$
\left.(\text { surfaceterms })\right|_{\theta=\pi / 2}=-\left.\frac{\mathrm{i} \ell^{3}}{2} \mathscr{Q}^{H}\left[\left(\mathscr{Q}^{H} \phi\right) \bar{\phi}-\phi\left(\mathscr{Q}^{H} \bar{\phi}\right)\right] \mathrm{d} \varphi \wedge \mathrm{d} y\right|_{\theta=\pi / 2} \text {. }
$$

\section{APPENDIX C: $\mathcal{N}=1$ DESCRIPTION OF THE $\mathcal{N}=2$ BACKGROUND}

In this appendix, we describe the embedding of the $\mathscr{N}=1$ background on $S^{3} \times S^{1}$ into our $\mathscr{N}=2$ background outlined in the main text. This allows us to import $\mathscr{N}=1$ localization results for one-loop determinants and Casimir energies of the $\mathscr{N}=2$ vector and hypermultiplets. We further describe those localization results and take the necessary Schur limit.

\section{1. $\mathcal{N}=1$ supersymmetric background}

Let us first write down the corresponding $\mathscr{N}=1$ background to then proceed and leverage the localization results of Ref. 84 for our $\mathscr{N}=2$ background. We start by defining the new coordinates

$$
\phi_{1} \equiv-\tau, \quad \phi_{2} \equiv \varphi,
$$

which brings our metric in (18) into a more compatible form with the other literature. ${ }^{84}$ Furthermore, we pick the following vierbeins:

$$
\begin{aligned}
\widehat{e}^{1} & =\mathrm{d} y \\
\widehat{e}^{2} & =\ell\left[\cos ^{2} \theta \mathrm{d} \phi_{1}+\sin ^{2} \theta \mathrm{d} \phi_{2}\right]-\zeta \cos 2 \theta \mathrm{d} y \\
\widehat{e}^{3} & =\frac{1}{2} \sin 2 \theta \sin \left(\phi_{2}+\phi_{1}\right)\left[2 \zeta \mathrm{d} y-\ell\left(\mathrm{d} \phi_{1}-\mathrm{d} \phi_{2}\right)\right]-\ell \cos \left(\phi_{1}+\phi_{2}\right) \mathrm{d} \theta \\
\widehat{e}^{4} & =\frac{1}{2} \sin 2 \theta \cos \left(\phi_{2}+\phi_{1}\right)\left[2 \zeta \mathrm{d} y-\ell\left(\mathrm{d} \phi_{1}-\mathrm{d} \phi_{2}\right)\right]+\ell \sin \left(\phi_{1}+\phi_{2}\right) \mathrm{d} \theta
\end{aligned}
$$

As we will see, this choice of orthonormal frame will lead to simplifications of some expressions down the line. (They were inspired by the choice in Ref. 159.) Of course, we are dealing with a complex (Hermitian) manifold, and in particular, picking the complex coordinates

$$
\begin{aligned}
& z_{1}=\ell \sin \theta e^{\frac{y}{\ell}-\mathrm{i}\left(\phi_{2}+\frac{\zeta}{\ell} y\right)}, \\
& z_{2}=\ell \cos \theta e^{\frac{y}{\ell}+\mathrm{i}\left(-\phi_{1}+\frac{\zeta}{\ell} y\right)},
\end{aligned}
$$

we can write our metric as follows:

$$
\mathrm{d} s^{2}=h_{\alpha \bar{\beta}} \mathrm{d} z^{\alpha} \mathrm{d} \bar{z}^{\bar{\beta}}, \quad h_{\alpha \bar{\beta}}=e^{-\frac{2 y}{\ell}}\left(\begin{array}{ll}
1 & 0 \\
0 & 1
\end{array}\right)
$$

with $h_{\alpha \bar{\beta}}$ being the complex metric. 
In order to derive the correct rigid $\mathscr{N}=1$ supersymmetric background, we take the rigid limit of new minimal supergravity and follow the analysis in Refs. 62 and 134. The bosonic fields of new minimal supergravity multiplet consist of the metric as well as two auxiliary gauge fields $A_{\mu}$ and $\widetilde{A}_{\mu}$, where the real part of the former is related to the $u(1)_{R}$ symmetry of the $\mathscr{N}=1$ theory, and the latter ought to be divergence less (it arises as the Hodge dual of a three-form), i.e., $\nabla^{\mu} \widetilde{A}_{\mu}=0$. The conditions for supersymmetry are given by the existence of a nontrivial solution to the following Killing spinor equations: ${ }^{135}$

$$
\begin{aligned}
& \left(\nabla_{\mu}-\mathrm{i} A_{\mu}\right) \zeta+\mathrm{i} \widetilde{A}_{\mu} \zeta+\mathrm{i} \widetilde{A}^{\mu} \sigma_{\mu \nu} \zeta=0, \\
& \left(\nabla_{\mu}+\mathrm{i} A_{\mu}\right) \bar{\zeta}-\mathrm{i} \widetilde{A}_{\mu} \bar{\zeta}-\mathrm{i} \widetilde{A}^{\mu} \bar{\sigma}_{\mu \nu} \bar{\zeta}=0 .
\end{aligned}
$$

Here, the covariant derivatives are defined as follows:

$$
\begin{aligned}
& \nabla_{\mu} \zeta_{\alpha}=\partial_{\mu} \zeta_{\alpha}-\frac{1}{2} \omega_{\mu}^{a b}\left(\sigma_{a b}\right)_{\alpha}^{\beta} \zeta_{\beta}, \\
& \nabla_{\mu} \bar{\zeta}^{\dot{\alpha}}=\partial_{\mu} \bar{\zeta}^{\dot{\alpha}}-\frac{1}{2} \omega_{\mu}^{a b}\left(\bar{\sigma}_{a b}\right)_{\dot{\beta}}^{\dot{\alpha}} \bar{\zeta}^{\dot{\beta}} .
\end{aligned}
$$

It was shown that the existence of nontrivial solutions to equations (C5) and (C6) is equivalent to the existence of an almost complex structure of the four-dimensional manifold. ${ }^{134}$ Thus, there exists a fundamental two-form $J_{\mu \nu}$ of type $(1,1)$, which is expressed in terms of the Killing spinors

$$
J_{\mu \nu}=\frac{\mathrm{i}}{|\zeta|^{2}} \zeta^{\dagger} \sigma_{\mu \nu} \zeta,
$$

where $\left(\zeta^{\dagger}\right)^{\alpha}=\left(\zeta^{*}\right)_{\alpha}$, with the latter being the complex conjugate. In fact, together with the complex two-form of type $(2,0)$, $\zeta \sigma_{\mu \nu} \zeta$, this generates a $U(2)$ structure. We can compute $J_{\mu \nu}$ from the metric in complex coordinates (C4) and obtain ${ }^{136}$

$$
\frac{1}{2} J_{\mu \nu} \widehat{e}^{\mu} \wedge \widehat{e}^{y}=-\widehat{e}^{1} \wedge \widehat{e}^{2}+\widehat{e}^{3} \wedge \widehat{e}^{4}
$$

where we use the frame in Eq. (C2). Now, if we further define the function

$$
s=|\zeta|^{2}=\left(\zeta^{\dagger}\right)^{\alpha} \zeta_{\alpha}
$$

we can express the background fields $V_{\mu}$ and $\widetilde{V}_{\mu}$ in terms of these data. Specifically,

$$
\begin{aligned}
& \widetilde{A}_{\mu}=-\frac{1}{2} \nabla^{\rho} J_{\rho \mu}+u_{\mu}, \\
& A_{\mu}=A_{\mu}^{\mathrm{c}}-\frac{1}{4}\left(\delta_{\mu}^{v}-\mathrm{i} J_{\mu}{ }^{v}\right) \nabla^{\rho} J_{\rho v}+\frac{3}{2} u_{\mu},
\end{aligned}
$$

where $u_{\mu}$ is an arbitrary holomorphic (i.e., $J^{\mu}{ }_{\nu} u^{v}=\mathrm{i} u^{\mu}$ ) vector field satisfying $\nabla^{\mu} u_{\mu}=0$. Furthermore, $A_{\mu}^{\mathrm{c}}$ is given by

$$
A_{\mu}^{\mathrm{c}}=\frac{1}{4} J_{\mu}{ }^{\nu} \partial_{\nu} \log h-\frac{\mathrm{i}}{2} \nabla_{\mu} \log s,
$$

with $h=\operatorname{det} h_{\alpha \bar{\beta}}$.

Now, for

$$
s=\frac{C e^{\kappa y}}{\sqrt{h}},
$$

with some arbitrary constants $C$ and $\kappa$, we find that a convenient choice for $u_{\mu}$ in (C12) and (C11) gives

$$
\widetilde{A}_{\mu} \mathrm{d} x^{\mu}=\frac{\mathrm{i}}{\ell} \mathrm{d} y, \quad u_{\mu} \mathrm{d} x^{\mu}=\frac{\mathrm{i}}{\ell}\left(\widehat{e}^{1}+\widehat{e}^{2}\right)
$$

and

$$
A_{\mu} \mathrm{d} x^{\mu}=-\frac{\mathrm{i} \kappa}{2} \mathrm{~d} y
$$

and thus-in accordance with our $\mathscr{N}=2$ background-both background fields are purely gauge holonomies for the (temporal) cycle. 
This choice of background fields allows us to go back and solve the Killing spinor equations. We find

$$
\zeta=\sqrt{C} e^{y\left(\frac{\kappa}{2}+\frac{1}{\ell}\right)}\left(\begin{array}{l}
1 \\
0
\end{array}\right), \quad \bar{\zeta}=\sqrt{\widetilde{C}} e^{-y\left(\frac{\kappa}{2}+\frac{1}{\ell}\right)}\left(\begin{array}{l}
1 \\
0
\end{array}\right)
$$

for some additional constant $\widetilde{C}$ and where we used the choice of gamma matrices in (A3). ${ }^{137}$ Now, in order to get appropriate boundary conditions of the Killing spinors upon going around the $y$-circle, i.e.,

$$
\zeta(y+\beta \ell)=e^{\frac{\beta \kappa \ell}{2}+\beta} \zeta(y), \quad \bar{\zeta}(y+\beta \ell)=e^{-\frac{\beta \kappa \ell}{2}-\beta} \bar{\zeta}(y),
$$

we $f x^{1}$

$$
\kappa=-\frac{5}{2 \ell},
$$

and thus, the background one-form gauge field $A_{\mu}$ reduces to

$$
A_{\mu} \mathrm{d} x^{\mu}=\frac{5 \mathrm{i}}{4 \ell} \mathrm{d} y
$$

With our Killing spinors $\zeta$ and $\bar{\zeta}$, we can compute the Killing vector

$$
K^{\mu} \partial_{\mu}=-\frac{1}{s^{2}} \zeta \sigma^{\mu} \bar{\zeta} \partial_{\mu}=\frac{1}{2}\left[\left(\frac{1}{\ell}-\frac{\mathrm{i} \zeta}{\ell}\right) \partial_{\phi_{1}}+\left(\frac{1}{\ell}+\frac{\mathrm{i} \zeta}{\ell}\right) \partial_{\phi_{2}}-\mathrm{i} \partial_{y}\right]
$$

Given the general expression for the Killing vector of Hopf surfaces ${ }^{84}$

$$
K=\frac{1}{2}\left(b_{1} \partial_{\phi_{1}}+b_{2} \partial_{\phi_{2}}-\mathrm{i} \partial_{y}\right)
$$

we can identify

$$
\ell b_{1}=(1-\mathrm{i} \zeta), \quad \ell b_{2}=(1+\mathrm{i} \zeta) .
$$

Let us for completeness mention that one can also use $\bar{\zeta}$ to define the two-form $\bar{J}_{\mu \nu} \propto \bar{\zeta}^{\dagger} \bar{\sigma}_{\mu \nu} \bar{\zeta}$. Analogous to the case discussed above, there are formulas that relate the geometry to the background fields, and a careful analysis shows that one ends up with the equivalent background fields.

Now, in Ref. 84, it was shown that the $\mathscr{N}=1$ partition function of Hopf surfaces only depend on the complex structure parameters $p$ and $q$. Furthermore, it was argued that these parameters are related to $b_{1}$ and $b_{2}$ appearing above as follows:

$$
p=e^{-\beta \ell b_{1}}, \quad q=e^{-\beta \ell b_{2}}
$$

where as before $\beta$ is the periodicity of $y$, i.e., $y \sim y+\beta \ell$. Thus, we end up with exactly the same $p$ and $q$ as in the $\mathscr{N}=2$ case we deal with in the main text and whose relation to our deformation parameters appears in Eqs. (14)-(16).

Alternatively, these complex structure parameters $p$ and $q$ are obtained by observing that in the complex coordinate of Eq. (C3), the identification upon going around the circle, $y \sim y+\beta \ell$, reads

$$
\left(z_{1}, z_{2}\right) \sim\left(e^{-\beta(1-\mathrm{i} \zeta)} z_{1}, e^{-\beta(1+\mathrm{i} \zeta)} z_{2}\right)
$$

and thus, one can identify $p$ and $q$ in agreement with (C24).

\section{2. $\mathscr{N}=1$ localization results}

Let us now cite the relevant $\mathscr{N}=1$ localization results, as derived in Ref. 84 . We reiterate that the partition function is only dependent on the complex structure of the four-dimensional manifold. ${ }^{134}$ In our particular case, this means that it will only depend on the parameters $p$ and $q$.

The $\mathscr{N}=1$ vector multiplet, as computed from localization, see Refs. 83 and 84 (we will use the latter results; for index-results, see Refs. 61 and 90 as well as references therein) 


$$
Z_{\text {vec }}^{(\mathcal{N}=1)}=e^{-\pi \beta \ell E_{\mathrm{v}, \mathcal{N}=1}^{(0)}}(p ; p)_{\infty}^{r_{G}}(q ; q)_{\infty}^{r_{G}} \Delta_{\mathrm{VdM}}^{-1} \prod_{\alpha \in \Delta_{+}} \theta\left(u^{\alpha}, p\right) \theta\left(u^{-\alpha}, q\right),
$$

where the Casimir energy prefactor is given by

$$
E_{\mathrm{v}, \mathcal{N}=1}^{(0)}=\frac{1}{12}\left(b_{1}+b_{2}-\frac{b_{1}+b_{2}}{b_{1} b_{2}}\right) \operatorname{dim} G-\frac{b_{1}+b_{2}}{b_{1} b_{2}} \sum_{\alpha \in \Delta_{+}}\langle\alpha, a\rangle^{2} .
$$

Furthermore, by $r_{G}$, we denote the rank of the gauge group $G$, by $\Delta_{\mathrm{VdM}}$, the Vandermonde determinant,

$$
\Delta_{\mathrm{VdM}}=\prod_{\alpha \in \Delta_{+}}\left(1-u^{\alpha}\right)\left(1-u^{-\alpha}\right),
$$

of the given gauge group, and by $\Delta_{+}$, the positive roots of $G$, and finally, we defined

$$
u^{\alpha}=e^{\mathrm{i}\langle\alpha, a\rangle},
$$

where $u=e^{\mathrm{i} a}$ is the holonomy for the gauge group $G$, and by $\langle\cdot, \cdot\rangle$, we denote the canonical pairing of the Lie algebra $\mathfrak{g}=$ Lie $G$ with its dual. In addition, $p$ and $q$ are given in terms of our parameters as in (C24). Finally, the elliptic theta function is defined as follows:

$$
\theta(z, p)=(z ; p)_{\infty}(p / z ; p)_{\infty},
$$

with the Pochhammer symbol defined as

$$
(z ; q)_{\infty}=\prod_{i \geq 0}\left(1-z q^{i}\right) .
$$

Similarly, we can write down the partition function of an $\mathscr{N}=1$ chiral multiplet in a representation $\mathscr{R}$ of the gauge group $G$ (and/or flavor symmetry group) and of $\mathscr{N}=1 U(1)_{r}$-charge $r_{\mathcal{N}=1}$,

$$
Z_{\text {mat }}^{(\mathcal{N}=1)}=e^{-\pi \beta \ell E_{\text {mat }}^{(0)} \mathscr{N}=1} \prod_{w \in \mathscr{R}} \Gamma\left(u^{w}(p q)^{\frac{r}{r_{\mathcal{W}=1}}} ; p, q\right),
$$

where we again use the shorthand $u^{w}=e^{\mathrm{i}\langle w, a\rangle}$, take the product over weights of $\mathscr{R}$, with the Casimir energy given as follows:

$$
\begin{aligned}
E_{\text {mat, } \mathscr{N}=1}^{(0)}= & \sum_{w \in \mathscr{R}} \frac{1}{b_{1} b_{2}}\left\{-\frac{\mathrm{i}}{3}\langle w, a\rangle^{3}+\left(r_{\mathcal{N}=1}-1\right) \frac{b_{1}+b_{2}}{2}\langle w, a\rangle^{2}\right. \\
& \left.+\frac{\mathrm{i}}{12}\left[3\left(r_{\mathcal{N}=1}-1\right)^{2}\left(b_{1}+b_{2}\right)^{2}-2-b_{1}^{2}-b_{2}^{2}\right]\langle w, a\rangle\right\} \\
& +\frac{b_{1}+b_{2}}{24 b_{1} b_{2}}\left[\left(r_{\mathcal{N}=1}-1\right)^{3}\left(b_{1}+b_{2}\right)^{2}-\left(r_{\mathcal{N}=1}-1\right)\left(b_{1}^{2}+b_{2}^{2}+2\right)\right] \operatorname{dim} \mathscr{R},
\end{aligned}
$$

where we sum over the weights of the representation $\mathscr{R}$. Here, we write $p$ and $q$ in terms of the squashing parameters $b_{1}$ and $b_{2}$ as in (C24). Finally, the elliptic gamma function is defined as follows:

$$
\Gamma(z, p, q)=\prod_{i, j \geq 0} \frac{1-p^{i+1} q^{i+1} / z}{1-p^{i} q^{i} z} .
$$

\section{Embedding into $\mathscr{N}=\mathbf{2}$ background}

Now let us carefully embed the above $\mathscr{N}=1$ background in new minimal supergravity into our $\mathscr{N}=2$ background in the standard Weyl multiplet detailed in the main text. As we already mentioned, the complex structure parameters $p$ and $q$, which completely determine the $\mathscr{N}=1$ partition function precisely coincide with the aptly named $\mathscr{N}=2$ parameters, $p$ and $q$. These parameters can thus be interpreted geometrically. The remaining $\mathscr{N}=2$ parameter, $t$, is related to the constant $\alpha$, which enters the background gauge fields $V$ and $\widetilde{V}$ as in Eqs. (33) and (34).

We start by recalling the $\mathscr{N}=1$ actions for the chiral and vector multiplets and then move to the study of their embedding into the $\mathscr{N}=2$ vector and hypermultiplet actions (39) and (40). 
The $\mathscr{N}=1$ vector multiplet consists of the components

$$
\left(\mathscr{A}_{\mu}, \lambda, \bar{\lambda}, D\right)
$$

with $\mathscr{A}_{\mu}$ being a gauge field, $\lambda, \bar{\lambda}$ being two spinors of opposite chirality, and $D$ being an additional auxiliary field. The fields in (C35) have assigned $\mathscr{N}=1 \mathrm{R}$-charges $(0,1,-1,0)$. Then, the $\mathscr{N}=1$ vector multiplet action reads

$$
\mathscr{L}_{\text {vec }}^{(\mathscr{N}=1)}=\operatorname{Tr}\left(\frac{1}{4} \mathscr{F}^{\mu \nu} \mathscr{F}_{\mu \nu}-\frac{1}{2} D^{2}+\frac{\mathrm{i}}{2} \lambda \sigma^{\mu} D_{\mu}^{\mathrm{cs}} \bar{\lambda}+\frac{\mathrm{i}}{2} \bar{\lambda} \bar{\sigma}^{\mu} D_{\mu}^{\mathrm{cs}} \lambda\right)
$$

with

$$
D_{\mu}^{\mathrm{cs}}=\nabla_{\mu}-\mathrm{i} \mathscr{A}_{\mu} \cdot-\mathrm{i} q_{R}\left(A_{\mu}-\frac{3}{2} \widetilde{A}_{\mu}\right)
$$

where by $q_{R}$ we denote the $\mathscr{N}=1 U(1)_{r}$ R-charge of the respective fields and by the action in the appropriate representation.

Similarly, the $\mathscr{N}=1$ chiral multiplet

$$
(\phi, \bar{\phi}, \psi, \bar{\psi}, F, \bar{F})
$$

consists of two complex scalars $\phi$ and $\bar{\phi}$, spinors $\psi$ and $\bar{\psi}$, and auxiliary fields $F$ and $\bar{F}$. The $U(1)_{R}$ charges of $($ C38) are given by $\left( \pm r_{\mathscr{N}=1}, \pm\left(r_{\mathscr{N}=1}-1\right), \pm\left(r_{\mathscr{N}=1}-2\right)\right)$. Then, the $\mathscr{N}=1$ chiral multiplet action reads

$$
\begin{aligned}
\mathscr{L}_{\text {mat }}^{(\mathscr{N}=1)}= & D_{\mu} \bar{\phi} D^{\mu} \phi+\widetilde{A}^{\mu}\left(\mathrm{i} D_{\mu} \bar{\phi} \phi-\mathrm{i} \bar{\phi} D_{\mu} \phi\right)+\frac{r_{\mathscr{N}=1}}{4}\left(R+6 \widetilde{A}_{\mu} \widetilde{A}^{\mu}\right) \bar{\phi} \phi+\bar{\phi} D \phi-\bar{F} F \\
& +\mathrm{i} \bar{\psi}^{\mu} D_{\mu} \psi+\frac{1}{2} \widetilde{A}^{\mu} \overline{\psi \sigma}_{\mu} \psi+\mathrm{i} \sqrt{2}(\bar{\phi} \lambda \psi-\bar{\psi} \bar{\lambda} \phi),
\end{aligned}
$$

where the covariant derivatives contain the $\mathscr{N}=1 u(1)_{r}$ background gauge field $A_{\mu}$ as well as the (dynamical) gauge field $\mathscr{A}_{\mu}$ in the vector multiplet, i.e.,

$$
D_{\mu}=\nabla_{\mu}-\mathrm{i} \mathscr{A}_{\mu} \cdot-\mathrm{i} q_{R} A_{\mu}
$$

with the notation understood as above.

To arrive at the required $\mathscr{N}=2$ vector multiplet action, we start with the $\mathscr{N}=1$ background introduced above and take a vector multiplet in some representation of the gauge group $G$, together with a chiral multiplet in the adjoint of $G$ and of R-charge $r_{\mathscr{N}=1}=\frac{2}{3}$, and should arrive at the required $\mathscr{N}=2$ Lagrangian. Similarly, the $\mathscr{N}=2$ (full) hypermultiplet action is obtained from two $\mathscr{N}=1$ chiral multiplets in representations $\mathscr{R}, \overline{\mathscr{R}}$, respectively. ${ }^{139}$

In the index-realization, to match to the enriched fugacity space of the $\mathscr{N}=2$ superconformal index, we are required to turn on a distinguished combination of flavor fugacities in the $\mathscr{N}=1$ index (see, e.g.,Refs. 90, 140, and 141). In particular, we define the $\mathscr{N}=1$ index (we neglect additional flavor fugacities, for simplicity, now),

$$
\mathscr{I}_{\mathscr{N}=1}(p, q, \xi)=\operatorname{Tr}_{\mathscr{H}_{S^{3}}}(-1)^{F} p^{j_{1}+j_{2}+\frac{r_{\mathcal{N}}=1}{2}} q^{j_{2}-j_{1}+\frac{r_{\mathcal{N}}=1}{2}} \xi^{\mathscr{F}}
$$

with $\operatorname{Tr}_{\mathscr{H}_{S^{3}}}$ being the trace over the $\mathscr{N}=1$ Hilbert space in radial quantization, $j_{i}$ and $F$ defined as in the main text, and $r_{\mathscr{N}=1}$ being the corresponding $\mathscr{N}=1 \mathrm{R}$-charge. Furthermore, the additional fugacity $\xi$ is chosen to match with the $\mathscr{N}=2$ fugacities $p, q$, and $t$,

$$
\xi=\left(\frac{t}{(p q)^{2 / 3}}\right),
$$

and $\mathscr{F}$ corresponds to the $\mathscr{F}$-charge, which is given in terms of $\mathscr{N}=2, S U(2)_{R} \times U(1)_{r}$ charges $R$ and $r$ as follows:

$$
\mathscr{F}=R+r .
$$

In particular, the adjoint chiral multiplet inside the $\mathscr{N}=2$ vector multiplet has charge $\mathscr{F}=-1$, and similarly, the chiral multiplets inside the $\mathscr{N}=2$ hypermultiplet have $\mathscr{F}=\frac{1}{2}$.

Now, in the localization-realization, this is translated to introducing the additional background gauge field

$$
a_{\mathrm{bg}}=\mathscr{F} a_{0} \mathrm{~d} y
$$


with $\mathscr{F}$ being the $\mathscr{F}$-charge as specified above and $a_{0}$ to be determined from a precise matching of the Lagrangians. Indeed, an explicit computation shows that the respective $\mathscr{N}=1$ actions reproduce the $\mathscr{N}=2$ vector and hypermultiplet Lagrangians in their respective backgrounds if and only if we precisely introduce the following background gauge field:

$$
a_{0}=\frac{1}{3}+\mathrm{i} \beta \alpha
$$

with $\beta=-1$ for the vector multiplet and $\beta=\frac{1}{2}$ for the hypermultiplet. As expected, this precisely corresponds to the fugacity combination in (C42).

Hence, our analysis shows that we can safely import the $\mathscr{N}=1$ localization results (C26) and (C32) to reproduce the $\mathscr{N}=2, S^{3} \times S^{1}$ localization. Our discussion and the explicit embedding of the $\mathscr{N}=1$ background into the $\mathscr{N}=2$ background implies that ${ }^{142}$

$$
\begin{aligned}
& Z_{\mathrm{vec}}^{(\mathscr{N}=2)}=e^{\beta \ell E_{\mathrm{vec}, \mathcal{N}=2}^{(0)}}(p ; p)^{r_{G}}(q ; q)^{r_{G}} \Delta_{\mathrm{VdM}}^{-1} \prod_{\alpha \in \Delta_{+}} \theta\left(u^{\alpha} ; p\right) \theta\left(u^{-\alpha} ; q\right) \Gamma\left(u^{ \pm \alpha} \xi^{-1}(p q)^{2 / 3} ; p ; q\right), \\
& Z_{\mathrm{mat}}^{(\mathscr{N}=2)}=e^{\beta \ell E_{\text {mat, } \mathscr{N}=2}^{(0)}} \prod_{w \in \mathscr{R}} \Gamma\left(u^{ \pm w} \xi^{1 / 2}(p q)^{2 / 3} ; p ; q\right),
\end{aligned}
$$

with additional (distinguished) background holonomy $\xi$ as in Eq. (C42) due to the required addition of the background gauge field $a_{\mathrm{bg}}$ [as given in (C45)] and where we fixed $r_{\mathscr{N}=1}=\frac{2}{3}$. Finally, the same procedure gives the additional Casimir energy piece as follows (see also Ref. 13):

$$
\begin{aligned}
& E_{\text {vec, } \mathscr{N}=2}^{(0)}=\frac{1+\mathrm{i} \alpha}{12}\left[12 \sum_{\delta \in \Delta_{+}}\langle\delta, a\rangle^{2}+\operatorname{dim} G\left(1+\zeta^{2}-2 \alpha^{2}-2 \mathrm{i} \alpha\right)\right], \\
& E_{\text {mat, } \mathscr{N}=2}^{(0)}=\frac{1+\mathrm{i} \alpha}{24}\left[-12 \sum_{w \in \mathscr{R}}\langle w, a\rangle^{2}+\operatorname{dim} \mathscr{R}\left(1-2 \zeta^{2}+\alpha^{2}-2 \mathrm{i} \alpha\right)\right] .
\end{aligned}
$$

Of course, all these expressions agree with the known expressions for the $\mathscr{N}=2$ superconformal index. ${ }^{13,48,59-61,140,143,144}$

\section{Schur limit}

Let us now collect the relevant formulas of the $\mathscr{N}=2$ partition function in the Schur limit $\left(q=t\right.$ and $p$ arbitrary) ${ }^{47,48}$ By doing so, we arrive at the following well-known results: ${ }^{145}$

$$
\begin{aligned}
\left.Z_{\mathrm{vec}}^{(\mathscr{N}=2)}\right|_{q=t} & =q^{\frac{\operatorname{dim} G}{12}+\sum_{\alpha \in \Delta_{+}}\langle\alpha, u\rangle^{2}} \prod_{\alpha \in \Delta}\left(q u^{\alpha} ; q\right)^{2} \\
& =q^{\frac{\operatorname{dim} G}{12}+\sum_{\alpha \in \Delta_{+}}\langle\alpha, u\rangle^{2}}(q ; q)^{2 r_{G}} \prod_{\alpha \in \Delta_{+}}\left(q u^{\alpha} ; q\right)^{2}\left(q u^{-\alpha} ; q\right)^{2}, \\
\left.Z_{\mathrm{mat}}^{(\mathscr{N}=2)}\right|_{q=t} & =q^{\frac{\operatorname{dim} \mathscr{R}}{24}-\frac{1}{2} \sum_{w \in \mathscr{R}}\langle w, a\rangle^{2}} \prod_{w \in \mathscr{R}} \frac{1}{\left(\sqrt{q} u^{w} ; q\right)\left(\sqrt{q} u^{-w} ; q\right)} \\
& =q^{\frac{\operatorname{dim} \mathbf{R}}{48}-\frac{1}{4} \sum_{w \in \mathbf{R}}\langle w, a\rangle^{2}} \prod_{w \in \mathbf{R}} \frac{1}{\left[\left(\sqrt{q} u^{w} ; q\right)\left(\sqrt{q} u^{-w} ; q\right)\right]^{1 / 2}},
\end{aligned}
$$

with the notation understood as in the rest of this appendix, and where in the last line, we have rewritten the matter contribution in terms of $\mathbf{R}=\mathscr{R} \oplus \overline{\mathscr{R}}$ so that the latter formula is applicable to half-hypermultiplets as well.

\section{APPENDIX D: THE R-SYMMETRY MONODROMY DEFECT}

Defect operators in a given theory are often defined either by coupling to the lower-dimensional quantum field theory (which upon integrating out the lower dimensional degrees of freedom leads to an alteration of fields in the higher dimensional theory) or by straight away modifying the space of fields one integrates over in the path integral. This then usually imposes some sort of conditions at the defect location. Our case of interest is going to be the surface defect of the latter kind. Even though the two types of constructions often happen to be related (see, e.g., Refs. 144, 146, and 147), we will not attempt to find an equivalent definition in terms of a "2D-4D coupled system."

Here, we would like to define a defect (located at $\{\theta=0\} \subset S^{3} \times S^{1}$ ) whose main property is that it creates the monodromy $(-1)$ for the $U(1)$ symmetry generated by $2(R+r)$. In a Lagrangian theory, all fields have $\frac{1}{2} \mathbb{Z}$-valued $R+r$ charges, and those that have half-integral $R+r$ are required to become anti-periodic along the circle that links the defect. All the vector multiplet fields have integral $R+r$, so they remain periodic, and the minimal choice consistent with full supersymmetry is to define the defect to be completely "invisible" for the vector multiplets. 
However, on the contrary, all the hypermultiplet fields have half-integral, i.e., $\left(\mathbb{Z}+\frac{1}{2}\right)$-valued, $R+r$ charges, so they become anti-periodic around the defect. Since, for the hypermultiplets, the supersymmetry variations are linear in the hypermultiplet fields, this seems naively consistent with all supersymmetry as well. The latter obviously cannot be true because the location of the defect breaks part of the isometries and thus can at most preserve half of the supersymmetry. This happens because simply stating that the fields are anti-periodic around the defect is incomplete. For example, for the scalar fields, it allows a behavior of the kind $q_{11} \sim$ const. $\times e^{i \varphi / 2}$ (for the defect sitting at $\theta=0$ ), and one can easily see that such configurations have infinite kinetic energy. Thus, in order to ensure that the action remains finite, one has to more carefully specify the behavior of the fields at the defect. This behavior, as well as possibly a boundary term in the supersymmetry variation, ends up breaking part of supersymmetry.

Now, let us consider full hypermultiplets (as opposed to half-hypermultiplets) and separate their component fields into the following four groups:

$$
\begin{array}{ll}
A=\left\{q_{11}, F_{11}, \psi_{21}, \bar{\psi}_{1}^{\mathrm{i}}\right\}, & A^{\prime}=\left\{q_{22}, F_{22}, \psi_{12}, \bar{\psi}_{2}^{2}\right\}, \\
B=\left\{q_{21}, F_{21}, \psi_{11}, \bar{\psi}_{1}^{2}\right\}, & B^{\prime}=\left\{q_{12}, F_{12}, \psi_{22}, \bar{\psi}_{2}^{\mathrm{i}}\right\},
\end{array}
$$

where the index structure is as before, i.e., $q_{A I}, F_{A I}, \psi_{\alpha I}$, and $\bar{\psi}^{\dot{\alpha}}{ }_{I}$, with $A=1,2$ being the $S U(2)_{R}$ index, $I=1,2$ being the $S U(2)_{F}$ index, $\alpha=1,2$ being the left spinorial indices, and $\dot{\alpha}=1,2$ being the right spinorial indices, while any additional flavor or gauge indices are suppressed. Note that the complex conjugates of bosons in $A$ and $B$ belong to $A^{\prime}$ and $B^{\prime}$, respectively, which is only possible in the full hypermultiplet case (as opposed to a half-hypermultiplet). Note that we also separate components of the same spinor into different groups, thus breaking the Lorentz symmetry, with the surviving generators $\sigma_{3}$ and $\bar{\sigma}_{3}$ corresponding precisely to the $U(1)_{\ell} \times U(1)_{r}$ isometry preserved by the defect sitting at $\theta=0$.

In addition, we recall a convenient complex coordinate,

$$
v=\sin \theta e^{\mathrm{i} \varphi},
$$

which in (112) was generalized to $\sin \theta e^{\mathrm{i}\left(\varphi+\zeta \frac{y}{\ell}\right)}$ for $\zeta \neq 0$. We find two consistent versions of the defect characterized by the following asymptotic behavior of the fields at $\theta=0$ :

$$
\begin{aligned}
& \text { Version I: } \quad A \text { and } A^{\prime}=\sqrt{v} \times(\text { smooth })+\sqrt{\bar{v}} \times(\text { smooth }), \\
& B=\frac{1}{\sqrt{v}} \times(\text { smooth }), \quad B^{\prime}=\frac{1}{\sqrt{\bar{v}}} \times(\text { smooth }), \\
& \text { Version II: } \quad B \text { and } B^{\prime}=\sqrt{v} \times(\text { smooth })+\sqrt{\bar{v}} \times(\text { smooth }), \\
& A=\frac{1}{\sqrt{\bar{v}}} \times(\text { smooth }), \quad A^{\prime}=\frac{1}{\sqrt{v}} \times(\text { smooth }) .
\end{aligned}
$$

It is straightforward, but somewhat tedious, to check that for such a behavior, the kinetic energy ends up finite, which is obvious for fields that vanish as $\sqrt{\theta}$ near the defect, but slightly less obvious for those that behave as $\frac{1}{\sqrt{v}}$ or $\frac{1}{\sqrt{v}}$. In fact, to ensure that the kinetic energy is finite, we are supposed to write the kinetic term for scalars $q_{A I}$ in the following form:

$$
-\frac{1}{2} q^{A I} \mathscr{D}^{\mu} \mathscr{D}_{\mu} q_{A I}
$$

which thus differs from a manifestly positive definite term $\frac{1}{2} \mathscr{D}^{\mu} q^{A I} \mathscr{D}{ }_{\mu} q_{A I}$ by a surface term supported at the defect. To show that (D5) indeed leads to a finite kinetic energy, one should excise a tubular neighborhood of radius $\varepsilon$ around the defect and expand the integrated kinetic energy in powers of $\varepsilon$, assuming the particular boundary behavior either in (D3) or in (D4). We then find that the singular term in this expansion cancels due to the angular integration.

Having shown that the defect admits field configurations with finite action means that, at least naively, it is a well-defined object in the path integral. Next, we have to show that it preserves the necessary supersymmetry (72). The first step is to show that supersymmetry variations of the hypermultiplet fields are consistent, namely, the $\theta \rightarrow 0$ behavior of the variation $\delta X$ for some field $X$ matches the $\theta \rightarrow 0$ behavior of $X$ itself. We have explicitly checked this by another tedious computation that this is indeed the case. ${ }^{148}$

Now, for each preserved supersymmetry, $Q \mathscr{L}=\nabla_{\mu} \Sigma^{\mu}$, we must determine the fate of the total derivative term in the presence of a defect. Specifically, it might produce an extra term supported at the defect, and we must either show that it is vanishing or cancel it by an additional boundary term. In our case, the defect is defined to be invisible for the vector multiplets, and so we only have to worry about the hypermultiplet action,

$$
Q \mathscr{L}_{\text {mat }}=\nabla_{\mu} \Sigma^{\mu}
$$


In the presence of the boundary conditions (D3) and (D4), the variation indeed produces a non-trivial surface term at the defect. This surface term can be canceled by the supersymmetry variation of the appropriate boundary correction term. Such boundary terms were encountered, e.g., in the construction of vortex defects on $S^{3}$ in Ref. 149. Since our defect is equivalent to the flavor vortex for a particular vorticity $(-1) \in U(1)_{F} \subset S U(2)_{F}$ (as mentioned in the main text), the results of Ref. 149, uplifted to four-dimensions, are expected to apply.

We also observe that, quite importantly, solutions to the BPS equations in (151) and (152) are consistent with Version I of the defect, while solutions in (153) and (154) are consistent with the boundary conditions for Version II. Finally, it must be obvious by now that the two versions of the defect correspond to the two Ramond modules of the symplectic boson.

\section{APPENDIX E: ON THE $\Omega$-BACKGROUND LIMIT}

We provide some more details on the $\Omega$-background discussed in Sec. VI as a particular expansion of the $S^{3} \times S^{1}$ background. As discussed there, we take the (flat) metric to be

$$
\mathrm{d} s_{\mathbb{R}_{\varepsilon}^{2} \oplus \mathbb{R}^{2}}^{2}=\mathrm{d} y^{2}+\mathrm{d} \widehat{\varphi}^{2}+\mathrm{d} \rho^{2}+\rho^{2} \mathrm{~d} \tau^{2}
$$

where $y, \widehat{\varphi} \in \mathbb{R}$ span the chiral algebra plane, while $\rho \in[0, \infty)$ and $\tau \in[0,2 \pi]$ are polar coordinates on the orthogonal plane. Next, we introduce the (somewhat inconvenient) frame

$$
e^{1}=-\mathrm{d} \rho, \quad e^{2}=\mathrm{d} \widehat{\varphi}, \quad e^{3}=\rho \mathrm{d} \tau, \quad e^{4}=\mathrm{d} y,
$$

which we find by employing our expansion for the $S^{3} \times S^{1}$-frame given in (19) (with $\zeta=0$ ).

Our expansion then immediately gives the Killing spinors (now in flat space), given by ${ }^{15}$

$$
\begin{gathered}
\xi=\frac{e^{\mathrm{i} \tau}}{\sqrt{2}}\left(\begin{array}{cc}
-1+\frac{y-\rho-\mathrm{i} \widehat{\varphi}}{2} \varepsilon & -\mathrm{i}+\frac{\mathrm{i} y+\mathrm{i} \rho+\widehat{\varphi}}{2} \varepsilon \\
-\mathrm{i}-\frac{\mathrm{i} y-\mathrm{i} \rho+\widehat{\varphi}}{2} \varepsilon & 1+\frac{y+\rho-\mathrm{i} \widehat{\varphi}}{2} \varepsilon
\end{array}\right), \\
\bar{\xi}=\frac{e^{-\frac{\mathrm{i} \tau}{2}}}{\sqrt{2}}\left(\begin{array}{cc}
1-\frac{\varepsilon(y-\rho-\mathrm{i} \widehat{\varphi})}{2} & -\mathrm{i}+\frac{\mathrm{i} y+\mathrm{i} \rho+\widehat{\varphi}}{2} \varepsilon \\
\mathrm{i}+\frac{\mathrm{i} y-\mathrm{i} \rho+\widehat{\varphi}}{2} \varepsilon & 1+\frac{y+\rho-\mathrm{i} \widehat{\varphi}}{2} \varepsilon
\end{array}\right) .
\end{gathered}
$$

It is then straightforward to check that these satisfy Eqs. (22)-(25) with

$$
\xi^{\prime}=\frac{e^{-\frac{i \tau}{2}} \varepsilon}{2 \sqrt{2}}\left(\begin{array}{cc}
-\mathrm{i} & -1 \\
-1 & \mathrm{i}
\end{array}\right), \quad \bar{\xi}^{\prime}=\frac{e^{\frac{i \tau}{2}} \varepsilon}{2 \sqrt{2}}\left(\begin{array}{cc}
\mathrm{i} & -1 \\
1 & \mathrm{i}
\end{array}\right),
$$

and all background fields turned off, i.e., $\left(V_{\mu}\right)_{A}^{B}=\widetilde{V}_{\mu}=T_{\mu \nu}=\bar{T}_{\mu v}=M=0$. In fact, these are just the flat space Killing spinors for the supercharge $\mathscr{Q}_{-}^{1}-\widetilde{\mathscr{Q}}_{2-}+\varepsilon\left(\mathscr{S}_{1}^{-}+\widetilde{\mathscr{S}}^{2-}\right)$. The leading term in the action is just the standard superconformal action in flat space.

We can immediately reproduce our results for $S^{3} \times S^{1}$. In particular, the supersymmetry algebra closes as in Eqs. (97)-(100), and similarly, for the vector multiplet, we have

$$
\begin{array}{ll}
Q^{2} A_{\mu}=\mathrm{i} v^{v} F_{v \mu}+D_{\mu} \Phi, & Q^{2} D_{A B}=\mathrm{i} v^{v} D_{v} D_{A B}+\mathrm{i}\left[\Phi, D_{A B}\right], \\
Q^{2} \phi=\mathrm{i} v^{v} D_{v} \phi+\mathrm{i}[\Phi, \phi], & Q^{2} \lambda_{A}=\mathrm{i} v^{v} D_{v} \lambda_{A}+\mathrm{i}\left[\Phi, \lambda_{A}\right]+\frac{\mathrm{i}}{4} \sigma^{\mu v} \lambda_{A} D_{\mu} v_{v}, \\
Q^{2} \bar{\phi}=\mathrm{i} v^{v} D_{v} \bar{\phi}+\mathrm{i}[\Phi, \bar{\phi}], & Q^{2} \bar{\lambda}_{A}=\mathrm{i} v^{v} D_{v} \bar{\lambda}_{A}+\mathrm{i}\left[\Phi, \bar{\lambda}_{A}\right]+\frac{\mathrm{i}}{4} \bar{\sigma}^{\mu v} \bar{\lambda}_{A} D_{\mu} v_{v},
\end{array}
$$

with

$$
\begin{aligned}
v_{m} \mathrm{~d} x^{m} & =4 \mathrm{i} \rho^{2} \varepsilon \mathrm{d} \tau, & w & =0, \\
\Phi & =4 \mathrm{i} \rho \varepsilon\left(e^{-\mathrm{i} \tau} \phi-e^{\mathrm{i} \tau} \bar{\phi}\right), & \Theta & =2 \mathrm{i} \varepsilon, \\
\check{\Theta}_{A B} & =0, & \Theta_{A B} & =0 .
\end{aligned}
$$

The hypermultiplet Lagrangian localizes to the flat space symplectic boson Lagrangian, where the (flat space) $\mathscr{Q}^{H}$-cohomology is now spanned by the fields 


$$
\begin{aligned}
Z_{I}(\varphi, y) & =\frac{1}{2}(y \varepsilon-\mathrm{i} \varepsilon \widehat{\varphi}+2) q_{1 I}-\frac{1}{2}(y \varepsilon-\mathrm{i} \varepsilon \widehat{\varphi}-2) \mathrm{i} q_{2 I} \\
& =q_{+I}+\frac{\varepsilon}{2}(y-\mathrm{i} \widehat{\varphi}) q_{-I}, \text { where } q_{ \pm I}=q_{1 I} \pm \mathrm{i} q_{2 I} .
\end{aligned}
$$

Furthermore, the $\mathscr{N}=2$ Yang-Mills Lagrangian remains $\mathscr{Q}^{H}$-exact.

\section{REFERENCES}

${ }^{1}$ N. Seiberg and E. Witten, “Monopoles, duality and chiral symmetry breaking in N = 2 supersymmetric QCD,” Nucl. Phys. B 431, 484-550 (1994); arXiv:hep-th/9408099 [hep-th].

${ }^{2}$ N. Seiberg and E. Witten, "Electric-magnetic duality, monopole condensation, and confinement in $N=2$ supersymmetric Yang-Mills theory," Nucl. Phys. B 426, 19-52 (1994); arXiv:hep-th/9407087 [hep-th].

${ }^{3}$ N. A. Nekrasov, "Seiberg-Witten prepotential from instanton counting," Adv. Theor. Math. Phys. 7, 831-864 (2004); arXiv:hep-th/0206161 [hep-th].

${ }^{4}$ N. Nekrasov and A. Okounkov, "Seiberg-Witten theory and random partitions," Prog. Math. 244, 525-596 (2006); arXiv:hep-th/0306238 [hep-th].

${ }^{\mathbf{5}}$ V. Pestun, "Localization of gauge theory on a four-sphere and supersymmetric Wilson loops," Commun. Math. Phys. 313, 71-129 (2012); arXiv:0712.2824 [hep-th].

${ }^{6}$ A. S. Losev, A. Marshakov, and N. A. Nekrasov, "Small instantons, little strings and free fermions," in From Fields to Strings: Circumnavigating Theoretical Physics, Vol. 1 (World Scientific, 2003), pp. 581-621; arXiv:hep-th/0302191 [hep-th].

${ }^{7}$ N. A. Nekrasov and S. L. Shatashvili, "Quantization of integrable systems and four dimensional gauge theories," in Proceedings of the 16th International Congress on Mathematical Physics (ICMP09), Prague, Czech Republic, August 3-8, 2009 (World Scientific, 2009), pp. 265-289; arXiv:0908.4052 [hep-th].

${ }^{8}$ N. Nekrasov and E. Witten, “The omega deformation, branes, integrability, and Liouville theory,” J. High Energy Phys. 2010(9), 092; arXiv:1002.0888 [hep-th].

${ }^{9}$ C. Beem, M. Lemos, P. Liendo, W. Peelaers, L. Rastelli, and B. C. van Rees, "Infinite chiral symmetry in four dimensions," Commun. Math. Phys. 336, 1359-1433 (2015); arXiv:1312.5344 [hep-th].

${ }^{10}$ C. Beem, W. Peelaers, L. Rastelli, and B. C. van Rees, “Chiral algebras of class S,” J. High Energy Phys. 2015(5), 020; arXiv:1408.6522 [hep-th].

${ }^{11}$ M. Lemos and W. Peelaers, “Chiral algebras for trinion theories,” J. High Energy Phys. 2015(2), 113; arXiv:1411.3252 [hep-th].

${ }^{12}$ C. Cordova and S.-H. Shao, "Schur indices, BPS particles, and Argyres-Douglas theories,” J. High Energy Phys. 2016(1), 040; arXiv:1506.00265 [hep-th].

${ }^{13}$ N. Bobev, M. Bullimore, and H.-C. Kim, “Supersymmetric Casimir energy and the anomaly polynomial,” J. High Energy Phys. 2015(9), 142; arXiv:1507.08553 [hep-th].

${ }^{14}$ P. Liendo, I. Ramirez, and J. Seo, “Stress-tensor OPE in $\mathscr{N}=2$ superconformal theories,” J. High Energy Phys. 2016(2), 019; arXiv:1509.00033 [hep-th].

${ }^{15}$ M. Lemos and P. Liendo, “ $\mathscr{N}=2$ central charge bounds from 2d chiral algebras,” J. High Energy Phys. 2016(4), 004; arXiv:1511.07449 [hep-th].

${ }^{16}$ S. Cecotti, J. Song, C. Vafa, and W. Yan, “Superconformal index, BPS monodromy and chiral algebras,” J. High Energy Phys. 2017(11), 013; arXiv:1511.01516 [hep-th].

${ }^{17}$ M. Buican and T. Nishinaka, “Conformal manifolds in four dimensions and chiral algebras," J. Phys. A: Math. Theor. 49, 465401 (2016); arXiv:1603.00887 [hep-th].

${ }^{18}$ C. Cordova, D. Gaiotto, and S.-H. Shao, “Infrared computations of defect Schur indices,” J. High Energy Phys. 2016(11), 106; arXiv:1606.08429 [hep-th].

${ }^{19}$ T. Arakawa and K. Kawasetsu, “Quasi-lisse vertex algebras and modular linear differential equations," arXiv:1610.05865 [math.QA] (2016).

${ }^{20}$ F. Bonetti and L. Rastelli, “Supersymmetric localization in $\mathrm{AdS}_{5}$ and the protected chiral algebra," J. High Energy Phys. 2018(8), 098; arXiv:1612.06514 [hep-th].

${ }^{21}$ J. Song, "MacDonald index and chiral algebra," J. High Energy Phys. 2017(8), 044; arXiv:1612.08956 [hep-th].

${ }^{22}$ L. Fredrickson, D. Pei, W. Yan, and K. Ye, “Argyres-Douglas theories, chiral algebras and wild Hitchin characters,” J. High Energy Phys. 2018(1), 150; arXiv:1701.08782 [hep-th].

${ }^{23}$ C. Cordova, D. Gaiotto, and S.-H. Shao, "Surface defects and chiral algebras," J. High Energy Phys. 2017(5), 140; arXiv:1704.01955 [hep-th].

${ }^{24}$ J. Song, D. Xie, and W. Yan, "Vertex operator algebras of Argyres-Douglas theories from M5-branes," J. High Energy Phys. 2017(12), 123; arXiv:1706.01607 [hep-th].

${ }^{25}$ M. Buican, Z. Laczko, and T. Nishinaka, “ $\mathscr{N}=2$ S-duality revisited,” J. High Energy Phys. 2017(9), 087; arXiv:1706.03797 [hep-th].

${ }^{26}$ C. Beem and L. Rastelli, "Vertex operator algebras, Higgs branches, and modular differential equations,” J. High Energy Phys. 2018(8), 114; arXiv:1707.07679 [hep-th].

${ }^{27}$ A. Neitzke and F. Yan, "Line defect Schur indices, Verlinde algebras and U(1) fixed points," J. High Energy Phys. 2017(11), 035; arXiv:1708.05323 [hep-th].

${ }^{28}$ Y. Pan and W. Peelaers, "Chiral algebras, localization and surface defects," J. High Energy Phys. 2018(2), 138; arXiv:1710.04306 [hep-th].

${ }^{29}$ M. Fluder and J. Song, "Four-dimensional lens space index from two-dimensional chiral algebra," J. High Energy Phys. 2018(7), 073; arXiv:1710.06029 [hep-th].

${ }^{30}$ M. Buican and Z. Laczko, “Nonunitary Lagrangians and unitary non-Lagrangian conformal field theories," Phys. Rev. Lett. 120, 081601 (2018); arXiv:1711.09949 [hep-th].

${ }^{31}$ J. Choi and T. Nishinaka, "On the chiral algebra of Argyres-Douglas theories and S-duality," J. High Energy Phys. 2018(4), 004; arXiv:1711.07941 [hep-th].

${ }^{32}$ T. Arakawa, "Representation theory of W-algebras and Higgs branch conjecture," in Proceedings of the International Congress of Mathematicians (ICM 2018), Rio de Janeiro, Brazil, August 1-9, 2018 (World Scientific, 2018), pp. 1261-1278; arXiv:1712.07331 [math.RT].

${ }^{33}$ V. Niarchos, "Geometry of Higgs-branch superconformal primary bundles," Phys. Rev. D 98, 065012 (2018); arXiv:1807.04296 [hep-th].

${ }^{34}$ B. Feigin and S. Gukov, "VOA $\left[M_{4}\right]$," J. Math. Phys. 61, $012302(2020)$.

${ }^{35}$ T. Creutzig, "Logarithmic W-algebras and Argyres-Douglas theories at higher rank," J. High Energy Phys. 2018(11), 188; arXiv:1809.01725 [hep-th].

${ }^{36}$ F. Bonetti, C. Meneghelli, and L. Rastelli, "VOAs labelled by complex reflection groups and 4d SCFTs,” J. High Energy Phys. 2019, 155; arXiv:1810.03612 [hep-th].

${ }^{37}$ T. Nishinaka, S. Sasa, and R.-D. Zhu, "On the correspondence between surface operators in Argyres-Douglas theories and modules of chiral algebra," J. High Energy Phys. 2019(3), 091; arXiv:1811.11772 [hep-th].

${ }^{38}$ P. Agarwal, S. Lee, and J. Song, "Vanishing OPE coefficients in 4d N = 2 SCFTs," J. High Energy Phys. 2019, 102 (2019).

${ }^{39}$ C. Beem, "Flavor symmetries and unitarity bounds in $\mathscr{N}=2$ SCFTs," Phys. Rev. Lett. 122, 241603 (2019).

${ }^{40}$ K. Costello and D. Gaiotto, “Twisted holography," arXiv:1812.09257 [hep-th] (2018).

${ }^{41}$ D. Xie and W. Yan, "W algebra, cosets and VOAs for 4d $\mathscr{N}=2$ SCFT from M5 branes," arXiv:1902.02838 [hep-th] (2019).

${ }^{42}$ Y. Pan and W. Peelaers, "Schur correlation functions on $S^{3} \times S^{1}$," J. High Energy Phys. 2019, 13 (2019).

${ }^{43}$ C. Beem, C. Meneghelli, and L. Rastelli, “Free field realizations from the Higgs branch,” J. High Energy Phys. 2019, 58 (2019).

${ }^{44} \mathrm{~J}$. Oh and J. Yagi, “Chiral algebras from $\Omega$-deformation,” J. High Energy Phys. 2019, 143 (2019). 
${ }^{45}$ S. Jeong, "SCFT/VOA correspondence via $\Omega$-deformation," J. High Energy Phys. 2019, 171 (2019).

${ }^{46}$ Note that we interchangeably use terms "the chiral algebra" and "the VOA" in this paper, not following the terminology where these are different mathematical objects.

${ }^{47}$ A. Gadde, L. Rastelli, S. S. Razamat, and W. Yan, “The 4d superconformal index from q-deformed 2d Yang-Mills," Phys. Rev. Lett. 106, 241602 (2011); arXiv:1104.3850 [hep-th].

${ }^{48}$ A. Gadde, L. Rastelli, S. S. Razamat, and W. Yan, “Gauge theories and MacDonald polynomials,” Commun. Math. Phys. 319, 147-193 (2013); arXiv:1110.3740 [hep-th].

${ }^{49}$ K. Farnsworth, M. A. Luty, and V. Prilepina, "Weyl versus conformal invariance in quantum field theory," J. High Energy Phys. 2017(10), 170; arXiv:1702.07079 [hep-th].

${ }^{50}$ M. Dedushenko, S. S. Pufu, and R. Yacoby, “A one-dimensional theory for Higgs branch operators,” J. High Energy Phys. 2018(3), 138; arXiv:1610.00740 [hep-th].

${ }^{51}$ S. S. Razamat, "On a modular property of N = 2 superconformal theories in four dimensions," J. High Energy Phys. 2012, 191; arXiv:1208.5056 [hep-th].

${ }^{52}$ V. P. Spiridonov and G. S. Vartanov, "Elliptic hypergeometric integrals and 't Hooft anomaly matching conditions," J. High Energy Phys. 2012(6), 016; arXiv:1203.5677 [hep-th].

${ }^{53}$ We refer to Sec. V for more details, including some comments on the convergence of these objects upon turning off flavor fugacities.

${ }^{54}$ C. Cordova, D. Gaiotto, and S.-H. Shao, "Surface defect indices and 2d-4d BPS states," J. High Energy Phys. 2017(12), 078; arXiv:1703.02525 [hep-th].

${ }^{55} \mathrm{E}$. Witten, private communication (2014).

${ }^{56}$ K. Costello and D. Gaiotto, "Vertex operator algebras and 3d $\mathscr{N}=4$ gauge theories," J. High Energy Phys. 2018, 18; arXiv:1804.06460 [hep-th].

${ }^{57}$ A. Kapustin, "Holomorphic reduction of N = 2 gauge theories, Wilson-'t Hooft operators, and S-duality," arXiv:hep-th/0612119 [hep-th] (2006).

${ }^{58}$ Indeed, in the three-dimensional $\mathscr{N}=4$ analog of our setup, there are alternative approaches, one relying on the $S^{3}$ localization ${ }^{50,75,122}$ and the other introducing an $\Omega$-background $\mathbb{R}_{\varepsilon}^{2} \oplus \mathbb{R},{ }^{125}$ and for superconformal $\mathscr{N}=4$ theories, there is a third approach, ${ }^{119,120}$ which is more closely related to the operator approach of Ref. 9 in four-dimensions. All of these determine a one-dimensional theory quantizing the Coulomb and Higgs-branch chiral rings.

${ }^{59} J$. Kinney, J. Maldacena, S. Minwalla, and S. Raju, “An index for 4 dimensional super conformal theories," Commun. Math. Phys. 275, 209-254 (2007); arXiv:hepth/0510251 [hep-th].

${ }^{60}$ C. Römelsberger, “Counting chiral primaries in N = 1, d = 4 superconformal field theories," Nucl. Phys. B 747, 329-353 (2006); arXiv:hep-th/0510060 [hep-th].

${ }^{61}$ C. Romelsberger, "Calculating the superconformal index and Seiberg duality," arXiv:0707.3702 [hep-th] (2007).

${ }^{62}$ G. Festuccia and N. Seiberg, "Rigid supersymmetric theories in curved superspace," J. High Energy Phys. 2011(6), 114; arXiv:1105.0689 [hep-th].

${ }^{63}$ B. de Wit, J. W. van Holten, and A. Van Proeyen, "Transformation rules of N = 2 supergravity multiplets," Nucl. Phys. B 167, 186 (1980).

${ }^{64}$ B. de Wit, J. W. van Holten, and A. Van Proeyen, "Structure of N = 2 supergravity," Nucl. Phys. B 184, 77 (1981); Erratum, 222, 516 (1983).

${ }^{65}$ B. de Wit, P. G. Lauwers, and A. Van Proeyen, "Lagrangians of N = 2 supergravity-matter systems," Nucl. Phys. B 255, 569-608 (1985).

${ }^{66}$ N. Hama and K. Hosomichi, "Seiberg-witten theories on ellipsoids," J. High Energy Phys. 2012(9), 033; arXiv:1206.6359 [hep-th].

${ }^{67}$ K. Hosomichi, “ $\mathscr{N}=2$ SUSY gauge theories on $S^{4}$," J. Phys. A: Math. Theor. 50, 443010 (2017); arXiv:1608.02962 [hep-th].

${ }^{68}$ An unconventional $S^{3} \times S^{1}$ background that would allow us to define the Schur index for non-conformal $\mathscr{N}=2$ theories is being studied in a long-awaited work. ${ }^{116}$

${ }^{69}$ Actually, these equations follow more straightforwardly directly from the supersymmetry conditions of $\eta_{A}$ and $\bar{\eta}_{A}$.

${ }^{70}$ Here, we mostly follow Ref. 67, except that we redefine their $\bar{\phi}$ to $-\bar{\phi}$ to have a more natural-looking reality condition for $\phi$.

${ }^{71}$ The auxiliary fields are necessary to the close part of the hypermultiplet supersymmetry off-shell, which is required for supersymmetric localization. Of course, it is not possible to close the full $\mathscr{N}=2$ supersymmetry for hypermultiplets with finitely many auxiliary fields.

${ }^{72}$ L. Bhardwaj and Y. Tachikawa, "Classification of 4d N = 2 gauge theories," J. High Energy Phys. 2013(12), 100; arXiv:1309.5160 [hep-th].

${ }^{73}$ Shifting $\zeta$ by $2 \pi M / \beta$ in this case again introduces a trivial factor of $e^{4 \pi i M j_{1}}=1$ in the Schur index.

${ }^{74} \mathrm{This}$ is true because the index (51) contains only fugacities for $E-R$ and $\mathscr{Z}$, and both of these symmetries commute with the supercharges $\mathbb{Q}_{i}$ and $\mathscr{Q}^{H}$.

${ }^{75}$ M. Dedushenko, Y. Fan, S. S. Pufu, and R. Yacoby, "Coulomb branch operators and mirror symmetry in three dimensions," J. High Energy Phys. 2018(4), 037; arXiv:1712.09384 [hep-th].

${ }^{76}$ In general, $\zeta=2 \pi M / \beta \neq 0$, which is our main case of interest, the induced metric on $S_{\varphi}^{1} \times S_{y}^{1}$ is not a product metric (see Sec. III C).

${ }^{77}$ We use the notation $\left(v_{1}, v_{2}\right)$ indicating that spinors pick up a phase $e^{\pi \mathrm{i} v_{1}}, e^{\pi \mathrm{i} v_{2}}$ upon going once around $S_{\varphi}^{1}, S_{y}^{1}$, respectively.

${ }^{78}$ This shows that the $R+r$ of Schur operators are always (half-)integral, and therefore, the refinement of the Schur index by $e^{2 \pi i N(R+r)}$ depends only on $N$ mod 2 for any superconformal field theory, not only the Lagrangian ones.

${ }^{79}$ Recall that the symplectic boson, i.e., the weight $-\frac{1}{2} \beta-\gamma$ system, has two distinct Ramond modules that satisfy either $\beta_{0}|0\rangle=0$ or $\gamma_{0}|0\rangle=0$.

${ }^{80}$ Recall that we work in the frame of Eq. (19) and with the gamma matrices in Eq. (A3).

${ }^{81}$ Note that here we identify the indices of the auxiliary fields and spinors with the $S U(2)_{R}$ ones. This is consistent with the choice of twisted periodicity for the spinors given in Eqs. (78) and (79).

${ }^{82}$ It might be tempting to follow the approach that works well for ellipsoids in Refs. 66 and 67 and choose $\check{\xi}_{A}=c \xi_{A}, \bar{\xi}_{A}=-c^{-1} \bar{\xi}_{A}$ on $S^{3} \times S^{1}$ as well, as done, e.g., in Ref. 42. On $S^{3} \times S^{1}$, the constraints (77) imply $c \propto e^{-\mathrm{i} \tau}$, which is not a smooth function on $S^{3}$. Such $\check{\xi}_{A}$ and $\bar{\zeta}_{A}$ would simply not be smooth sections of the appropriate spinor bundles, even though the formalism requires them to be smooth. In fact, one can check that choosing such auxiliary spinors would result in wrong BPS equations (meaning they would not have naturally expected properties). On the other hand, the choice in (78) and (79) gives perfectly smooth auxiliary spinors that produces correct BPS equations later in Sec. III.

${ }^{83}$ C. Closset and I. Shamir, "The $\mathscr{N}=1$ chiral multiplet on $T^{2} \times S^{2}$ and supersymmetric localization," J. High Energy Phys. 2014(3), 040; arXiv:1311.2430 [hep-th].

${ }^{84}$ B. Assel, D. Cassani, and D. Martelli, "Localization on Hopf surfaces," J. High Energy Phys. 2014(8), 123; arXiv:1405.5144 [hep-th].

${ }^{85}$ F. Benini, T. Nishioka, and M. Yamazaki, “4d index to 3d index and 2d TQFT," Phys.Rev. D 86, 065015 (2012); arXiv:1109.0283 [hep-th].

${ }^{86}$ M. Lemos, W. Peelaers, and L. Rastelli, “The superconformal index of class S theories of type D," J. High Energy Phys. 2014, 120; arXiv:1212.1271 [hep-th].

${ }^{87}$ O. Aharony, S. S. Razamat, N. Seiberg, and B. Willett, “3d dualities from 4d dualities," J. High Energy Phys. 2013(7), 149; arXiv:1305.3924 [hep-th].

${ }^{88} \mathrm{~L}$. Rastelli and S. S. Razamat, "The superconformal index of theories of class $\mathscr{S}$," in New Dualities of Supersymmetric Gauge Theories, edited by J. Teschner (Springer, 2016), pp. 261-305; arXiv:1412.7131 [hep-th].

${ }^{89}$ D. Martelli and J. Sparks, “The character of the supersymmetric Casimir energy," J. High Energy Phys. 2016(8), 117; arXiv:1512.02521 [hep-th].

${ }^{90}$ L. Rastelli and S. S. Razamat, "The supersymmetric index in four dimensions," J. Phys. A: Math. Theor. 50, 443013 (2017); arXiv:1608.02965 [hep-th]. 
${ }^{91}$ The notation here is standard, and we refer to Appendix C for more details.

${ }^{92}$ J. Lorenzen and D. Martelli, "Comments on the Casimir energy in supersymmetric field theories," J. High Energy Phys. 2015(7), 001; arXiv:1412.7463 [hep-th].

${ }^{93}$ B. Assel, D. Cassani, L. Di Pietro, Z. Komargodski, J. Lorenzen, and D. Martelli, “The Casimir energy in curved space and its supersymmetric counterpart," J. High Energy Phys. 2015(7), 043; arXiv:1503.05537 [hep-th].

${ }^{94}$ M. Dedushenko and S. Gukov, “A 2d (0,2) appetizer,” Phys. Rev. B 99, 066005 (2017); arXiv:1712.07659 [hep-th].

${ }^{95}$ M. Del Zotto and G. Lockhart, "Universal features of BPS strings in six-dimensional SCFTs," J. High Energy Phys. 2018(8), 173; arXiv:1804.09694 [hep-th].

${ }^{96}$ R. Eager, G. Lockhart, and E. Sharpe, "Hidden exceptional symmetry in the pure spinor superstring," Phys. Rev. D 101, 026006 (2019); arXiv:1902.09504 [hep-th].

${ }^{97} \mathrm{~V}$. Pestun, "Localization of the four-dimensional N = 4 SYM to a two-sphere and 1/8 BPS Wilson loops," J. High Energy Phys. 2012(12), 067; arXiv:0906.0638 [hep-th].

${ }^{98}$ Note that a particular "supersymmetric" is given.

${ }^{99}$ Recall that the vector multiplets are not affected, as the defect is defined to be invisible for them.

${ }^{100}$ Recall that it is enough to consider a free hypermultiplet coupled to a background flat connection for our purposes of computing the one-loop determinant.

${ }^{101}$ Because $\mathbf{R}$ is pseudoreal, even a stronger statement is true: for each $w \in \mathbf{R}$, there is $-w \in \mathbf{R}$.

${ }^{102}$ Note how the two-torus path integral does not distinguish the two Ramond modules. In order for the path integral answer to match the characters of these modules, one needs to assign appropriate opposite charges to the two Ramond vacua. In our case, this does not matter, again due to $\sum_{w \in \mathbf{R}} w=0$.

${ }^{103}$ C. Closset, T. T. Dumitrescu, G. Festuccia, and Z. Komargodski, “The geometry of supersymmetric partition functions," J. High Energy Phys. 2014(1), 124; arXiv:1309.5876 [hep-th].

${ }^{104}$ E. Witten, "A new look at the path integral of quantum mechanics," arXiv:1009.6032 [hep-th] (2010).

${ }^{105} \mathrm{E}$. Witten, "Analytic continuation of Chern-Simons theory," in Chern-Simons Gauge Theory: 20 Years After, Proceedings, Workshop, Bonn, Germany, August 3-7, 2009, AMS/IP Studies in Advanced Mathematics Vol. 50 (2011), pp. 347-446; arXiv:1001.2933 [hep-th].

${ }^{106}$ If we include background flavor holonomies $a_{f}$, they can be complex, as we mentioned previously; however, their imaginary part should be small enough to not alter the positive definiteness of (179).

${ }^{107}$ Thus, the R-R sector corresponds to $(0,0)$, R-NS to $(0,1)$, NS-R to $(1,0)$, and NS-NS to $(1,1)$. In the literature, one can sometimes find the opposite notation $(\alpha, \beta)$ $=\left(1-v_{1}, 1-v_{2}\right)$.

${ }^{108}$ This non-holomorphy has a direct counterpart in the three-dimensional case discussed in Ref. 50, where observables charged under global symmetries failed to be topological in the presence of twisted masses.

${ }^{109}$ B. L. Feigin, "Extensions of vertex algebras. Constructions and applications," Usp. Mat. Nauk 72, 131-190 (2017) [Russ. Math. Surveys 72(4), 707 (2017)].

${ }^{110}$ For now, we take this as a definition of some function $Z_{(m, n)}^{\left(v_{1}, v_{2}\right)}\left(\tau, a_{f}\right)$ whose physical meaning is unclear. However, we expect them to be related to the partition function with the inclusion of more general defects and leave a detailed analysis for the future.

${ }^{111}$ In fact, in Refs. 44 and 45 , this was confirmed explicitly. Here, we take a different approach, but we claim to obtain the relevant four-dimensional background on flat space $\mathbb{R}_{\varepsilon}^{2} \oplus \mathbb{R}^{2}$.

${ }^{112}$ In the following, we will take the $\varepsilon \rightarrow 0$ expansion, and thus, $\rho$ turns into the decompactified radial coordinate on $\mathbb{R}_{\varepsilon}^{2}$.

${ }^{113}$ L. F. Alday, M. Bullimore, and M. Fluder, “On S-duality of the superconformal index on lens spaces and 2d TQFT,” J. High Energy Phys. 2013(5), 122; arXiv:1301.7486 [hep-th].

${ }^{114}$ S. S. Razamat and M. Yamazaki, "S-duality and the N = 2 lens space index," J. High Energy Phys. 2013(10), 048; arXiv:1306.1543 [hep-th].

${ }^{115}$ S. S. Razamat and B. Willett, "Global properties of supersymmetric theories and the lens space," Commun. Math. Phys. 334, 661-696 (2015); arXiv:1307.4381.

${ }^{116}$ T. T. Dumitrescu and G. Festuccia, “In progress," Future 1 (2112).

${ }^{117}$ D. Gaiotto, “Twisted compactifications of 3d $\mathscr{N}=4$ theories and conformal blocks," J. High Energy Phys. 2019(2), 061; arXiv:1611.01528 [hep-th].

${ }^{118}$ C. Beem, L. Rastelli, and B. C. van Rees, “ $\mathscr{W}$ symmetry in six dimensions," J. High Energy Phys. 2015(5), 017; arXiv:1404.1079 [hep-th].

${ }^{119}$ S. M. Chester, J. Lee, S. S. Pufu, and R. Yacoby, “Exact correlators of BPS operators from the 3d superconformal bootstrap," J. High Energy Phys. 2015(3), 130; arXiv: 1412.0334 [hep-th].

${ }^{120}$ C. Beem, W. Peelaers, and L. Rastelli, “Deformation quantization and superconformal symmetry in three dimensions," Commun. Math. Phys. 354, 345-392 (2017); arXiv: 1601.05378 [hep-th].

${ }^{121}$ M. Mezei, S. S. Pufu, and Y. Wang, “Chern-Simons theory from M5-branes and calibrated M2-branes," J. High Energy Phys. 2019, 165; arXiv:1812.07572 [hep-th].

${ }^{122}$ M. Dedushenko, Y. Fan, S. S. Pufu, and R. Yacoby, “Coulomb branch quantization and abelianized monopole bubbling," J. High Energy Phys. 2019, 179; arXiv:1812.08788 [hep-th].

${ }^{123}$ M. Bullimore, T. Dimofte, and D. Gaiotto, “The Coulomb branch of 3d $\mathscr{N}=4$ theories," Commun. Math. Phys. 354, 671-751 (2017); arXiv:1503.04817 [hep-th].

${ }^{124}$ M. Bullimore, T. Dimofte, D. Gaiotto, and J. Hilburn, "Boundaries, mirror symmetry, and symplectic duality in $3 \mathrm{~d} \mathscr{N}=4$ gauge theory," J. High Energy Phys. 2016(10), 108; arXiv:1603.08382 [hep-th].

${ }^{125}$ M. Bullimore, T. Dimofte, D. Gaiotto, J. Hilburn, and H.-C. Kim, “Vortices and vermas,” Adv. Theor. Math. Phys. 22, $803-917$ (2018); arXiv:1609.04406 [hep-th].

${ }^{126}$ T. Dimofte and N. Garner, "Coulomb branches of star-shaped quivers," J. High Energy Phys. 2019(2), 004; arXiv:1808.05226 [hep-th].

${ }^{127}$ S. Cecotti, A. Neitzke, and C. Vafa, "R-twisting and 4d/2d correspondences," arXiv:1006.3435 [hep-th] (2010).

${ }^{128}$ L. Fredrickson and A. Neitzke, "From $S^{1}$-fixed points to $\mathscr{W}$-algebra representations," arXiv:1709.06142 [math.DG] (2017).

${ }^{129}$ M. Dedushenko, S. Gukov, H. Nakajima, D. Pei, and K. Ye, “3d TQFTs from Argyres-Douglas theories," arXiv:1809.04638 [hep-th] (2018).

${ }^{130}$ Throughout the text, we denote by $\tau$ the Pauli matrices and by $\sigma$ the $2 \times 2$ blocks inside the four-dimensional gamma matrices.

${ }^{131}$ For ease of notation, we will neglect the trace over the gauge indices here.

${ }^{132}$ Our conventions for the four-dimensional Hodge star operation is fixed as follows: for two $p$-forms $\alpha_{1}$ and $\alpha_{2}$, we have $\alpha_{1} \wedge * \alpha_{2}=\frac{1}{p !}\left(\alpha_{1}\right)_{m_{1} \cdots m_{p}}\left(\alpha_{2}\right)^{m_{1} \cdots m_{p}}$.

${ }^{133}$ Note that the derivatives of $F$ are vanishing due to the Bianchi identity.

${ }^{134}$ T. T. Dumitrescu, G. Festuccia, and N. Seiberg, “Exploring curved superspace," J. High Energy Phys. 2012(8), 141; arXiv:1205.1115 [hep-th].

${ }^{135}$ The ambiguous use of the letter $\zeta$ as the Killing spinors and the deformation of the metric (18) in the main text is unfortunate. However, it should be clear from the context which one is referred to. 
${ }^{136}$ Note that compared with Ref. 134, we have slightly differing conventions, which explains the appearance of some alternate normalization in the following.

${ }^{137}$ Note that although this choice of gamma matrices agrees with the ones in the main text, they do differ from the conventions in Ref. 134.

${ }^{138}$ Note that the spinors could be fixed to be periodic by absorbing an appropriate factor into the background fields. However, with an eye towards embedding the $\mathscr{N}=1$ background into the $\mathscr{N}=2$ one, we have chosen this (unusual) periodicity, which turns out to match with the twisted-sector requirement of the $\mathscr{N}=2$ spinors. ${ }^{139}$ Recall that half-hypermultiplets in a representation $\mathbf{R}=\mathscr{R} \oplus \overline{\mathscr{R}}$ are called full hypermultiplets.

${ }^{140}$ A. Gadde, L. Rastelli, S. S. Razamat, and W. Yan, “On the superconformal index of N = 1 IR fixed points: A holographic check,” J. High Energy Phys. 2011(3), 041; arXiv:1011.5278 [hep-th].

${ }^{141}$ K. Maruyoshi and J. Song, “ $\mathscr{N}=1$ deformations and RG flows of $\mathscr{N}=2$ SCFTs,” J. High Energy Phys. 2017(2), 075; arXiv:1607.04281 [hep-th].

${ }^{142}$ We stress again that we are dealing with a full hypermultiplet in a representation $\mathscr{R}$, which corresponds to half-hypermultiplets in $\mathbf{R}=\mathscr{R} \oplus \overline{\mathscr{R}}$.

${ }^{143}$ A. Gadde, E. Pomoni, L. Rastelli, and S. S. Razamat, "S-duality and 2d topological QFT," J. High Energy Phys. 2010(3), 032; arXiv:0910.2225 [hep-th].

${ }^{144}$ D. Gaiotto, L. Rastelli, and S. S. Razamat, "Bootstrapping the superconformal index with surface defects," J. High Energy Phys. 2013(1), 022; arXiv:1207.3577 [hep-th].

${ }^{145}$ In the first expression for the vector multiplet, we take product over all the roots of $G$, including the zero-roots, and in the latter, we rewrite it in terms of positive roots.

${ }^{146}$ A. Gadde and S. Gukov, “2d index and surface operators," J. High Energy Phys. 2014, 80; arXiv:1305.0266 [hep-th].

${ }^{147}$ K. Hosomichi, S. Lee, and T. Okuda, "Supersymmetric vortex defects in two dimensions," J. High Energy Phys. 2018(1), 033; arXiv:1705.10623 [hep-th].

${ }^{148}$ Note that on a technical level, our frame in (19) is ill-suited for this, as it does not smoothly continue through $\theta=0$. Thus, we performed this calculation in an alternative frame, closely related to the one in (C3).

${ }^{149}$ N. Drukker, T. Okuda, and F. Passerini, “Exact results for vortex loop operators in 3d supersymmetric theories,” J. High Energy Phys. 2014, 137 (2014).

${ }^{150}$ We use the shorthand matrix notation for $\xi=\left(\xi_{A \alpha}\right)$ and $\bar{\xi}=\left(\bar{\xi}_{A}{ }^{\dot{\alpha}}\right)$.

${ }^{151}$ A. Karlhede and M. Roček, “Topological quantum field theory and N = 2 conformal supergravity," Phys. Lett. B 212, 51-55 (1988).

${ }^{152}$ A. Galperin and O. Ogievetsky, "Holonomy groups, complex structures and D = 4 topological Yang-Mills theory," Commun. Math. Phys. 139, 377-394 (1991).

${ }^{153}$ A. Johansen, "Twisting of $N=1$ SUSY gauge theories and heterotic topological theories," Int. J. Mod. Phys. A 10, 4325-4358 (1995); arXiv:hep-th/9403017 [hep-th].

${ }^{154} \mathrm{D}$. Sen, "Extended supersymmetry in the space-time R $\times \mathrm{S}^{3}$," Phys. Rev. D 41, 667 (1990).

${ }^{155}$ C. Klare and A. Zaffaroni, "Extended supersymmetry on curved spaces," J. High Energy Phys. 2013(10), 218; arXiv:1308.1102 [hep-th].

${ }^{156}$ S. Gukov and E. Witten, "Gauge theory, ramification, and the geometric Langlands program," arXiv:hep-th/0612073 [hep-th] (2006).

${ }^{157}$ S. Gukov and E. Witten, "Rigid surface operators," Adv. Theor. Math. Phys. 14, 87 (2010); arXiv:0804.1561 [hep-th].

${ }^{158}$ A. Kapustin, B. Willett, and I. Yaakov, “Exact results for supersymmetric abelian vortex loops in 2+1 dimensions," J. High Energy Phys. 2013(6), 099; arXiv:1211.2861 [hep-th].

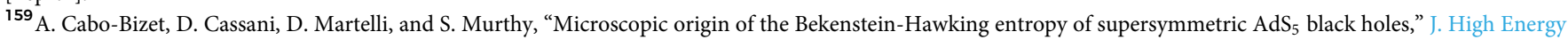
Phys. 2019, 62; arXiv:1810.11442 [hep-th]. 